\title{
Formation and Utility of Oxasilacyclopentenes from Functionalized Alkynes
}

\author{
Timothy B. Clark and K. A. Woerpel* \\ Department of Chemistry, University of California, \\ Irvine, California 92697-2025
}

\section{Contents:}

\section{Supporting Information}

I. $\quad \mathrm{Ag}_{3} \mathrm{PO}_{4}$-catalyzed Construction of Silacyclopropenes...................S-2

II. Characterization Data on Silacyclopropenes................................S-3

III. Construction and Utility of Oxasilacyclopentenes.........................S-5

IV. Regiochemistry Assignment of Oxasilacyclopentenes by NOE.............S-13

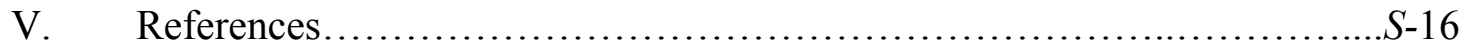

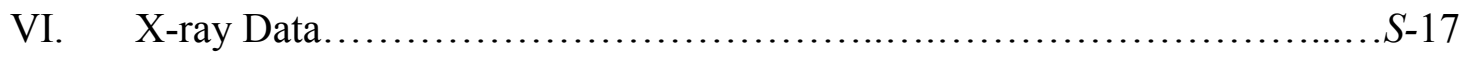

VII. Spectra of ${ }^{1} \mathrm{H}$ NMR Monitored Reactions ............................... 56

VIII. Selected Spectra......................................................... 64

\section{Experimental Section}

General. Melting points were obtained using a Büchi 510 melting point apparatus and were reported uncorrected. Analytical gas chromatography (GC) was performed on an Agilent 6850 series chromatograph, equipped with an Agilent 6850 auto sampler and a flame ionization detector (a fused silica capillary flash $(30 \mathrm{~m}$ x $0.32 \mathrm{~mm}$ x $0.25 \mu \mathrm{m}$ ) wall-coated with HP-1 (J \& W Scientific) was used with helium as the carrier gas) or on a gas chromatography-mass spectrometry (GC-MS) Thermo-Finnigan Trace Mass Spectrometer Plus quadrapole system with a fused silica capillary flash $(30 \mathrm{~m} \times 0.32 \mathrm{~mm} \times 0.25 \mu \mathrm{m})$ wall-coated with DB-5 (J \& W Scientific) using electron ionization $(70 \mathrm{eV})$. Analytical thin layer chromatography was performed on EM reagents $0.25 \mathrm{~mm}$ silica gel $60-\mathrm{F}$ plates. Liquid chromatography was performed using forced flow (flash chromatography) of the indicated solvent system on EM reagents silica gel $\left(\mathrm{SiO}_{2}\right) 60(230-400)$ mesh or on Aldrich Davasil ${ }^{8}$ silica gel $\left(\mathrm{SiO}_{2}\right)$ as indicated. ${ }^{1} \mathrm{H}$ NMR and ${ }^{13} \mathrm{C}$ NMR spectra were recorded at $25{ }^{\circ} \mathrm{C}$ at 400 and 100, and 500 and $125 \mathrm{MHz}$, respectively, using Bruker DRX 400 or DRX 500 spectrometers, as indicated. The data are reported as follows: chemical shift in ppm from internal tetramethylsilane on the $\delta$ scale, multiplicity ( $\mathrm{br}=$ broad, $\mathrm{s}=$ singlet, $\mathrm{d}=$ doublet, $\mathrm{t}=$ triplet, $\mathrm{q}=$ quartet, $\mathrm{p}=$ pentet, $\mathrm{h}=$ hextet, $\mathrm{m}=$ multiplet), coupling constants $(\mathrm{Hz})$, and integration. The ${ }^{29} \mathrm{Si}$ NMR chemical shifts are reported in ppm from tetramethylsilane on the $\delta$ scale, using tetramethylsilane as an external standard. High resolution mass spectra were acquired on a VG Analytical 7070E or Fisons Autospec spectrometer, and were obtained by peak matching. Microanalyses were performed by Atlantic Microlabs, Atlanta, GA or Desert Analytics, Tucson, AZ. Silacyclopropanes and silacyclopropenes were stored and manipulated in an Innovative Technologies nitrogen atmosphere dry box. All reaction mixtures to form silacyclopropenes and oxasilacyclopentenes unless specified were prepared in the dry box. All other reactions unless specified were carried out under an atmosphere of nitrogen or Argon in glassware that had been flame-dried under a stream of nitrogen or under vacuum. Solvents were distilled or filtered before use. 4-triisopropylsilyloxy-2-butyne ${ }^{1}$ (Table 1, entry 6) and cyclohexenesilacyclopropane ${ }^{2,3}(\mathbf{1})$ were constructed by known methods. 


\section{I. $\quad \mathrm{Ag}_{3} \mathrm{PO}_{4}$-catalyzed Construction of Silacyclopropenes}

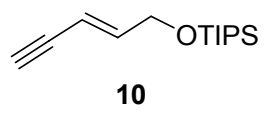

5-(triisopropylsilyloxy)-3-penten-1-yne (10).

To a cooled $\left(0^{\circ} \mathrm{C}\right)$ solution of 3-penten-5-yn-1-ol (3.96 g, $\left.46.3 \mathrm{mmol}\right)$, imidazole $(6.30 \mathrm{~g}, 92.6 \mathrm{mmol})$ and 12.0 $\mathrm{mL}$ of $\mathrm{N}, \mathrm{N}$-dimethylformamide was added triisopropylsilylchloride $(10.4 \mathrm{~mL}, 48.6 \mathrm{mmol})$. The reaction mixture was allowed to warm to room temperature and stir for $21 \mathrm{~h}$. The reaction mixture was diluted with 50 $\mathrm{mL}$ of water and extracted with $\mathrm{Et}_{2} \mathrm{O}(3 \mathrm{x} 40 \mathrm{~mL})$. The organic extracts were rinsed with $1.0 \mathrm{~N}$ aqueous $\mathrm{HCl}(2$ x $30 \mathrm{~mL})$, saturated aqueous $\mathrm{NaCl}(30 \mathrm{~mL})$, dried with $\mathrm{MgSO}_{4}$ and concentrated in vacuo to give a yellow oil. Purification by column chromatography (hexanes to $3: 97 \mathrm{CH}_{2} \mathrm{Cl}_{2}$ :hexanes) afforded $\mathbf{1 0}$ as a colorless oil (6.80 g, 72\%): ${ }^{1} \mathrm{H} \mathrm{NMR}\left(\mathrm{CDCl}_{3}, 500 \mathrm{MHz}\right) \delta 6.31(\mathrm{dt}, J=15.8,3.9,1 \mathrm{H}), 5.82(\mathrm{dq}, J=15.8,2.3,1 \mathrm{H}), 4.33(\mathrm{t}, J=3.0$, $2 \mathrm{H}), 2.87(\mathrm{~d}, J=2.2,1 \mathrm{H}), 1.07(\mathrm{~m}, 21 \mathrm{H}) ;{ }^{13} \mathrm{C} \mathrm{NMR}\left(\mathrm{CDCl}_{3}, 125 \mathrm{MHz}\right) \delta 144.6,107.6,82.4,77.5,63.1,18.2$, 12.2; IR (thin film) 3315. 2944, 2867, 1464, $882 \mathrm{~cm}^{-1}$; HRMS: $\left(\mathrm{NH}_{3} \mathrm{CI}\right) \mathrm{m} / \mathrm{z}$ calcd for $\mathrm{C}_{14} \mathrm{H}_{25} \mathrm{OSi}(\mathrm{M}-\mathrm{H})^{7}$ 237.1675, found 237.1674. Anal. Calcd for $\mathrm{C}_{14} \mathrm{H}_{26} \mathrm{OSi}$ : C, 70.52; H, 10.99. Found: C, 70.25; H, 11.17.

$\mathrm{Ag}_{3} \mathrm{PO}_{4}$-catalyzed silacyclopropenation of alkynes typically utilized a $1: 1$ ratio of alkyne:cyclohexenesilacyclopropene 1. In the following two examples, a small excess of 1 was used for increased yields. For substrate ratios, see experimental procedures for the in situ formation of oxasilacyclopentenes from alkynes.

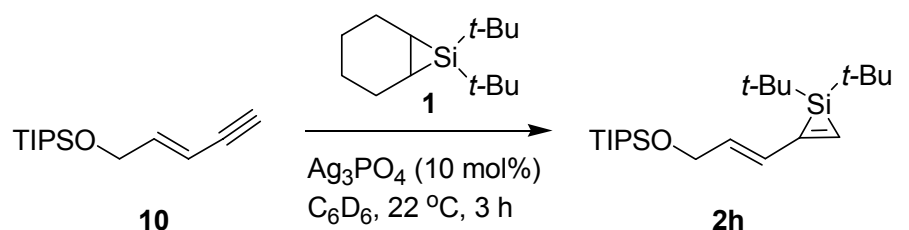

1,1-Di-tert-butyl-2-(3-triisopropylsilyloxy-1-propenyl)silacyclopropene $(2 \mathrm{~h})$. Representative Procedure for the silver-catalyzed silacyclopropenation of synthesized alkynes:

To an NMR tube containing $\mathrm{Ag}_{3} \mathrm{PO}_{4}(0.005 \mathrm{~g}, 0.012 \mathrm{mmol})$ was added the alkyne $(0.230 \mathrm{~mL}, 0.115 \mathrm{mmol}$, $0.500 \mathrm{M}$ solution of alkyne and $0.133 \mathrm{M}$ solution of $\mathrm{PhSiMe}_{3}$, the internal standard in $\mathrm{C}_{6} \mathrm{D}_{6}$ ), followed by 1 $\left(0.270 \mathrm{~mL}, 0.135 \mathrm{mmol}, 0.500 \mathrm{M}\right.$ solution of 1 and $0.133 \mathrm{M}$ solution of $\mathrm{PhSiMe}_{3}$ in $\left.\mathrm{C}_{6} \mathrm{D}_{6}\right)$. The reaction yield was determined to be $81 \%$ by ${ }^{1} \mathrm{H}$ NMR spectroscopy (compared to the $\mathrm{PhSiMe}_{3}$ internal standard) using a single scan: ${ }^{1} \mathrm{H}$ NMR $\left(\mathrm{C}_{6} \mathrm{D}_{6}, 500 \mathrm{MHz}\right) \delta 7.91(\mathrm{~s}, 1 \mathrm{H}), 6.81(\mathrm{dt}, J=14.9,1.7,1 \mathrm{H}), 6.11(\mathrm{dt}, J=14.8,4.7,1 \mathrm{H})$, $4.29(\mathrm{ddd}, J=4.7,1.9,0.5,2 \mathrm{H}), 1.10(\mathrm{~s}, 39 \mathrm{H}) ;{ }^{13} \mathrm{C}$ NMR $\left(\mathrm{C}_{6} \mathrm{D}_{6}, 125 \mathrm{MHz}\right) \delta 160.3,144.7,140.3,125.1,64.2$, 30.5, 21.4, 18.6, 12.8; ${ }^{29} \mathrm{Si}$ NMR $\left(\mathrm{C}_{6} \mathrm{D}_{6}, 99.3 \mathrm{MHz}\right)-67.5,13.5$.

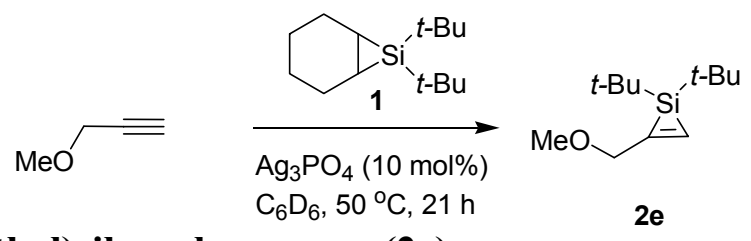

1,1-Di-tert-butyl-2-(methoxymethyl)silacyclopropene (2e).

Representative Procedure for the silver-catalyzed silacyclopropenation of commercially available alkynes:

To an NMR tube containing $\mathrm{Ag}_{3} \mathrm{PO}_{4}(0.006 \mathrm{~g}, 0.014 \mathrm{mmol})$ was added the alkyne $(0.012 \mathrm{~mL}, 0.142 \mathrm{mmol})$, followed by $1\left(0.600 \mathrm{~mL}, 0.150 \mathrm{mmol}, 0.250 \mathrm{M}\right.$ solution of 1 and $0.133 \mathrm{M}$ solution of $\left.\mathrm{PhSiMe}_{3} \mathrm{in}_{6} \mathrm{D}_{6}\right)$. $\mathrm{The}$ reaction yield was determined to be $79 \%$ by ${ }^{1} \mathrm{H}$ NMR spectroscopy (compared to the $\mathrm{PhSiMe}_{3}$ internal standard) using a single scan: ${ }^{1} \mathrm{H}$ NMR $\left(\mathrm{C}_{6} \mathrm{D}_{6}, 500 \mathrm{MHz}\right) \delta 8.05(\mathrm{t}, J=1.9,1 \mathrm{H}), 4.34(\mathrm{~d}, J=1.9,1 \mathrm{H}), 3.26(\mathrm{~s}, 3 \mathrm{H}), 1.05(\mathrm{~s}$, $18 \mathrm{H}) ;{ }^{13} \mathrm{C}$ NMR $\left(\mathrm{C}_{6} \mathrm{D}_{6}, 125 \mathrm{MHz}\right) \delta 162.9,138.1,73.7,58.2,30.3,21.4 ;{ }^{29} \mathrm{Si} \mathrm{NMR}\left(\mathrm{C}_{6} \mathrm{D}_{6}, 99.3 \mathrm{MHz}\right)-66.6$. 


\section{Characterization Data on Silacyclopropenes}

\section{Characterization Data for Silacyclopropenes by Thermal Silacyclopropenation}

Isolation of silacyclopropenes generated by the silver-catalyzed silacyclopropenation was challenging, so, several of the reported silacyclopropenes were constructed by thermal silacyclopropenation and characterized. Thermal silacyclopropenation was found to provide increased yields with a sealed vessel under static vacuum (as described ${ }^{4}$ ). For internal alkynes, no solvent was utilized; terminal alkynes provided fewer by-products using toluene as solvent.

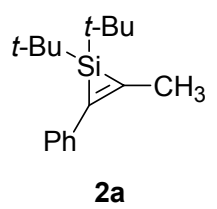

1,1-Di-tert-butyl-2-phenyl-3-methylsilacyclopropene (2a).

Representative Procedure for the thermal silacyclopropenation of alkynes:

A mixture of $1(1.74 \mathrm{~g}, 7.75 \mathrm{mmol})$ and 1-phenyl-1-propyne $(0.900 \mathrm{~g}, 7.75 \mathrm{mmol})$ was added to a vessel fitted with an air-free seal. The reaction mixture was degassed. The reaction mixture was heated to $90{ }^{\circ} \mathrm{C}$ for $72 \mathrm{~h}$. After cooling to $22{ }^{\circ} \mathrm{C}$, the reaction mixture was concentrated in vacuo. Purification by bulb-to-bulb distillation $\left(90-100{ }^{\circ} \mathrm{C} / 0.5 \mathrm{~mm} \mathrm{Hg}\right)$ gave $2 \mathrm{a}$ as a colorless liquid $(1.57 \mathrm{~g}, 53 \%):{ }^{1} \mathrm{H}$ NMR $\left(\mathrm{C}_{6} \mathrm{D}_{6}, 500 \mathrm{MHz}\right) \delta 7.53(\mathrm{dd}, J=$ $8.0,1.2,2 \mathrm{H}), 7.25(\mathrm{t}, J=7.7,2 \mathrm{H}), 7.09(\mathrm{~m}, 1 \mathrm{H}), 2.34(\mathrm{~s}, 3 \mathrm{H}), 1.11(\mathrm{~s}, 18 \mathrm{H}) ;{ }^{13} \mathrm{C}$ NMR $\left(\mathrm{C}_{6} \mathrm{D}_{6}, 125 \mathrm{MHz}\right)$ $\delta 152.6,151.7,137.9,131.6,130.5,128.9,32.0,23.4,17.5 ;{ }^{29} \mathrm{Si}$ NMR $\left(\mathrm{C}_{6} \mathrm{D}_{6}, 99.3 \mathrm{MHz}\right)-68.4$; IR (thin film) 3075, 2929, 2856, 1620, 1470, 822, $760 \mathrm{~cm}^{-1}$; HRMS (EI/GCMS) $\mathrm{m} / z$ calcd for $\mathrm{C}_{17} \mathrm{H}_{26} \mathrm{Si}(\mathrm{M})^{+} 258.1804$, found 258.1805. Anal. Calcd for $\mathrm{C}_{17} \mathrm{H}_{26} \mathrm{Si}$ : C, 79.00; H, 10.14. Found: C, 78.63; H, 9.99.

\section{1,1-Di-tert-butyl-2,3-diethylsilacyclopropene (2b). ${ }^{5}$}

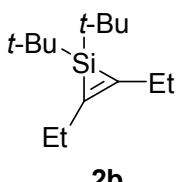

The representative procedure for thermal silacyclopropenation of alkynes was followed using 1 (3.00 g, 13.4 $\mathrm{mmol})$ and 3-hexyne $(1.10 \mathrm{~g}, 13.4 \mathrm{mmol})$ at $90{ }^{\circ} \mathrm{C}$ for $48 \mathrm{~h}$. Purification by bulb-to-bulb distillation $(30-40$ ${ }^{\circ} \mathrm{C} / 0.5 \mathrm{~mm} \mathrm{Hg}$ ) gave $2 \mathbf{b}$ as a colorless liquid $(2.42 \mathrm{~g}, 81 \%)$ (spectral data matched that reported $\left.{ }^{5}\right):{ }^{1} \mathrm{H}$ NMR $\left(\mathrm{C}_{6} \mathrm{D}_{6}, 500 \mathrm{MHz}\right) \delta 2.45(\mathrm{q}, J=8.5,4 \mathrm{H}), 1.14(\mathrm{t}, J=8.5,6 \mathrm{H}), 1.11(\mathrm{~s}, 18 \mathrm{H}) ;{ }^{13} \mathrm{C}$ NMR $\left(\mathrm{C}_{6} \mathrm{D}_{6}, 125 \mathrm{MHz}\right)$ $\delta$ 152.9, 30.6, 22.6, 21.3, 15.3; ${ }^{29} \mathrm{Si}$ NMR $\left(\mathrm{C}_{6} \mathrm{D}_{6}, 99.3 \mathrm{MHz}\right)-66.8$; IR (thin film) 2942, 2961, 2855, 1470, 821 $\mathrm{cm}^{-1}$; HRMS (EI/GCMS) $\mathrm{m} / z$ calcd for $\mathrm{C}_{14} \mathrm{H}_{28} \mathrm{Si}(\mathrm{M})^{+} 224.1960$, found 224.1959. Anal. Calcd for $\mathrm{C}_{14} \mathrm{H}_{28} \mathrm{Si}: \mathrm{C}$, 74.91; H, 12.57. Found: C, 74.63; H, 12.53 .

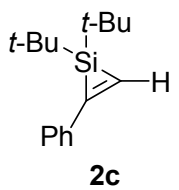

1,1-Di-tert-butyl-2-phenylsilacyclopropene (2c).

The representative procedure for thermal silacyclopropenation of alkynes was followed using 1 (3.30 g, 14.7 $\mathrm{mmol}$ ), phenylacetylene $(1.50 \mathrm{~g}, 14.7 \mathrm{mmol})$, and $15.0 \mathrm{~mL}$ of toluene at $90{ }^{\circ} \mathrm{C}$ for $72 \mathrm{~h}$. Purification by bulb-tobulb distillation $\left(60-70{ }^{\circ} \mathrm{C} / 0.5 \mathrm{~mm} \mathrm{Hg}\right)$ gave $2 \mathrm{c}$ as a colorless liquid $(3.38 \mathrm{~g}, 94 \%):{ }^{1} \mathrm{H}$ NMR $\left(\mathrm{C}_{6} \mathrm{D}_{6}, 500 \mathrm{MHz}\right)$ $\delta 8.31(\mathrm{~s}, 1 \mathrm{H}), 7.57(\mathrm{dd}, J=8.2,1.3,2 \mathrm{H}), 7.23(\mathrm{t}, J=8.2,2 \mathrm{H}), 7.11(\mathrm{~m}, 1 \mathrm{H}), 1.10(\mathrm{~s}, 18 \mathrm{H}) ;{ }^{13} \mathrm{C}$ NMR $\left(\mathrm{C}_{6} \mathrm{D}_{6}\right.$, 
$125 \mathrm{MHz}) \delta 162.2,139.2,135.4,129.6,129.2,128.9,30.5,21.5 ;{ }^{29} \mathrm{Si} \mathrm{NMR}\left(\mathrm{C}_{6} \mathrm{D}_{6}, 99.3 \mathrm{MHz}\right)-65.2 ;$ IR (thin film) $3021,2929,2856,1470,823,744 \mathrm{~cm}^{-1}$; HRMS (EI/GCMS) $m / z$ calcd for $\mathrm{C}_{16} \mathrm{H}_{24} \mathrm{Si}(\mathrm{M})^{+} 244.1647$, found 244.1644. Anal. Calcd for $\mathrm{C}_{16} \mathrm{H}_{24} \mathrm{Si}$ : C, 78.61; H, 9.90. Found: C, 78.57; H, 9.84.

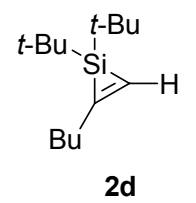

\section{1,1-Di-tert-butyl-2-butylsilacyclopropene (2d).}

The representative procedure for thermal silacyclopropenation of alkynes was followed using $\mathbf{1}$ (2.19 g, 9.70 mmol), 1-hexyne $(3.2 \mathrm{~g}, 39.0 \mathrm{mmol})$, and $5.0 \mathrm{~mL}$ of toluene at $90{ }^{\circ} \mathrm{C}$ for $48 \mathrm{~h}$. Purification by bulb-to-bulb distillation $\left(30-40{ }^{\circ} \mathrm{C} / 0.5 \mathrm{~mm} \mathrm{Hg}\right)$ gave $2 \mathrm{~d}$ as a colorless liquid $(1.75 \mathrm{~g}, 80 \%)$ in a 96:4 mixture of $2 \mathrm{~d}: \mathbf{1}:{ }^{1} \mathrm{H}$ $\operatorname{NMR}\left(\mathrm{C}_{6} \mathrm{D}_{6}, 500 \mathrm{MHz}\right) \delta 7.85(\mathrm{t}, J=1.5,1 \mathrm{H}), 2.51(\mathrm{dt}, J=7.4,1.5,2 \mathrm{H}), 1.58(\mathrm{~m}, 2 \mathrm{H}), 1.39(\mathrm{~h}, J=7.5,2 \mathrm{H})$, $1.09(\mathrm{~s}, 18 \mathrm{H}), 0.92(\mathrm{t}, J=7.3,3 \mathrm{H}) ;{ }^{13} \mathrm{C} \mathrm{NMR}\left(\mathrm{C}_{6} \mathrm{D}_{6}, 125 \mathrm{MHz}\right) \delta 166.3,137.8,33.4,31.8,30.6,23.3,21.3$, 14.6; ${ }^{29} \mathrm{Si}$ NMR $\left(\mathrm{C}_{6} \mathrm{D}_{6}, 99.3 \mathrm{MHz}\right)-66.7$; IR (thin film) 2959, 2856, 1559, 1470,823 $\mathrm{cm}^{-1}$; HRMS (EI/GCMS) $m / z$ calcd for $\mathrm{C}_{14} \mathrm{H}_{28} \mathrm{Si}(\mathrm{M})^{+} 224.1960$, found 224.1960 .

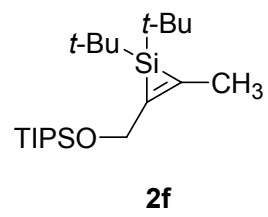

\section{1,1-Di-tert-butyl-2-(triisopropylsilyloxymethyl)-3-methylsilacyclopropene (2f).}

The representative procedure for thermal silacyclopropenation of alkynes was followed using 1 (0.660 g, 2.94 $\mathrm{mmol})$ and triisopropylsilyoxy-2-butyne $(0.665 \mathrm{~g}, 2.94 \mathrm{mmol})$ at $90{ }^{\circ} \mathrm{C}$ for $4 \mathrm{~d}$. Purification by bulb-to-bulb distillation $\left(140-150{ }^{\circ} \mathrm{C} / 0.5 \mathrm{~mm} \mathrm{Hg}\right)$ gave $2 \mathrm{f}$ as a colorless liquid $(0.525 \mathrm{~g}, 53 \%):{ }_{13}^{1} \mathrm{H} \mathrm{NMR}\left(\mathrm{C}_{6} \mathrm{D}_{6}, 500 \mathrm{MHz}\right) \delta$ $4.81(\mathrm{q}, J=1.5,2 \mathrm{H}), 2.32(\mathrm{t}, J=1.5,3 \mathrm{H}), 1.12(\mathrm{~m}, 21 \mathrm{H}), 1.08(\mathrm{~s}, 18 \mathrm{H}) ;{ }^{13} \mathrm{C} \mathrm{NMR}\left(\mathrm{C}_{6} \mathrm{D}_{6}, 125 \mathrm{MHz}\right)$ $\delta$ 151.2, 147.4, 64.4, 30.3, 21.7, 18.4, 15.8, 12.4; ${ }^{29} \mathrm{Si}$ NMR $\left(\mathrm{C}_{6} \mathrm{D}_{6}, 99.3 \mathrm{MHz}\right)-65.4,12.6$; IR (thin film) 2942, 2866, 1646, 1470, 1085, 1052, $821 \mathrm{~cm}^{-1}$; HRMS (EI/GCMS) $m / z$ calcd for $\mathrm{C}_{18} \mathrm{H}_{37} \mathrm{OSi}_{2}\left[\mathrm{M}-\mathrm{C}_{3} \mathrm{H}_{7}\right]^{+} 325.2383$, found 325.2372. Anal. Calcd for $\mathrm{C}_{21} \mathrm{H}_{44} \mathrm{OSi}_{2}$ : C, 68.40; H, 12.03. Found: C, 68.57; H, 12.04 .

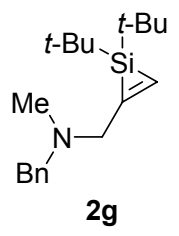

\section{$N$-Benzyl- $N$-(1,1-Di-tert-butyl-2-silacyclopropenyl)-methylamine (2g).}

The representative procedure for thermal silacyclopropenation of alkynes was followed using $\mathbf{1}$ (2.96 g, 13.2 mmol), $N$-methyl- $N$-propargylbenzylamine $(2.03 \mathrm{~mL}, 12.0 \mathrm{mmol})$ and toluene $(30.0 \mathrm{~mL})$ at $90-100{ }^{\circ} \mathrm{C}$ for $7 \mathrm{~d}$. Purification by bulb-to-bulb distillation $\left(110-130{ }^{\circ} \mathrm{C} / 0.5 \mathrm{~mm} \mathrm{Hg}\right)$ gave $2 \mathrm{~g}$ as a pale yellow liquid $(1.19 \mathrm{~g}, 34 \%)$ : ${ }^{1} \mathrm{H}$ NMR $\left(\mathrm{C}_{6} \mathrm{D}_{6}, 500 \mathrm{MHz}\right) \delta 8.01(\mathrm{t}, J=1.5,1 \mathrm{H}), 7.45(\mathrm{~d}, J=7.7,2 \mathrm{H}), 7.21(\mathrm{t}, J=7.6,2 \mathrm{H}), 7.11(\mathrm{t}, J=7.4$, $1 \mathrm{H}), 3.58(\mathrm{~s}, 2 \mathrm{H}), 3.51(\mathrm{~s}, 2 \mathrm{H}), 2.28(\mathrm{~s}, 3 \mathrm{H}), 1.07(\mathrm{~s}, 18 \mathrm{H}) ;{ }^{13} \mathrm{C} \mathrm{NMR}\left(\mathrm{C}_{6} \mathrm{D}_{6}, 125 \mathrm{MHz}\right) \delta 163.5,140.7,139.1$, 129.4, 128.9, 127.5, 62.4, 60.3, 43.0, 30.5, 21.5; ${ }^{29} \mathrm{Si} \mathrm{NMR}\left(\mathrm{C}_{6} \mathrm{D}_{6}, 99.3 \mathrm{MHz}\right)-67.7$; IR (thin film) 2933, 2856, 1646, 1471, $823 \mathrm{~cm}^{-1}$; HRMS (EI/GCMS) $\mathrm{m} / \mathrm{z}$ calcd for $\mathrm{C}_{19} \mathrm{H}_{34} \mathrm{NOSi}\left[\mathrm{M}+\mathrm{H}_{3} \mathrm{O}\right]^{+} 320.2410$, found 320.2415 . 


\section{Construction and Utility of Oxasilacyclopentenes}

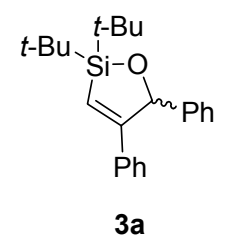

\section{2,2-Di-tert-butyl-4-phenyl-5-phenyloxasilacyclopentene (3a).}

Representative procedure for the two-step, one-flask formation of oxasilacyclopentenes:

To a mixture of $\mathrm{Ag}_{3} \mathrm{PO}_{4}(0.042 \mathrm{~g}, 0.100 \mathrm{mmol})$ and toluene $(4.0 \mathrm{~mL})$ was added phenylacetylene $(0.110 \mathrm{~mL}$, $1.00 \mathrm{mmol})$, followed by $1(0.225 \mathrm{~g}, 1.00 \mathrm{mmol})$. After 3 hours, the reaction mixture was cooled to $-20{ }^{\circ} \mathrm{C}$. Benzaldehyde $(0.305 \mathrm{~mL}, 3.00 \mathrm{mmol})$ was added, followed by $\mathrm{CuBr}_{2}(0.033 \mathrm{~g}, 0.148 \mathrm{mmol})$. The reaction mixture was allowed to warm to $22{ }^{\circ} \mathrm{C}$ over 1 hour. The reaction mixture was filtered through a short column of Davasil ${ }^{\circledR}$ with hexanes and concentrated in vacuo to give a colorless oil as a $\geq 99: 1$ mixture of regioisomers (as determined by gas chromatography). Purification by column chromatography (12:88 to $18: 82 \mathrm{CH}_{2} \mathrm{Cl}_{2}$ :hexanes) gave $18(0.276 \mathrm{~g}, 79 \%)$ as a colorless oil: $\mathrm{GC} t_{\mathrm{R}}=20.14\left(50-290{ }^{\circ} \mathrm{C}, 10{ }^{\circ} \mathrm{C} / \mathrm{min}\right) ;{ }^{1} \mathrm{H} \mathrm{NMR}\left(\mathrm{CDCl}_{3}, 500 \mathrm{MHz}\right)$ $\delta 7.29(\mathrm{~m}, 4 \mathrm{H}), 7.19(\mathrm{~m}, 6 \mathrm{H}), 6.57(\mathrm{~d}, J=2.0,1 \mathrm{H}), 6.12(\mathrm{~d}, J=2.0,1 \mathrm{H}), 1.07(\mathrm{~s}, 9 \mathrm{H}), 1.05(\mathrm{~s}, 9 \mathrm{H}) ;{ }^{13} \mathrm{C}$ NMR $\left(\mathrm{CDCl}_{3}, 125 \mathrm{MHz}\right) \delta 162.2,141.7,137.4,128.8,128.5,128.4,128.0,127.9,126.8,123.7,86.3,28.3,27.7,22.2$, 20.6; IR (thin film) 2931, 2856, 1557, 1472, 1037, $823 \mathrm{~cm}^{-1}$; HRMS $\left(\mathrm{NH}_{3} \mathrm{CI}\right) \mathrm{m} / z$ calcd for $\mathrm{C}_{23} \mathrm{H}_{30} \mathrm{OSi}(\mathrm{M})^{+}$ 350.2066, found 350.2064. Anal. Calcd for $\mathrm{C}_{23} \mathrm{H}_{30} \mathrm{OSi}$ : C, 78.80; H, 8.63. Found: C, 78.52; H, 8.77.

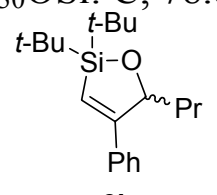

$3 \mathbf{b}$

\section{2,2-Di-tert-butyl-4-phenyl-5-propyloxasilacyclopentene (3b).}

The representative procedure for the two-step, one flask formation of oxasilacyclopentenes was followed with phenylacetylene $(0.147 \mathrm{~mL}, 1.34 \mathrm{mmol}), 1(0.300 \mathrm{~g}, 1.34 \mathrm{mmol})$ and $\mathrm{Ag}_{3} \mathrm{PO}_{4}(0.056 \mathrm{~g}, 0.134 \mathrm{mmol})$ for 3 hours and butyraldehyde $(0.362 \mathrm{~mL}, 4.02 \mathrm{mmol})$ and $\mathrm{CuBr}_{2}(0.045 \mathrm{~g}, 0.201 \mathrm{mmol})$ for 12 hours to provide a 99:1 unpurified mixture of regioisomers. Purification by column chromatography $\left(12: 88 \mathrm{CH}_{2} \mathrm{Cl}_{2}\right.$ :hexanes $)$ gave $\mathbf{3 b}$ $(0.335 \mathrm{~g}, 79 \%)$ as a colorless oil: $\mathrm{GC} \underline{t}_{\mathrm{R}}=16.55\left(50-290{ }^{\circ} \mathrm{C}, 10{ }^{\circ} \mathrm{C} / \mathrm{min}\right) ;{ }^{1} \mathrm{H} \mathrm{NMR}\left(\mathrm{CDCl}_{3}, 500 \mathrm{MHz}\right) \delta 7.35$ $(\mathrm{m}, 4 \mathrm{H}), 7.29(\mathrm{~m}, 1 \mathrm{H}), 6.10(\mathrm{~d}, J=1.8,1 \mathrm{H}), 5.13(\mathrm{dt}, J=9.9,2.0,1 \mathrm{H}), 1.62(\mathrm{~m}, 2 \mathrm{H}), 1.48(\mathrm{~m}, 1 \mathrm{H}), 1.29(\mathrm{q}, J=$ 9.9, $1 \mathrm{H}), 1.07(\mathrm{~s}, 9 \mathrm{H}), 1.01(\mathrm{~s}, 9 \mathrm{H}), 0.89(\mathrm{t}, J=7.2,3 \mathrm{H}) ;{ }^{13} \mathrm{C} \mathrm{NMR}\left(\mathrm{CDCl}_{3}, 125 \mathrm{MHz}\right) \delta 165.4,138.8,128.6$, 128.0, 126.6, 121.2, 83.7, 38.8, 28.2, 27.6, 21.7, 20.2, 19.8, 14.3; IR (thin film) 2931, 2857, 1556, 1472, 823, $754 \mathrm{~cm}^{-1}$; HRMS $\left(\mathrm{NH}_{3} \mathrm{CI}\right) \mathrm{m} / \mathrm{z}$ calcd for $\mathrm{C}_{20} \mathrm{H}_{32} \mathrm{OSi}(\mathrm{M})^{+} 316.2222$, found 316.2211. Anal. Calcd for $\mathrm{C}_{20} \mathrm{H}_{32} \mathrm{OSi}$ : C, 75.88; H, 10.19. Found: C, 75.72; H, 10.12.

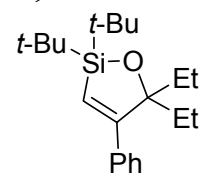

$3 c$

\section{2,2-Di-tert-butyl-4-phenyl-5,5-diethyloxasilacyclopentene (3c).}

The representative procedure for the two-step, one flask formation of oxasilacyclopentenes was followed with phenylacetylene $(0.110 \mathrm{~mL}, 1.00 \mathrm{mmol}), 1(0.225 \mathrm{~g}, 1.00 \mathrm{mmol})$ and $\mathrm{Ag}_{3} \mathrm{PO}_{4}(0.042 \mathrm{~g}, 0.100 \mathrm{mmol})$ for 3 hours and 3-pentanone $(0.318 \mathrm{~mL}, 3.00 \mathrm{mmol})$ and $\mathrm{CuBr}_{2}(0.033 \mathrm{~g}, 0.148 \mathrm{mmol})$ for 1 hour to provide a $\geq 99: 1$ unpurified mixture of regioisomers. Purification by column chromatography (hexanes to 1:99 $\mathrm{CH}_{2} \mathrm{Cl}_{2}$ :hexanes) gave 3c $(0.282 \mathrm{~g}, 85 \%)$ as a colorless oil: $\mathrm{GC} \underline{t}_{\mathrm{R}}=10.46\left(100-250{ }^{\circ} \mathrm{C}, 10^{\circ} \mathrm{C} / \mathrm{min}\right) ;{ }_{i}^{1} \mathrm{H} \mathrm{NMR}\left(\mathrm{CDCl}_{3}, 500 \mathrm{MHz}^{\circ}\right.$ $\delta 7.27(\mathrm{~m}, 5 \mathrm{H}), 5.70(\mathrm{~s}, 1 \mathrm{H}), 1.77(\mathrm{~m}, 4 \mathrm{H}), 1.07(\mathrm{~s}, 18 \mathrm{H}), 0.84(\mathrm{t}, J=7.5,6 \mathrm{H}) ;{ }^{13} \mathrm{C} \mathrm{NMR}\left(\mathrm{CDCl}_{3}, 125 \mathrm{MHz}^{2}\right.$ $\delta 171.7,142.4,128.0,127.9,127.2,122.9,90.3,31.7,28.6,20.7,9.4$; IR (thin film) 2932, 2856, 1563, 1472 , 977, $821 \mathrm{~cm}^{-1}$; HRMS $\left(\mathrm{NH}_{3} \mathrm{CI}\right) \mathrm{m} / \mathrm{z}$ calcd for $\mathrm{C}_{21} \mathrm{H}_{34} \mathrm{OSi}(\mathrm{M})^{+} 330.2379$, found 330.2381. Anal. Calcd for $\mathrm{C}_{21} \mathrm{H}_{34} \mathrm{OSi}$ : C, 76.30; H, 10.37. Found: C, 76.23; H, 10.49. 


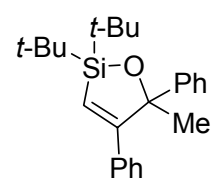

3d

2,2-Di-tert-butyl-4-phenyl-5-phenyl-5-methyloxasilacyclopentene (3d).

The representative procedure for the two-step, one flask formation of oxasilacyclopentenes was followed with phenylacetylene $(0.660 \mathrm{~mL}, 6.01 \mathrm{mmol}), 1(1.35 \mathrm{~g}, 6.01 \mathrm{mmol})$ and $\mathrm{Ag}_{3} \mathrm{PO}_{4}(0.251 \mathrm{~g}, 0.600 \mathrm{mmol})$ for 3 hours and acetophenone $(2.10 \mathrm{~mL}, 18.0 \mathrm{mmol})$ and $\mathrm{CuBr}_{2}(0.201 \mathrm{~g}, 0.900 \mathrm{mmol})$ for 2.5 hour to provide a $>95: 5$ unpurified mixture of regioisomers (as determined by ${ }^{1} \mathrm{H}$ NMR spectroscopy). Purification by column chromatography $\left(15: 85 \mathrm{CH}_{2} \mathrm{Cl}_{2}\right.$ :hexanes) gave 3d $(1.83 \mathrm{~g}, 83 \%)$ as a white solid: $\mathrm{mp}=78-79{ }^{\circ} \mathrm{C}$; $\mathrm{GC} \underline{t}_{\mathrm{R}}=$ $20.56\left(50-290{ }^{\circ} \mathrm{C}, 10{ }^{\circ} \mathrm{C} / \mathrm{min}\right) ;{ }^{1} \mathrm{H}$ NMR $\left(\mathrm{CDCl}_{3}, 500 \mathrm{MHz}\right) \delta 7.44(\mathrm{~m}, 2 \mathrm{H}), 7.30(\mathrm{~m}, 2 \mathrm{H}), 7.23(\mathrm{~m}, 6 \mathrm{H}), 6.44$ $(\mathrm{s}, 1 \mathrm{H}), 1.88(\mathrm{~s}, 3 \mathrm{H}), 1.10(\mathrm{~s}, 9 \mathrm{H}), 0.91(\mathrm{~s}, 9 \mathrm{H}) ;{ }^{13} \mathrm{C} \mathrm{NMR}\left(\mathrm{CDCl}_{3}, 125 \mathrm{MHz}\right) \delta 166.7,145.9,138.6,128.5$, 128.1, 127.9, 127.8, 127.4, 127.2, 124.3, 88.9, 29.2, 28.6, 28.6, 21.0, 20.8; IR (thin film) 3058, 2931, 2857, 1543, 1473, 951, $823 \mathrm{~cm}^{-1}$; HRMS $\left(\mathrm{NH}_{3} \mathrm{CI}\right) \mathrm{m} / \mathrm{z}$ calcd for $\mathrm{C}_{24} \mathrm{H}_{32} \mathrm{OSi}(\mathrm{M})^{+}$364.2222, found 364.2227. Anal. Calcd for $\mathrm{C}_{24} \mathrm{H}_{32} \mathrm{OSi}$ : C, 79.06; H, 8.85. Found: C, 79.27; H, 8.93.

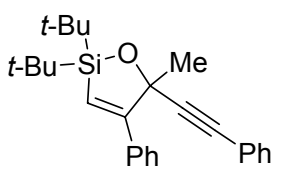

3e

\section{2,2-Di-tert-butyl-4-phenyl-5-phenylethynyl-5-methyloxasilacyclopentene (3e).}

The representative procedure for the two-step, one flask formation of oxasilacyclopentenes was followed with phenylacetylene $(0.110 \mathrm{~mL}, 1.00 \mathrm{mmol}), 1(0.225 \mathrm{~g}, 1.00 \mathrm{mmol})$ and $\mathrm{Ag}_{3} \mathrm{PO}_{4}(0.042 \mathrm{~g}, 0.100 \mathrm{mmol})$ for $3 \mathrm{hours}$ and 4-phenyl-3-butyn-2-one $(0.437 \mathrm{~mL}, 3.00 \mathrm{mmol})$ and $\mathrm{CuBr}_{2}(0.033 \mathrm{~g}, 0.148 \mathrm{mmol})$ for 1 hour to provide a $\geq 99: 1$ unpurified mixture of regioisomers. Purification by column chromatography $\left(15: 85 \mathrm{CH}_{2} \mathrm{Cl}_{2}\right.$ :hexanes) gave 3e $(0.311 \mathrm{~g}, 80 \%)$ as a pale yellow oil: $\mathrm{GC} \underline{t}_{\mathrm{R}}=15.09\left(100-250{ }^{\circ} \mathrm{C}, 10{ }^{\circ} \mathrm{C} / \mathrm{min}\right) ;{ }^{1} \mathrm{H} \mathrm{NMR}\left(\mathrm{CDCl}_{3}, 500\right.$ $\mathrm{MHz}) \delta 7.76(\mathrm{~m}, 2 \mathrm{H}), 7.44(\mathrm{~m}, 2 \mathrm{H}), 7.32(\mathrm{~m}, 6 \mathrm{H}), 6.20(\mathrm{~s}, 1 \mathrm{H}), 1.72(\mathrm{~s}, 3 \mathrm{H}), 1.16(\mathrm{~s}, 9 \mathrm{H}), 1.10(\mathrm{~s}, 9 \mathrm{H}) ;{ }^{13} \mathrm{C}$ $\mathrm{NMR}\left(\mathrm{CDCl}_{3}, 125 \mathrm{MHz}\right) \delta 164.9,138.3,131.7,128.5,128.4,128.3,128.2,128.0,123.4,122.7,92.9,85.2$, 81.3, 30.0, 28.4, 28.2, 20.9, 20.5; IR (thin film) 2963, 2857, 1598, 1472, 1092, 957, $824 \mathrm{~cm}^{-1}$; HRMS (NH $\left.{ }_{3} \mathrm{CI}\right)$ $m / z$ calcd for $\mathrm{C}_{26} \mathrm{H}_{32} \mathrm{OSi}(\mathrm{M})^{+}$388.2222, found 388.2222. Anal. Calcd for $\mathrm{C}_{26} \mathrm{H}_{32} \mathrm{OSi}$ : C, 80.36; $\mathrm{H}, 8.30$. Found: C, 80.46; H, 8.32.

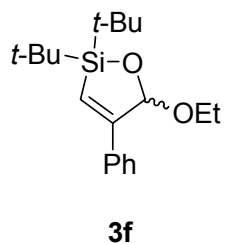

\section{2,2-Di-tert-butyl-4-phenyl-5-ethoxyoxasilacyclopentene (3f).}

The representative procedure for the two-step, one flask formation of oxasilacyclopentenes was followed with phenylacetylene $(0.110 \mathrm{~mL}, 1.00 \mathrm{mmol}), 1(0.225 \mathrm{~g}, 1.00 \mathrm{mmol})$ and $\mathrm{Ag}_{3} \mathrm{PO}_{4}(0.042 \mathrm{~g}, 0.100 \mathrm{mmol})$ for $3 \mathrm{hours}$ and ethyl formate $(0.242 \mathrm{~mL}, 3.00 \mathrm{mmol})$ and $\mathrm{CuBr}_{2}(0.033 \mathrm{~g}, 0.148 \mathrm{mmol})$ for 1 hour to provide a $\geq 99: 1$ unpurified mixture of regioisomers. Purification by column chromatography $\left(10: 90 \mathrm{CH}_{2} \mathrm{Cl}_{2}:\right.$ hexanes to $15: 95$ $\mathrm{CH}_{2} \mathrm{Cl}_{2}$ :hexanes) gave $3 \mathbf{f}$ as a colorless oil $(0.217 \mathrm{~g}, 68 \%)$ : GC $\underline{t}_{\mathrm{R}}=16.43\left(50-290{ }^{\circ} \mathrm{C}, 10{ }^{\circ} \mathrm{C} / \mathrm{min}\right) ;{ }^{1} \mathrm{H} \mathrm{NMR}$ $\left(\mathrm{CDCl}_{3}, 500 \mathrm{MHz}\right) \delta 7.59(\mathrm{~m}, 2 \mathrm{H}), 7.35(\mathrm{~m}, 2 \mathrm{H}), 7.29(\mathrm{~m}, 1 \mathrm{H}), 6.54(\mathrm{~d}, J=0.8,1 \mathrm{H}), 5.98(\mathrm{~d}, J=0.8,1 \mathrm{H}), 3.97$ $(\mathrm{dq}, J=9.7,7.1,1 \mathrm{H}), 3.75(\mathrm{dq}, J=9.6,7.1,1 \mathrm{H}), 1.26(\mathrm{t}, J=7.1,3 \mathrm{H}), 1.08(\mathrm{~s}, 9 \mathrm{H}), 1.02(\mathrm{~s}, 9 \mathrm{H}) ;{ }^{13} \mathrm{C}$ NMR $\left(\mathrm{CDCl}_{3}, 125 \mathrm{MHz}\right) \delta 158.7,135.7,128.7,128.5,126.8,123.0,104.4,63.6,28.2,27.7,20.7,20.2$, 15.7; IR (thin film) $2961,2930,2857,1472,1030,824 \mathrm{~cm}^{-1}$; HRMS $\left(\mathrm{NH}_{3} \mathrm{CI}\right) \mathrm{m} / \mathrm{z}$ calcd for $\mathrm{C}_{19} \mathrm{H}_{30} \mathrm{O}_{2} \mathrm{Si}(\mathrm{M}){ }^{+} 318.2015$, found 318.2010. Anal. Calcd for $\mathrm{C}_{19} \mathrm{H}_{30} \mathrm{O}_{2} \mathrm{Si}$ : C, 71.64; H, 9.49. Found: C, 71.41; H, 9.50. 


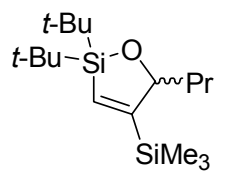

4

\section{2,2-Di-tert-butyl-4-trimethylsilyl-5-propyloxasilacyclopentene (4).}

The representative procedure for the two-step, one flask formation of oxasilacyclopentenes was followed with trimethylsilylacetylene $(0.141 \mathrm{~mL}, 1.00 \mathrm{mmol}), 1(0.225 \mathrm{~g}, 1.00 \mathrm{mmol})$ and $\mathrm{Ag}_{3} \mathrm{PO}_{4}(0.042 \mathrm{~g}, 0.100 \mathrm{mmol})$ for 3 hours and butyraldehyde $(0.270 \mathrm{~mL}, 3.00 \mathrm{mmol})$ and $\mathrm{CuI}(0.029 \mathrm{~g}, 0.152 \mathrm{mmol})$ for 10 hours to provide a 96:4 unpurified mixture of regioisomers. Purification by column chromatography $\left(7: 93 \mathrm{CH}_{2} \mathrm{Cl}_{2}\right.$ :hexanes $)$ gave 4 $(0.243 \mathrm{~g}, 78 \%)$ as a colorless oil: $\mathrm{GC} \underline{t}_{\mathrm{R}}=12.39\left(50-290{ }^{\circ} \mathrm{C}, 10{ }^{\circ} \mathrm{C} / \mathrm{min}\right) ;{ }^{1} \mathrm{H} \mathrm{NMR}\left(\mathrm{CDCl}_{3}, 500 \mathrm{MHz}\right) \delta 6.36(\mathrm{~d}$, $J=2.2,1 \mathrm{H}), 4.73(\mathrm{dt}, J=2.5,10.0,1 \mathrm{H}), 1.69(\mathrm{~m}, 1 \mathrm{H}), 1.60(\mathrm{~m}, 1 \mathrm{H}), 1.50(\mathrm{~m}, 1 \mathrm{H}), 1.29(\mathrm{~m}, 1 \mathrm{H}), 0.99(\mathrm{~s}, 9 \mathrm{H})$, 0.95-0.93 (t, $J=7.4,3 \mathrm{H} ; \mathrm{s}, 9 \mathrm{H}), 0.13(\mathrm{~s}, 9 \mathrm{H}) ;{ }^{13} \mathrm{C} \mathrm{NMR}\left(\mathrm{CDCl}_{3}, 125 \mathrm{MHz}\right) \delta 172.1,135.7,87.7,39.6,28.3$, 27.7, 21.4, 19.8, 19.8, 14.3, -0.5; IR (thin film) 2958, 2856, 1472, 1250, 1005, $823 \mathrm{~cm}^{-1} ; \mathrm{HRMS}\left(\mathrm{NH}_{3} \mathrm{CI}\right) \mathrm{m} / z$ calcd for $\mathrm{C}_{17} \mathrm{H}_{35} \mathrm{OSSi}_{2}(\mathrm{M}-\mathrm{H})^{+} 311.2227$, found 311.2235. Anal. Calcd for $\mathrm{C}_{17} \mathrm{H}_{36} \mathrm{OSi}_{2}$ : C, 65.31; $\mathrm{H}, 11.61$. Found: C, 65.03; H, 11.64.

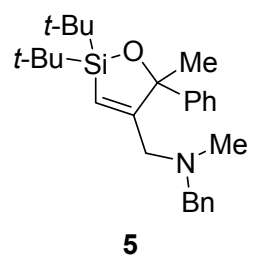

\section{$N$-Benzyl- $N$-(2,2-Di-tert-butyl-5-phenyl-5-methyl-4-methyloxasilacyclopentenyl)methylamine (5).}

To a vessel fitted with an air-free seal containing $\mathrm{Ag}_{3} \mathrm{PO}_{4}(0.042 \mathrm{~g}, 0.100 \mathrm{mmol})$ and $\mathrm{C}_{7} \mathrm{H}_{8}(4.0 \mathrm{~mL})$ was added $N$-methyl- $N$-propargylbenzylamine $(0.169 \mathrm{~mL}, 1.00 \mathrm{mmol})$ by syringe, followed by $1(0.225 \mathrm{~g}, 1.00 \mathrm{mmol})$. The reaction mixture was heated to $80{ }^{\circ} \mathrm{C}$ for $40 \mathrm{~h}$, then the reaction mixture was cooled to $22{ }^{\circ} \mathrm{C}$. Acetophenone $(0.350 \mathrm{~mL}, 3.00 \mathrm{mmol})$ was added, followed by $\mathrm{CuI}(0.058 \mathrm{~g}, 0.30 \mathrm{mmol})$. The reaction mixture was then heated to $110{ }^{\circ} \mathrm{C}$ for 3 hours. The reaction mixture was then cooled to $22{ }^{\circ} \mathrm{C}$ and filtered through a short column of Davasil ${ }^{\circledR}$ with hexanes and concentrated in vacuo to give a colorless oil as a 90:10 mixture of regioisomers. Purification by column chromatography $\left(50: 50 \mathrm{CHCl}_{3}\right.$ :hexanes) gave $5(0.305 \mathrm{~g}, 72 \%)$ as a yellow oil: $\mathrm{GC} \underline{t}_{\mathrm{R}}=22.90\left(50-290{ }^{\circ} \mathrm{C}, 10{ }^{\circ} \mathrm{C} / \mathrm{min}\right) ;{ }^{1} \mathrm{H}$ NMR $\left(\mathrm{CDCl}_{3}, 500 \mathrm{MHz}\right) \delta 7.43(\mathrm{~m}, 2 \mathrm{H}), 7.34(\mathrm{~m}, 4 \mathrm{H})$, $7.25(\mathrm{~m}, 3 \mathrm{H}), 7.20(\mathrm{~m}, 2 \mathrm{H}), 6.33(\mathrm{t}, J=1.5,1 \mathrm{H}), 3.52(\mathrm{~d}, J=3.1,2 \mathrm{H}), 3.14(\mathrm{dd}, J=4.8,1.5,1 \mathrm{H}), 2.25(\mathrm{~s}, 3 \mathrm{H})$, $1.77(\mathrm{~s}, 3 \mathrm{H}), 1.06(\mathrm{~s}, 9 \mathrm{H}), 0.89(\mathrm{~s}, 9 \mathrm{H}) ;{ }^{13} \mathrm{C} \mathrm{NMR}\left(\mathrm{CDCl}_{3}, 125 \mathrm{MHz}\right) \delta 165.8,145.6,139.6,129.0,128.5,127.9$, 127.2, 127.1, 126.8, 121.5, 88.2, 62.5, 59.8, 42.9, 28.9, 28.7, 28.6, 20.8, 20.7; IR (thin film) 2934, 2857, 1495, $822 \mathrm{~cm}^{-1}$; HRMS (ES) $\mathrm{m} / \mathrm{z}$ calcd for $\mathrm{C}_{27} \mathrm{H}_{40} \mathrm{NOSi}(\mathrm{M}+\mathrm{H})^{+} 422.2879$, found 422.2882. Anal. Calcd for $\mathrm{C}_{27} \mathrm{H}_{39}$ NOSi: C, 76.90; H, 9.32; N, 3.32. Found: C, 76.66; H, 9.49; N, 3.31.

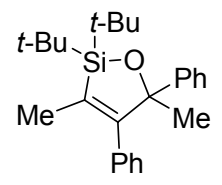

6a

\section{2,2-Di-tert-butyl-3-methyl-4-phenyl-5-phenyl-5-methyloxasilacyclopentene (6a).}

The representative procedure for the two-step, one flask formation of oxasilacyclopentenes was followed with 1-phenyl-1-propyne $(0.125 \mathrm{~mL}, 1.00 \mathrm{mmol}), 1(0.225 \mathrm{~g}, 1.00 \mathrm{mmol})$ and $\mathrm{Ag}_{3} \mathrm{PO}_{4}(0.042 \mathrm{~g}, 0.100 \mathrm{mmol})$ for 1.5 hours and acetophenone $(0.350 \mathrm{~mL}, 3.00 \mathrm{mmol})$ and $\mathrm{Cu}(\mathrm{OTf})_{2}(0.054 \mathrm{~g}, 0.149 \mathrm{mmol})$ for 1 hour to provide a 97:3 unpurified mixture of regioisomers. Purification by column chromatography $\left(12: 88 \mathrm{CH}_{2} \mathrm{Cl}_{2}:\right.$ hexanes $)$ gave 6a $(0.355 \mathrm{~g}, 94 \%)$ as a white solid. Crystallization by slow evaporation of $\mathrm{CH}_{2} \mathrm{Cl}_{2}$ and $\mathrm{CH}_{3} \mathrm{CN}$ afforded crystals suitable for X-ray crystallographic analysis (crystallographc data is provided, vide infra): mp 103-104 ${ }^{\circ} \mathrm{C}$; GC $t_{\mathrm{R}}=13.51\left(100-250{ }^{\circ} \mathrm{C}, 10{ }^{\circ} \mathrm{C} / \mathrm{min}\right) ;{ }^{1} \mathrm{H}$ NMR $\left(\mathrm{CDCl}_{3}, 500 \mathrm{MHz}\right) \delta 7.32(\mathrm{~m}, 3 \mathrm{H}), 7.17(\mathrm{~m}, 5 \mathrm{H}), 6.91$ $(\mathrm{m}, 2 \mathrm{H}), 1.78(\mathrm{~s}, 3 \mathrm{H}), 1.62(\mathrm{~s}, 3 \mathrm{H}), 1.22(\mathrm{~s}, 9 \mathrm{H}), 1.13(\mathrm{~s}, 9 \mathrm{H}) ;{ }^{13} \mathrm{C} \mathrm{NMR}\left(\mathrm{CDCl}_{3}, 125 \mathrm{MHz}\right) \delta 161.0,145.6$, $139.0,130.8,130.0,128.1,127.2,127.0,126.7,126.4,89.2,31.5,29.0,28.7,21.3,21.1,16.1$; IR (thin film) 
2932, 2857, 1473, 938, $822 \mathrm{~cm}^{-1}$; HRMS $\left(\mathrm{NH}_{3} \mathrm{CI}\right) \mathrm{m} / \mathrm{z}$ calcd for $\mathrm{C}_{25} \mathrm{H}_{35} \mathrm{OSi}(\mathrm{M}+\mathrm{H})^{+} 379.2457$, found 379.2464. Anal. Calcd for $\mathrm{C}_{25} \mathrm{H}_{34} \mathrm{OSi}$ : C, 79.31; H, 9.05. Found: C, 79.39; H, 9.17.

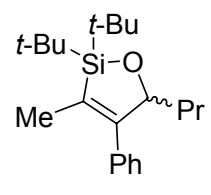

6b

2,2-Di-tert-butyl-3-methyl-4-phenyl-5-propyloxasilacyclopentene (6b).

The representative procedure for the two-step, one flask formation of oxasilacyclopentenes was followed with 1-phenyl-1-propyne $(0.168 \mathrm{~mL}, 1.34 \mathrm{mmol}), 1(0.300 \mathrm{~g}, 1.34 \mathrm{mmol})$ and $\mathrm{Ag}_{3} \mathrm{PO}_{4}(0.056 \mathrm{~g}, 0.134 \mathrm{mmol})$ for 1.5 hours and butyraldehyde $(0.362 \mathrm{~mL}, 4.02 \mathrm{mmol})$ and $\mathrm{Cu}(\mathrm{OTf})_{2}(0.073 \mathrm{~g}, 0.202 \mathrm{mmol})$ for 13 hours to provide a 95:5 unpurified mixture of regioisomers. Purification by column chromatography $\left(5: 95 \mathrm{CH}_{2} \mathrm{Cl}_{2}\right.$ :hexanes $)$ gave 6b $(0.237 \mathrm{~g}, 54 \%)$ as a colorless oil: $\mathrm{GC} t_{\mathrm{R}}=16.57$ (major), 16.82 (minor) $\left(50-290{ }^{\circ} \mathrm{C}, 10{ }^{\circ} \mathrm{C} / \mathrm{min}\right) ;{ }^{1} \mathrm{H} \mathrm{NMR}$ $\left(\mathrm{CDCl}_{3}, 500 \mathrm{MHz}\right) \delta 7.34(\mathrm{~m}, 2 \mathrm{H}), 7.26(\mathrm{~m}, 1 \mathrm{H}), 7.07(\mathrm{~m}, 2 \mathrm{H}), 4.82(\mathrm{ddd}, J=9.3,3.1,1.9,1 \mathrm{H}), 1.77(\mathrm{~s}, 3 \mathrm{H})$, $1.50(\mathrm{~m}, 1 \mathrm{H}), 1.37(\mathrm{~m}, 2 \mathrm{H}), 1.24(\mathrm{~m}, 1 \mathrm{H}), 1.10(\mathrm{~s}, 9 \mathrm{H}), 1.06(\mathrm{~s}, 9 \mathrm{H}), 0.82(\mathrm{t}, J=7.3,3 \mathrm{H}) ;{ }^{13} \mathrm{C} \mathrm{NMR}\left(\mathrm{CDCl}_{3}\right.$, $125 \mathrm{MHz}) \delta 158.1,138.5,129.2,128.4,128.4,127.0,85.3,38.7,28.3,27.9,22.1,20.5,19.6,15.6,14.3$; IR (thin film) 2931, 2856, 1591, 1474, $822 \mathrm{~cm}^{-1}$; HRMS $\left(\mathrm{NH}_{3} \mathrm{CI}\right) \mathrm{m} / \mathrm{z}$ calcd for $\mathrm{C}_{21} \mathrm{H}_{34} \mathrm{OSi}(\mathrm{M})^{+} 330.2379$, found 330.2376. Anal. Calcd for $\mathrm{C}_{21} \mathrm{H}_{34} \mathrm{OSi}$ : C, 76.30; H, 10.37. Found: C, 76.18; H, 10.27.

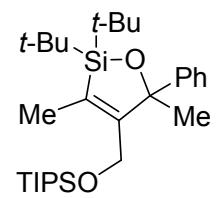

7

2,2-Di-tert-butyl-3-methyl-4-triisopropylsiloxymethyl-5-methyl-5-phenyloxasilacyclopentene (7).

The representative procedure for the two-step, one flask formation of oxasilacyclopentenes was followed with alkyne 4-triisopropylsilyloxy-2-butyne $(0.227 \mathrm{~g}, 1.00 \mathrm{mmol}), 1(0.225 \mathrm{~g}, 1.00 \mathrm{mmol})$ and $\mathrm{Ag}_{3} \mathrm{PO}_{4}(0.042 \mathrm{~g}$, $0.100 \mathrm{mmol})$ for 3 hours and acetophenone $(0.350 \mathrm{~mL}, 3.00 \mathrm{mmol})$ and $\mathrm{Cu}(\mathrm{OTf})_{2}(0.054 \mathrm{~g}, 0.149 \mathrm{mmol})$ for 13 hours to provide a 99:1 unpurified mixture of regioisomers. Purification by column chromatography $(15: 85$ $\mathrm{CH}_{2} \mathrm{Cl}_{2}$ :hexanes) gave $7(0.447 \mathrm{~g}, 91 \%)$ as a colorless oil: $\mathrm{GC} t_{\mathrm{R}}=23.17\left(50-290{ }^{\circ} \mathrm{C}, 10{ }^{\circ} \mathrm{C} / \mathrm{min}\right) ;{ }^{1} \mathrm{H} \mathrm{NMR}$ $\left(\mathrm{CDCl}_{3}, 500 \mathrm{MHz}\right) \delta 7.43(\mathrm{~m}, 2 \mathrm{H}), 7.25(\mathrm{~m}, 2 \mathrm{H}), 7.19(\mathrm{~m}, 1 \mathrm{H}), 4.57(\mathrm{~d}, J=11.5,1 \mathrm{H}), 4.27(\mathrm{~d}, J=11.6,1 \mathrm{H})$, $2.03(\mathrm{~s}, 3 \mathrm{H}), 1.78(\mathrm{~s}, 3 \mathrm{H}), 1.15-1.04(\mathrm{~m}, 30 \mathrm{H}), 0.81(\mathrm{~s}, 9 \mathrm{H}) ;{ }^{13} \mathrm{C} \mathrm{NMR}\left(\mathrm{CDCl}_{3}, 125 \mathrm{MHz}\right) \delta 157.2,147.1$, $133.9,128.0,127.2,127.1,89.1,59.6,29.5,28.9,28.8,21.2,21.1,18.7,18.6,12.5$; IR (thin film) 2942, 2865, 1602, 1463, 1099, $822 \mathrm{~cm}^{-1}$; HRMS $\left(\mathrm{NH}_{3} \mathrm{CI}\right) \mathrm{m} / \mathrm{z}$ calcd for $\mathrm{C}_{29} \mathrm{H}_{51} \mathrm{O}_{2} \mathrm{Si}_{2}(\mathrm{M}-\mathrm{H})^{+} 487.3422$, found 487.3418 . Anal. Calcd for $\mathrm{C}_{29} \mathrm{H}_{52} \mathrm{O}_{2} \mathrm{Si}_{2}: \mathrm{C}, 71.24 ; \mathrm{H}, 10.72$. Found: $\mathrm{C}, 71.34 ; \mathrm{H}, 10.87$.

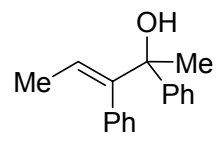

2,3-Diphenyl-3-penten-2-ol (8).

8

To a mixture of KOt-Bu $(0.133 \mathrm{~g}, 1.19 \mathrm{mmol})$, 1-methyl-2-pyrrolidinone $(3.0 \mathrm{~mL})$, THF $(2.0 \mathrm{~mL})$ and toluene $(2.0 \mathrm{~mL})$ was added $8(0.150 \mathrm{~g}, 0.396 \mathrm{mmol})$, followed by $1.19 \mathrm{~mL}$ of a $1.0 \mathrm{M}$ solution of TBAF in THF. After 13 hours, a second portion of TBAF $(1.19 \mathrm{~mL})$ was added. After 31 hours, the reaction mixture was diluted with $\mathrm{H}_{2} \mathrm{O}(15 \mathrm{~mL})$ and extracted with MTBE $(3 \times 15 \mathrm{~mL})$. The organic extracts were rinsed with $\mathrm{H}_{2} \mathrm{O}(3 \times 10 \mathrm{~mL})$, dried with $\mathrm{Na}_{2} \mathrm{SO}_{4}$, and concentrated in vacuo. Purification by column chromatography $\left(80: 20 \mathrm{CH}_{2} \mathrm{Cl}_{2}\right.$ :hexanes) provided $8(0.079 \mathrm{~g}, 84 \%)$ as a colorless oil: ${ }^{1} \mathrm{H}$ NMR $\left(\mathrm{CDCl}_{3}, 500 \mathrm{MHz}\right) \delta 7.48(\mathrm{~m}, 2 \mathrm{H}), 7.36(\mathrm{~m}, 2 \mathrm{H}), 7.30$ $(\mathrm{m}, 1 \mathrm{H}), 7.25(\mathrm{~m}, 3 \mathrm{H}), 6.83(\mathrm{~m}, 2 \mathrm{H}), 6.02(\mathrm{q}, J=6.8,1 \mathrm{H}), 1.99($ br s, $1 \mathrm{H}), 1.73(\mathrm{~s}, 3 \mathrm{H}), 1.50(\mathrm{~d}, J=6.8,3 \mathrm{H})$; ${ }^{3} \mathrm{C}$ NMR $\left(\mathrm{CDCl}_{3}, 125 \mathrm{MHz}\right) \delta 147.7,147.0,138.2,130.2,128.1,128.1,127.1,126.9,126.0,122.2,77.4,29.6$, 15.0; IR (thin film) 3459, 3055, 2979, 2859, 1600, $1492 \mathrm{~cm}^{-1}$; LRMS (EI/GCMS) $m / z$ calcd for $\mathrm{C}_{17} \mathrm{H}_{16}(\mathrm{M}-$ $\left.\mathrm{H}_{2} \mathrm{O}\right)^{+} 220$, found 220. Anal. Calcd for $\mathrm{C}_{17} \mathrm{H}_{18} \mathrm{O}: \mathrm{C}, 85.67$; H, 7.61. Found: C, 85.41; H, 7.74. 
Diol 9 was constructed in two-steps from oxasilacyclopenene $\mathbf{6 b}$ (Scheme S-1). Hydrogenation of $\mathbf{6 b}$ provided intermediate A in 99:1 diastereoselectivity. Oxidation of A provided the 1,3-diol 9. Proof of stereochemistry was established by formation and analysis of acetal B. Experimental procedures for these transformations follow:

\section{Scheme S-1}

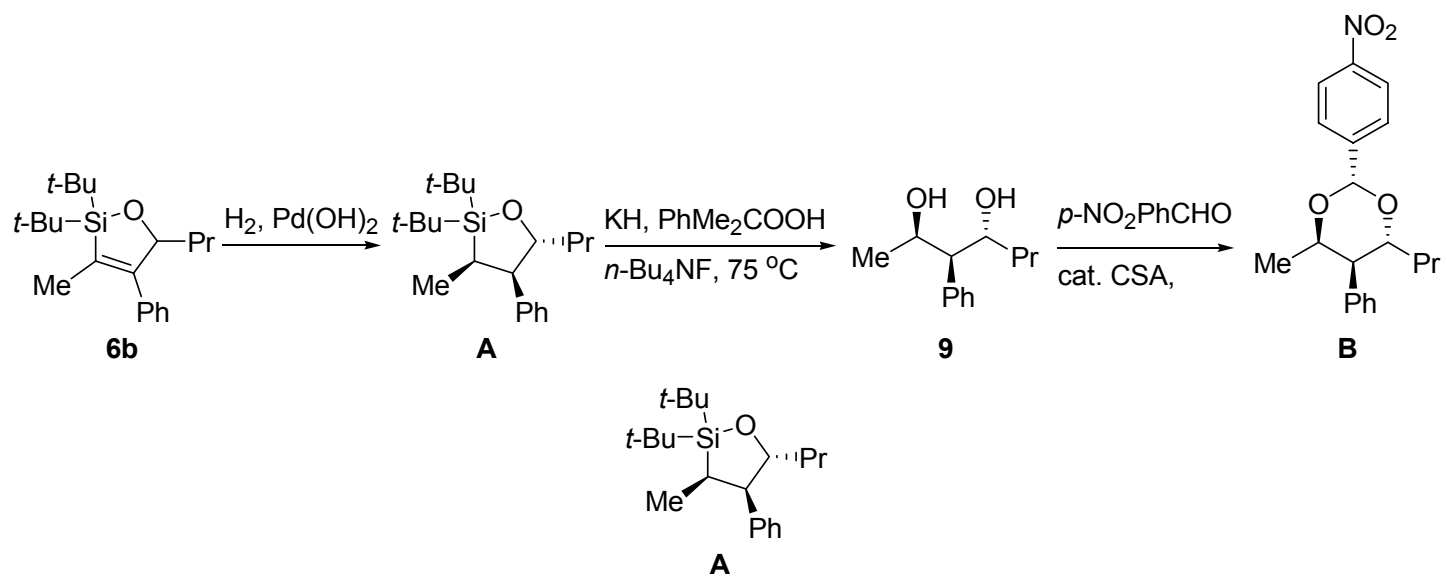

$\left(3 R^{*}, 4 R^{*}, 5 R^{*}\right)$-2,2-Di-tert-butyl-3-methyl-4-phenyl-5-propyloxasilacyclopentane (A).

To an oven-dried $50 \mathrm{~mL}$ round-bottom flask equipped with a magnetic stir-bar was added $\mathbf{6 b}(0.134 \mathrm{~g}, 0.405$ $\mathrm{mmol})$ and ethanol $(20 \mathrm{~mL})$. The reaction mixture was sparged for 5 minutes with $\mathrm{H}_{2}$ gas, followed by addition of $20 \% \mathrm{Pd}(\mathrm{OH})_{2}$ on carbon $(0.057 \mathrm{~g}, 0.081 \mathrm{mmol})$. The reaction mixture was allowed to stir for 4 days under a balloon of hydrogen gas, upon which more $\mathrm{Pd}(\mathrm{OH})_{2}$ on carbon $(0.014 \mathrm{~g}, 0.020 \mathrm{mmol})$ was added. The reaction mixture was allowed to stir for 1 more day under a balloon of hydrogen gas. The reaction mixture was passed over a short column of silica and concentrated in vacuo to provide the unpurified product as a 99:1 mixture of diastereomers (as determined by gas chromatography). Purification by column chromatography (5:95 $\mathrm{CH}_{2} \mathrm{Cl}_{2}$ :hexanes $)$ provided $\mathbf{A}(0.113 \mathrm{~g}, 84 \%)$ as a clear oil: $\mathrm{GC} t_{\mathrm{R}}=17.48\left(50-290{ }^{\circ} \mathrm{C}, 10{ }^{\circ} \mathrm{C} / \mathrm{min}\right) ;{ }^{1} \mathrm{H} \mathrm{NMR}$ $\left(\mathrm{CDCl}_{3}, 500 \mathrm{MHz}\right) \delta 7.28(\mathrm{t}, J=7.5,2 \mathrm{H}), 7.19(\mathrm{t}, J=7.4,1 \mathrm{H}), 7.06(\mathrm{~d}, J=7.0,2 \mathrm{H}), 4.24(\mathrm{ddd}, J=10.5,7.7$, 2.0, $1 \mathrm{H}), 3.08(\mathrm{dd}, J=11.0,8.3,1 \mathrm{H}), 1.61(\mathrm{p}, J=8.2,1 \mathrm{H}), 1.51(\mathrm{~m}, 2 \mathrm{H}), 1.41(\mathrm{~m}, 1 \mathrm{H}), 1.30(\mathrm{~m}, 1 \mathrm{H}), 1.14(\mathrm{~s}$, $9 \mathrm{H}), 1.08(\mathrm{~s}, 9 \mathrm{H}), 0.85(\mathrm{t}, J=7.1,3 \mathrm{H}), 0.78(\mathrm{~d}, J=8.2,3 \mathrm{H}) ;{ }^{13} \mathrm{C} \mathrm{NMR}\left(\mathrm{CDCl}_{3}, 125 \mathrm{MHz}\right) \delta 140.1,129.4$, 128.4, 126.1, 78.4, 54.7, 37.8, 28.9, 28.4, 22.5, 20.9, 19.1, 14.4, 13.2; IR (thin film) 2959, 2857, 1471, 822 $\mathrm{cm}^{-1}$; HRMS (EI) $m / z$ calcd for $\mathrm{C}_{21} \mathrm{H}_{35} \mathrm{OSi}(\mathrm{M}-\mathrm{H})^{+}$331.2457, found 317.2451. Anal. Calcd for $\mathrm{C}_{21} \mathrm{H}_{36} \mathrm{OSi}$ : $\mathrm{C}$, 75.84; H, 10.91. Found: C, 76.08; H, 11.03.

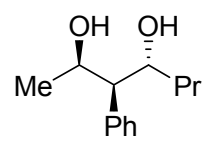

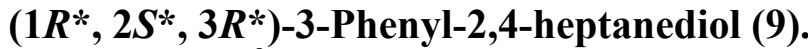

9

To a cooled $\left(0{ }^{\circ} \mathrm{C}\right)$ slurry of $\mathrm{KH}(0.031 \mathrm{~g}, 1.60 \mathrm{mmol})$ in $0.8 \mathrm{~mL}$ of 1-methyl-2-pyrrolidinone (NMP) was added tert-butylhydroperoxide $\left(0.107 \mathrm{~mL}, 0.8 \mathrm{mmol}, 70 \%\right.$ in $\left.\mathrm{H}_{2} \mathrm{O}\right)$ dropwise by syringe (no needle). A solution of $\mathbf{A}$ $(0.086 \mathrm{~g}, 0.259)$ was then added in $1.0 \mathrm{~mL}$ of a 50:50 mixture of THF:toluene followed by $2.60 \mathrm{~mL}$ of a $1.0 \mathrm{M}$ solution of TBAF in THF. The reaction was warmed $\left(75^{\circ} \mathrm{C}\right)$ for $7 \mathrm{~h}$, cooled, and diluted with $4 \mathrm{~mL}$ of a $1.3 \mathrm{M}$ aqueous solution of $\mathrm{Na}_{2} \mathrm{~S}_{2} \mathrm{O}_{3}$. The resulting mixture was extracted with MTBE $(3 \times 15 \mathrm{~mL})$. The organic extracts were washed with $\mathrm{H}_{2} \mathrm{O}(3 \times 10 \mathrm{~mL})$, dried with $\mathrm{Na}_{2} \mathrm{SO}_{4}$, and concentrated in vacuo. Purification by column chromatography (1:99 $\mathrm{MeOH}: \mathrm{CH}_{2} \mathrm{Cl}_{2}$ to 2:98 $\left.\mathrm{MeOH}: \mathrm{CH}_{2} \mathrm{Cl}_{2}\right)$ provided $9(0.048 \mathrm{~g}, 89 \%)$ as a colorless solid: $\mathrm{mp}$ 68.5-69.5 ${ }^{\circ} \mathrm{C} ;{ }^{1} \mathrm{H}$ NMR $\left(\mathrm{CDCl}_{3}, 500 \mathrm{MHz}\right) \delta 7.31(\mathrm{~m}, 2 \mathrm{H}), 7.25(\mathrm{~m}, 3 \mathrm{H}), 4.42(\mathrm{dq}, J=6.5,3.0,1 \mathrm{H})$, $4.17(\mathrm{~m}, 1 \mathrm{H}), 2.70$ (br s, $1 \mathrm{H}), 2.65-2.59$ (br s, $1 \mathrm{H}$; dd, $J=8.7,3.1,1 \mathrm{H}), 1.50(\mathrm{~m}, 1 \mathrm{H}), 1.32(\mathrm{~m}, 3 \mathrm{H}), 1.06(\mathrm{~d}, J$ 
$=6.5,3 \mathrm{H}), 0.84(\mathrm{t}, J=7.1,3 \mathrm{H}) ;{ }^{13} \mathrm{C} \mathrm{NMR}\left(\mathrm{CDCl}_{3}, 125 \mathrm{MHz}\right) \delta 139.5,129.8,128.6,127.0,73.1,68.3,57.6$, 38.1, 21.0, 19.0, 14.1; IR (thin film) 3382 (br), 2960, 1454, $1138 \mathrm{~cm}^{-1}$; HRMS: (EI/GCMS) $\mathrm{m} / \mathrm{z}$ calcd for $\mathrm{C}_{13} \mathrm{H}_{18} \mathrm{O}\left(\mathrm{M}-\mathrm{H}_{2} \mathrm{O}\right)^{+}$190.1358, found 190.1352. Anal. Calcd for $\mathrm{C}_{13} \mathrm{H}_{20} \mathrm{O}_{2}: \mathrm{C}, 74.96 ; \mathrm{H}, 9.68$. Found: C, 74.52; $\mathrm{H}, 9.64$.

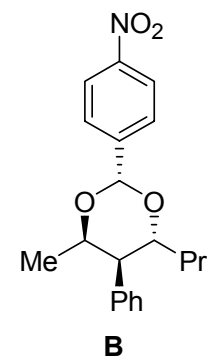

$\left(2 R^{*}, 4 R^{*}, 5 S^{*}, 6 R^{*}\right)-4-M e t h y l-2-($ nitrophenyl)-5-phenyl-6-propyl-1,3-dioxane (B).

A flask fitted with a Dean-Stark trap and reflux condenser was charged with diol 9 (0.029 g, 0.139 mmol) and 4-nitrobenzaldehyde $(0.021 \mathrm{~g}, 0.139 \mathrm{mmol})$ in $9 \mathrm{~mL}$ of benzene. CSA $(<0.005 \mathrm{~g})$ was added and the solution was heated to reflux for $22 \mathrm{~h}$. The solution was cooled to $22{ }^{\circ} \mathrm{C}$ and $2-3$ drops of $\mathrm{Et}_{3} \mathrm{~N}$ were added. The solution was concentrated in vacuo to afford a yellow oil as a 63:37 mixture of diastereomers. Purification by column chromatography (18:82 $\mathrm{CH}_{2} \mathrm{Cl}_{2}$ :hexanes to $20: 80 \mathrm{CH}_{2} \mathrm{Cl}_{2}$ :hexanes) gave $\mathbf{B}(0.046 \mathrm{~g}, 97 \%)$ as a colorless oil in three portions: the major isomer as a 99:1 mixture of diastereomers $(0.0196 \mathrm{~g})$; the minor isomer as a 94:6 mixture of diastereomers $(0.0102 \mathrm{~g})$; and a 55:45 mixture of diastereomers $(0.016 \mathrm{~g})$ : mixture: IR (thin film) 2933, 2871, 1608, 1523, 1348, $1146 \mathrm{~cm}^{-1}$; HRMS (EI/GCMS) $m / z$ calcd for $\mathrm{C}_{20} \mathrm{H}_{22} \mathrm{NO}_{4}(\mathrm{M}-\mathrm{H})^{+}$ 340.1543, found 340.1546. Anal. Calcd for $\mathrm{C}_{20} \mathrm{H}_{23} \mathrm{NO}_{4}$ : C, 70.36; H, 6.79; N, 4.10. Found: C, 70.16; H, 6.95; $\mathrm{N}$, 4.05. major isomer: $\mathrm{GC} t_{\mathrm{R}}=15.51\left(100-250{ }^{\circ} \mathrm{C}, 10{ }^{\circ} \mathrm{C} / \mathrm{min}\right) ;{ }^{1} \mathrm{H} \mathrm{NMR}\left(\mathrm{CDCl}_{3}, 500 \mathrm{MHz}\right) \delta 8.27(\mathrm{dt}, J=$ 8.9, 2.1, 2H), $7.77(\mathrm{~m}, 2 \mathrm{H}), 7.50(\mathrm{~d}, J=7.0,2 \mathrm{H}), 7.30(\mathrm{~m}, 2 \mathrm{H}), 7.26(\mathrm{~m}, 1 \mathrm{H}), 5.99(\mathrm{~s}, 1 \mathrm{H}), 4.55(\mathrm{dtd}, J=6.4$, $6.4,3.3,1 \mathrm{H}), 4.25(\mathrm{dd}, J=9.6,5.7,1 \mathrm{H}), 2.47(\mathrm{~d}, J=2.7,1 \mathrm{H}), 2.26(\mathrm{dtd}, J=14.8,9.5,5.2,1 \mathrm{H}), 1.70(\mathrm{~m}, 1 \mathrm{H})$, $1.57(\mathrm{~m}, 1 \mathrm{H}), 1.46(\mathrm{~m}, 1 \mathrm{H}), 1.03(\mathrm{~d}, J=6.0,3 \mathrm{H}), 0.99(\mathrm{t}, J=7.4,3 \mathrm{H}) ;{ }^{13} \mathrm{C} \mathrm{NMR}\left(\mathrm{CDCl}_{3}, 125 \mathrm{MHz}\right)$ $\delta 148.3,146.0,141.2,129.6,128.4,127.6,127.0,123.8,93.9,78.6,71.5,48.0,33.4,19.7,19.5$, 14.1. minor isomer: $\mathrm{GC} \underline{t}_{\mathrm{R}}=15.87\left(100-250{ }^{\circ} \mathrm{C}, 10{ }^{\circ} \mathrm{C} / \mathrm{min}\right) ;{ }^{1} \mathrm{H} \mathrm{NMR}\left(\mathrm{CDCl}_{3}, 500 \mathrm{MHz}\right) \delta 8.24(\mathrm{dt}, J=2.1,8.9,2 \mathrm{H}), 7.73$ $(\mathrm{m}, 2 \mathrm{H}), 7.33(\mathrm{t}, J=7.4,2 \mathrm{H}), 7.26(\mathrm{~m}, 1 \mathrm{H}), 7.13(\mathrm{~m}, 2 \mathrm{H}), 6.02(\mathrm{~s}, 1 \mathrm{H}), 4.46(\mathrm{~m}, 2 \mathrm{H}), 3.28(\mathrm{dd}, J=5.7,10.9$, $1 \mathrm{H}), 1.51(\mathrm{~m}, 4 \mathrm{H}), 1.29(\mathrm{~d}, J=7.0,3 \mathrm{H}), 0.88(\mathrm{t}, J=7.1,3 \mathrm{H}) ;{ }^{13} \mathrm{C} \mathrm{NMR}\left(\mathrm{CDCl}_{3}, 125 \mathrm{MHz}\right) \delta 148.3,146.1$, 138.8, 129.0, 128.9, 127.5, 127.2, 123.6, 92.6, 74.9, 73.8, 50.2, 35.7, 18.6, 14.2, 13.7.

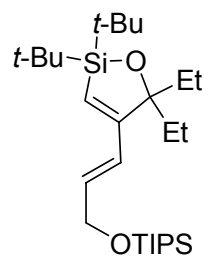

11

2,2-Di-tert-butyl-4-(3-triisopropylsilyloxy-1-propenyl)-5,5-diethyloxasilacyclopentene (11).

The representative procedure for the two-step, one flask formation of oxasilacyclopentenes was followed with alkyne 10 (0.483 g, $2.02 \mathrm{mmol}), 1(0.54 \mathrm{~g}, 2.43 \mathrm{mmol})$ and $\mathrm{Ag}_{3} \mathrm{PO}_{4}(0.085 \mathrm{~g}, 0.203 \mathrm{mmol})$ for 3 hours and 3 pentanone $(0.642 \mathrm{~mL}, 6.06 \mathrm{mmol})$ and CuI $(0.058 \mathrm{~g}, 0.305 \mathrm{mmol})$ for 12 hours to provide a $98: 2$ unpurified mixture of regioisomers. Purification by column chromatography $\left(8: 92 \mathrm{CH}_{2} \mathrm{Cl}_{2}\right.$ :hexanes to 10:90 $\mathrm{CH}_{2} \mathrm{Cl}_{2}$ :hexanes) gave $11(0.513 \mathrm{~g}, 54 \%)$ as a colorless oil: $\mathrm{GC} t_{\mathrm{R}}=21.54$ (minor), 21.71 (major), $\left(50-290{ }^{\circ} \mathrm{C}\right.$, $\left.10{ }^{\circ} \mathrm{C} / \mathrm{min}\right) ;{ }^{1} \mathrm{H} \mathrm{NMR}\left(\mathrm{CDCl}_{3}, 500 \mathrm{MHz}\right) \delta 6.23(\mathrm{~d}, J=15.5,1 \mathrm{H}), 6.11(\mathrm{dt}, J=15.4,4.3,1 \mathrm{H}), 5.87(\mathrm{~s}, 1 \mathrm{H}), 4.33$ $(\mathrm{dd}, J=4.3,1.9,2 \mathrm{H}), 1.76(\mathrm{dq}, J=14.7,7.4,2 \mathrm{H}), 1.60(\mathrm{dq}, J=15.3,7.7,2 \mathrm{H}), 1.10(\mathrm{~m}, 21 \mathrm{H}), 1.01(\mathrm{~s}, 18 \mathrm{H})$, $0.97(\mathrm{t}, J=7.5,6 \mathrm{H}) ;{ }^{13} \mathrm{C} \mathrm{NMR}\left(\mathrm{CDCl}_{3}, 125 \mathrm{MHz}\right) \delta 167.3,131.9,125.6,116.7,89.1,63.9,31.6,28.7,20.7$, 
18.2, 12.3, 9.3; IR (thin film) 2943, 2864, 1550, 1472, $822 \mathrm{~cm}^{-1}$; LRMS (EI/GCMS) $m / z$ calcd for $\mathrm{C}_{25} \mathrm{H}_{49} \mathrm{O}_{2} \mathrm{Si}_{2}\left(\mathrm{M}-\mathrm{C}_{2} \mathrm{H}_{5}\right)^{+} 437$, found 437.

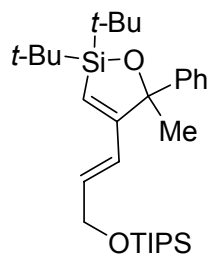

12

2,2-Di-tert-butyl-4-(3-triisopropylsiloxy-1-propenyl)-5-phenyl-5-methyloxasilacyclopentene (12).

The representative procedure for the two-step, one flask formation of oxasilacyclopentenes was followed with alkyne 10 (0.449 g, $1.88 \mathrm{mmol}), 1(0.507 \mathrm{~g}, 2.26 \mathrm{mmol})$, and $\mathrm{Ag}_{3} \mathrm{PO}_{4}(0.079 \mathrm{~g}, 0.188 \mathrm{mmol})$ for 3 hours and acetophenone $(0.658 \mathrm{~mL}, 5.64 \mathrm{mmol})$ and $\mathrm{CuI}(0.054 \mathrm{~g}, 0.282 \mathrm{mmol})$ for 2 hours to provide a 89:11 unpurified mixture of regioisomers. Purification by column chromatography $\left(10: 90 \quad \mathrm{CH}_{2} \mathrm{Cl}_{2}\right.$ :hexanes to $13: 87$ $\mathrm{CH}_{2} \mathrm{Cl}_{2}$ :hexanes) gave 12 in two portions $(0.468 \mathrm{~g}, 50 \%, 96: 4$ mixture of regioisomers, and $0.073 \mathrm{~g}, 8 \%, 23: 77$ mixture of regioisomers favoring the minor isomer) each as a colorless oil: $\mathrm{GC} \underline{t}_{\mathrm{R}}=24.42$ (major), 24.52 (minor) $\left(50-290{ }^{\circ} \mathrm{C}, 10{ }^{\circ} \mathrm{C} / \mathrm{min}\right)$. major isomer: ${ }^{1} \mathrm{H} \mathrm{NMR}\left(\mathrm{CDCl}_{3}, 500 \mathrm{MHz}\right) \delta 7.47(\mathrm{~m}, 2 \mathrm{H}), 7.24(\mathrm{~m}, 2 \mathrm{H})$, $7.18(\mathrm{~m}, 1 \mathrm{H}), 6.39(\mathrm{dt}, J=15.7,2.0,1 \mathrm{H}), 6.21(\mathrm{~s}, 1 \mathrm{H}), 6.06(\mathrm{dt}, J=15.7,4.5,1 \mathrm{H}), 4.34(\mathrm{dd}, J=4.5,1.9,2 \mathrm{H})$, $1.76(\mathrm{~s}, 3 \mathrm{H}), 1.06(\mathrm{~m}, 30 \mathrm{H}), 0.78(\mathrm{~s}, 9 \mathrm{H}) ;{ }^{13} \mathrm{C} \mathrm{NMR}\left(\mathrm{CDCl}_{3}, 125 \mathrm{MHz}\right) \delta 163.8,146.2,132.9,127.8,127.2$, 127.0, 126.3, 121.2, 88.1, 63.9, 29.5, 28.5, 28.3, 20.70, 20.69, 18.2, 12.3; IR (thin film) 2932, 2859, 1694, 1470, 943, $822 \mathrm{~cm}^{-1}$; HRMS $\left(\mathrm{NH}_{3} \mathrm{CI}\right) \mathrm{m} / \mathrm{z}$ calcd for $\mathrm{C}_{29} \mathrm{H}_{49} \mathrm{O}_{2} \mathrm{Si}_{2}\left(\mathrm{M}-\mathrm{CH}_{3}\right)^{+}$485.3271, found 485.3269. Anal. Calcd for $\mathrm{C}_{30} \mathrm{H}_{52} \mathrm{O}_{2} \mathrm{Si}_{2}$ : C, 71.93; H, 10.46. Found: C, 71.95; H, 10.63.

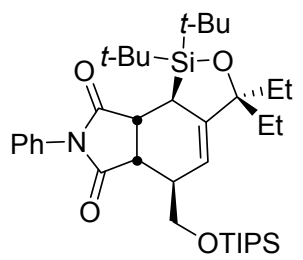

\section{Oxasilacyclopentane 13.}

13

To a vessel fitted with an air-free seal was added $11(0.459 \mathrm{~g}, 0.983 \mathrm{mmol})$ in toluene $(5.0 \mathrm{~mL})$ followed by $N$ phenylmaleimide $(0.221 \mathrm{~g}, 1.28 \mathrm{mmol})$. The vessel was sealed and heated to $130^{\circ} \mathrm{C}$ for 6 days. The reaction mixture was concentrated in vacuo to give a yellow oil as a 99:1 mixture of diastereomers (as determined by gas chromatography). Purification by column chromatography $\left(20: 80 \mathrm{CHCl}_{3}\right.$ :hexanes to $50: 50 \mathrm{CHCl}_{3}:$ hexanes) gave $13(0.475 \mathrm{~g}, 76 \%)$ as a pale yellow solid: $\mathrm{mp} 47-51{ }^{\circ} \mathrm{C}$; $\mathrm{GC} \underline{t}_{\mathrm{R}}=36.69\left(50-290{ }^{\circ} \mathrm{C}, 10{ }^{\circ} \mathrm{C} / \mathrm{min}, 35 \mathrm{~min}\right.$ at $\left.290{ }^{\circ} \mathrm{C}\right) ;{ }^{1} \mathrm{H}$ NMR $\left(\mathrm{CDCl}_{3}, 500 \mathrm{MHz}\right) \delta 7.46(\mathrm{~m}, 2 \mathrm{H}), 7.37(\mathrm{~m}, 1 \mathrm{H}), 7.31(\mathrm{~m}, 2 \mathrm{H}), 5.54(\mathrm{dd}, J=3.2,2.3,1 \mathrm{H})$, 4.06 (dd, $J=9.6,4.9,1 \mathrm{H}), 4.01(\mathrm{dd}, J=9.6,3.7,1 \mathrm{H}), 3.49(\mathrm{dd}, J=8.6,6.2,1 \mathrm{H}), 3.10(\mathrm{t}, J=8.7,1 \mathrm{H}), 2.45(\mathrm{dt}$, $J=6.4,3.7,1 \mathrm{H}), 2.37(\mathrm{~m}, 1 \mathrm{H}), 1.90(\mathrm{~h}, J=7.2,1 \mathrm{H}), 1.68(\mathrm{dq}, J=14.7,7.3,1 \mathrm{H}), 1.52(\mathrm{~m}, 1 \mathrm{H}), 1.38(\mathrm{dq}, J=$ $13.9,7.5,1 \mathrm{H}), 1.16(\mathrm{~s}, 9 \mathrm{H}), 1.08(\mathrm{~m}, 30 \mathrm{H}), 0.98(\mathrm{t}, J=7.4,3 \mathrm{H}), 0.74(\mathrm{t}, J=7.5,3 \mathrm{H}) ;{ }^{13} \mathrm{C} \mathrm{NMR}\left(\mathrm{CDCl}_{3}, 125\right.$ MHz) $\delta 179.5,178.4,146.5,132.2,129.4,128.7,126.6,118.4,84.9,64.9,40.5,39.5,38.2,31.7,29.3,28.9$, 28.6, 22.0, 21.4, 20.7, 18.3, 18.2, 12.2, 9.4, 8.2; IR (thin film) 2942, 2865, 1716, $1376 \mathrm{~cm}^{-1}$; HRMS (ES) $\mathrm{m} / \mathrm{z}$ calcd for $\mathrm{C}_{37} \mathrm{H}_{62} \mathrm{NO}_{4} \mathrm{Si}_{2}(\mathrm{M}+\mathrm{H})^{+}$640.4218, found 640.4225. Anal. Calcd for $\mathrm{C}_{37} \mathrm{H}_{61} \mathrm{NO}_{4} \mathrm{Si}_{2}: \mathrm{C}, 69.43 ; \mathrm{H}, 9.61$; N, 2.19. Found: C, 69.26; H, 9.58; N, 2.17. 


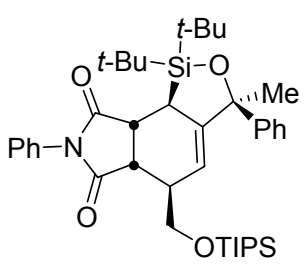

Oxasilacyclopentane 14.

14

To a vessel fitted with an air-free seal was added $12(0.230 \mathrm{~g}, 0.459 \mathrm{mmol})$ in toluene $(2.3 \mathrm{~mL})$ followed by $N$ phenylmaleimide $(0.103 \mathrm{~g}, 0.595 \mathrm{mmol})$. The vessel was sealed and heated to $130^{\circ} \mathrm{C}$ for 9 days. The reaction mixture was concentrated in vacuo to give a yellow oil as a 4:1 mixture of exo:endo diastereomers (as determined by ${ }^{1} \mathrm{H}$ NMR spectroscopy). Purification by column chromatography $\left(20: 80 \mathrm{CHCl}_{3}\right.$ :hexanes to $\left.\mathrm{CHCl}_{3}\right)$ gave 14 in two portions $(0.080 \mathrm{~g}, 26 \%$, as a 1.1:1 major:minor mixture of diastereomers as a colorless oil, and $0.132 \mathrm{~g}, 43 \%$, as a $\geq 97: 3$ mixture of diastereomers as a colorless solid). Crystallization by slow evaporation of $\mathrm{CHCl}_{3}$ and $\mathrm{CH}_{3} \mathrm{CN}$ afforded crystals suitable for X-ray crystallographic analysis (vide infra): major isomer: ${ }^{1} \mathrm{H} N M R\left(\mathrm{CDCl}_{3}, 500 \mathrm{MHz}\right) \delta 7.59(\mathrm{~d}, J=7.2,2 \mathrm{H}), 7.45(\mathrm{~m}, 3 \mathrm{H}), 7.31(\mathrm{~m}, 3 \mathrm{H}), 7.25(\mathrm{~m}, 2 \mathrm{H})$, $5.70(\mathrm{t}, J=2.5,1 \mathrm{H}), 4.07(\mathrm{dd}, J=9.7,4.5,1 \mathrm{H}), 3.95(\mathrm{dd}, J=9.6,3.3,1 \mathrm{H}), 3.52(\mathrm{dd}, J=8.8,7.0,1 \mathrm{H}), 3.13(\mathrm{t}, J$ $=9.1,1 \mathrm{H}), 2.57(\mathrm{p}, J=3.4,1 \mathrm{H}), 2.34(\mathrm{~m}, 1 \mathrm{H}), 1.70(\mathrm{~s}, 3 \mathrm{H}), 1.22(\mathrm{~s}, 9 \mathrm{H}), 1.05(\mathrm{~s}, 9 \mathrm{H}), 0.88(\mathrm{~m}, 21 \mathrm{H}) ;{ }^{13} \mathrm{C} \mathrm{NMR}$ $\left(\mathrm{CDCl}_{3}, 125 \mathrm{MHz}\right) \delta 179.1,178.3,146.8,145.8,132.1,129.4,128.7,128.0,126.9,126.6,126.3,121.6,84.4$, 64.2, 40.6, 40.3, 39.1, 32.7, 28.8, 28.6, 28.3, 22.4, 20.9, 18.2, 18.1, 12.1; IR (thin film) 2940, 2863, 1716,1376 $\mathrm{cm}^{-1}$; HRMS (ES) $m / z$ calcd for $\mathrm{C}_{40} \mathrm{H}_{59} \mathrm{NO}_{4} \mathrm{Si}_{2} \mathrm{Na}(\mathrm{M}+\mathrm{Na})^{+}$696.3881, found 696.3884. Anal. Calcd for $\mathrm{C}_{40} \mathrm{H}_{59} \mathrm{NO}_{4} \mathrm{Si}_{2}: \mathrm{C}, 71.27 ; \mathrm{H}, 8.82 ; \mathrm{N}, 2.08$. Found: C, 71.16; H, 8.93; N, 2.04 . 


\section{Regiochemistry Assignment of Oxasilacyclopentenes by NOE}

The regiochemistry was assigned using DPFSG-NOE experiments, X-ray crystallography, and by analogy with ${ }^{1} \mathrm{H}$ NMR spectroscopy as compared to similar substrates determined by the previous methods. In several cases, the minor regioisomer could be isolated in sufficient quantity to provide NOE's of both isomers. A summary of the aforementioned data follows:

\section{NOE Experiments:}
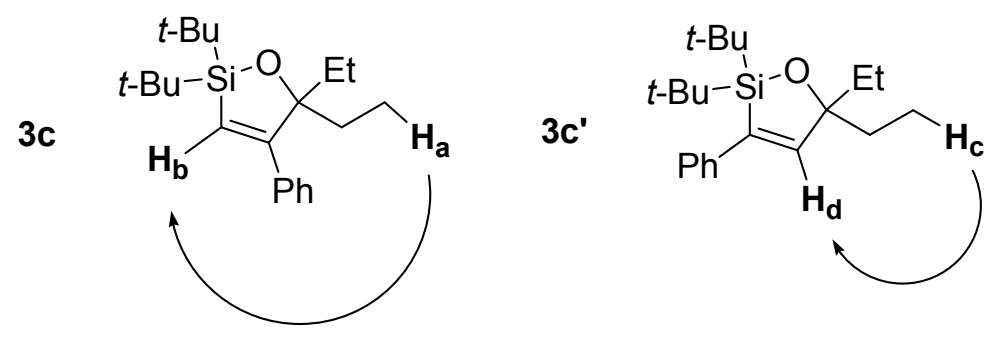

2,2-Di-tert-butyl-4-phenyl-5,5-diethyloxasilacyclopentene (3c)

\section{Major Isomer:}

$\mathbf{H}_{\mathbf{b}}: \delta 5.70 \mathrm{ppm}$

$\mathbf{H}_{\mathbf{a}}$ irradiated: No NOE observed to $\mathbf{H}_{\mathbf{b}}$

2,2-Di-tert-butyl-3-phenyl-5,5-diethyloxasilacyclopentene (3c')

\section{Minor Isomer:}

$\mathbf{H}_{\mathbf{d}}: \delta 7.02 \mathrm{ppm}$

$\mathbf{H}_{\mathbf{c}}$ irradiated: $1.8 \%$ NOE observed to $\mathbf{H}_{\mathbf{d}}$
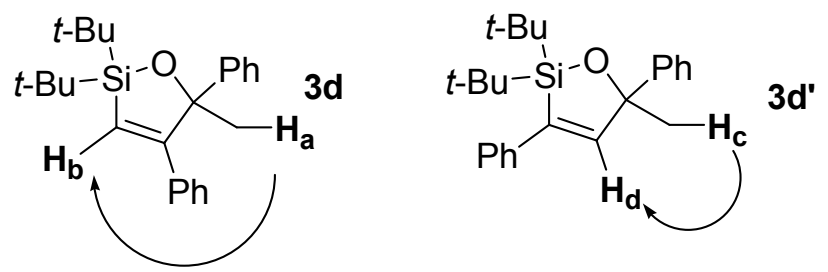

2,2-Di-tert-butyl-4,5-diphenyl-5-methyloxasilacyclopentene (3d)

\section{Major Isomer:}

$\mathbf{H}_{\mathbf{b}}: \delta 6.44 \mathrm{ppm}$

$\mathbf{H}_{\mathbf{a}}$ irradiated: No NOE observed to $\mathbf{H}_{\mathrm{b}}$

2,2-Di-tert-butyl-3,5-diphenyl-5-methyloxasilacyclopentene (3d')

\section{Minor Isomer:}

$\mathbf{H}_{\mathbf{d}}: \delta 7.37 \mathrm{ppm}$

$\mathbf{H}_{\mathbf{c}}$ irradiated: $6.8 \%$ NOE observed to $\mathbf{H}_{\mathbf{d}}$ 


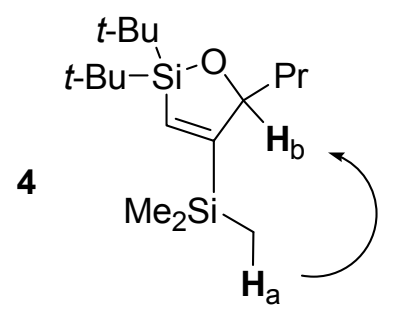

2,2-Di-tert-butyl-4-trimethylsilyl-5-propyloxasilacyclopentene (4)

$\mathbf{H}_{\mathbf{a}}$ irradiated: $4.2 \%$ NOE observed to $\mathbf{H}_{\mathbf{b}}$

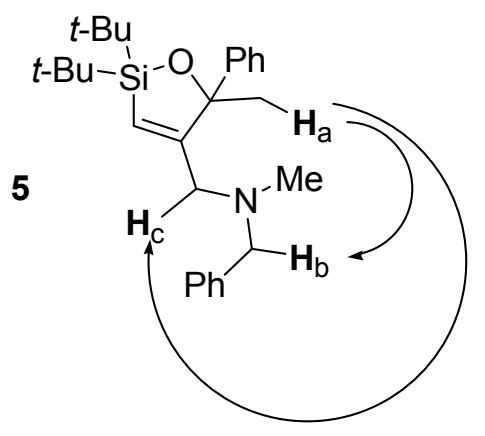

$N$-Benzyl- $N$-(2,2-Di-tert-butyl-5-phenyl-5-methyl-4-methyloxasilacyclopentenyl)methylamine (5)

$\mathbf{H}_{\mathbf{a}}$ irradiated: $9.6 \%$ NOE observed to $\mathbf{H}_{\mathbf{b}}, 12.1 \%$ NOE observed to $\mathbf{H}_{\mathbf{c}}$

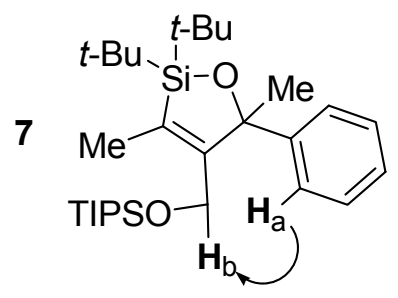

2,2-Di-tert-butyl-3-methyl-4-triisopropylsiloxymethyl-5-methyl-5-phenyloxasilacyclopentene (7)

$\mathbf{H}_{\mathbf{a}}$ irradiated: $3.0 \%$ NOE observed to $\mathbf{H}_{\mathbf{b}}$ 
Major Regioisomer Determined by Analogy: ${ }^{1} \mathrm{H}$ NMR spectroscopic analysis of substrates $\mathbf{3 c}$ and $\mathbf{3 d}$ display a large difference between the chemical shift of the vinyl hydrogens for each regioisomer. Several determinations of major regioisomers were carried out by analogy to this data.

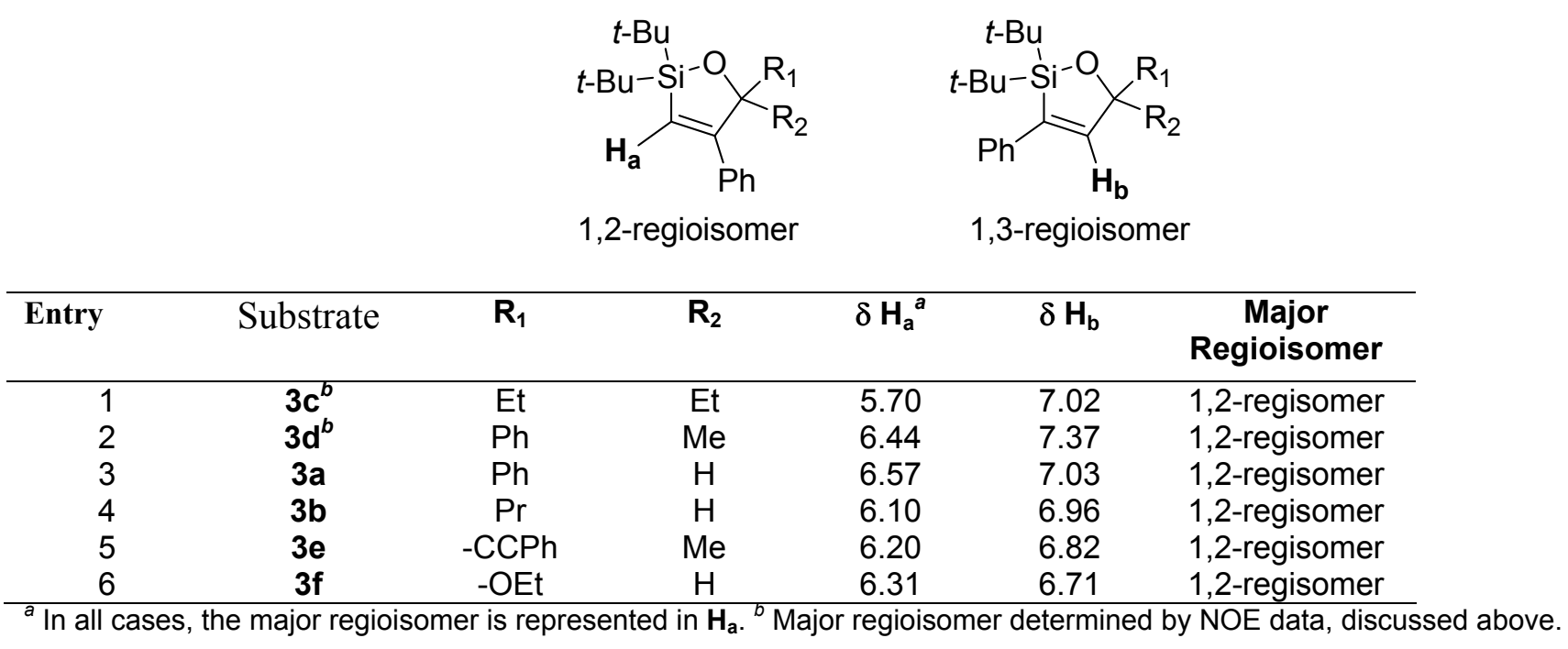

The relative stereochemistry of diol $\mathbf{9}$ was established by NOE experiments and coupling constants of acetal $\mathbf{B}$.

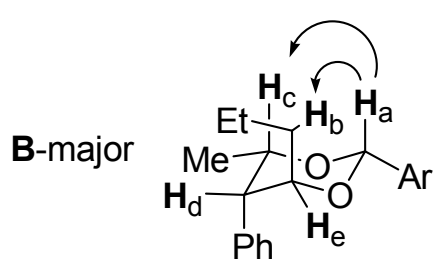

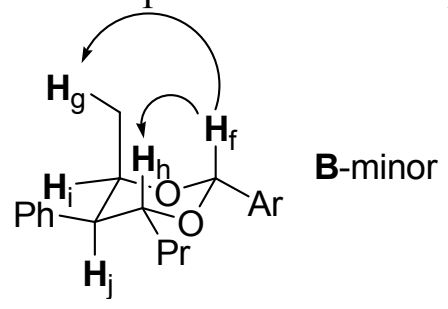

$\left(2 R^{*}, 4 R^{*}, 5 S^{*}, 6 R^{*}\right)-4-$ Methyl-2-(nitrophenyl)-5-phenyl-6-propyl-1,3-dioxane (B)

B-Major:

$\mathbf{H}_{\mathbf{a}}$ irradiated: $3.6 \%$ NOE observed to $\mathbf{H}_{\mathrm{b}}, 14.0 \%$ NOE observed to $\mathbf{H}_{\mathbf{c}}$

$\mathbf{H}_{\mathbf{d}}$ is a d, $J_{H c}$ to $H d=3.1 \mathrm{~Hz}, J_{\text {He to } H d}=$ ca. $0 \mathrm{~Hz}$

B-Minor:

$\mathbf{H}_{\mathbf{f}}$ irradiated: $2.9 \%$ NOE observed to $\mathbf{H}_{\mathbf{g}}, \mathrm{NOE}$ to $\mathbf{H}_{\mathbf{h}}$ is present, however $\delta$ of $\mathbf{H}_{\mathbf{h}}$ overlaps with $\mathbf{H}_{\mathrm{i}}$.

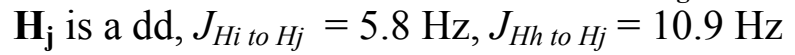




\section{References}

(1) Trost, B. M.; Imi, K.; Indolese, A. F. J. Am. Chem. Soc. 1993, 115, 8831-8832.

(2) Driver, T. G.; Franz, A. K.; Woerpel, K. A. J. Am. Chem. Soc. 2002, 124, 6524-6525.

(3) Boudjouk, P.; Samaraweera, U.; Sooriyakumaran, R.; Chrusciel, J.; Anderson, K. R. Angew. Chem., Int. Ed. Engl. 1988, 100, 1406-1407.

(4) Tsai, D. J. S.; Midland, M. M. J. Am. Chem. Soc. 1985, 107, 3915-3918.

(5) Palmer, W. S.; Woerpel, K. A. Organometallics 1997, 16, 4824-4827. 


\section{X-Ray Crystallography Data:}

X-ray Data Collection, Structure Solution and Refinement for $6 \mathbf{a}$.

A colorless crystal of approximate dimensions $0.14 \times 0.22 \times 0.24 \mathrm{~mm}$ was mounted on a glass fiber and transferred to a Bruker CCD platform diffractometer. The SMART ${ }^{1}$ program package was used to determine the unit-cell parameters and for data collection $(25 \mathrm{sec} /$ frame scan time for a sphere of diffraction data). The raw frame data was processed using $\mathrm{SAINT}^{2}$ and $\mathrm{SADABS}^{3}$ to yield the reflection data file. Subsequent calculations were carried out using the SHELXTL ${ }^{4}$ program. The diffraction symmetry was $2 / m$ and the systematic absences were consistent with the centrosymmetric monoclinic space group $P 2_{1} / c$ which was later determined to be correct.

The structure was solved by direct methods and refined on $\mathrm{F}^{2}$ by full-matrix least-squares techniques. The analytical scattering factors 5 for neutral atoms were used throughout the analysis. Hydrogen atoms were included using a riding model. There were two molecules of the formula unit present. The space group was monoclinic with beta approximately $90^{\circ}$ (emulates orthorhombic crystal system). It was necessary to treat the structure as a twin and refine using the TWIN command. At convergence, $w R 2=0.2072$ and Goof $=1.029$ for 489 variables refined against 7669 data $(\AA)$. As a comparison for refinement on $F, R 1=0.0713$ for those 4778 data with $\mathrm{I}>2.0 \sigma(\mathrm{I})$.

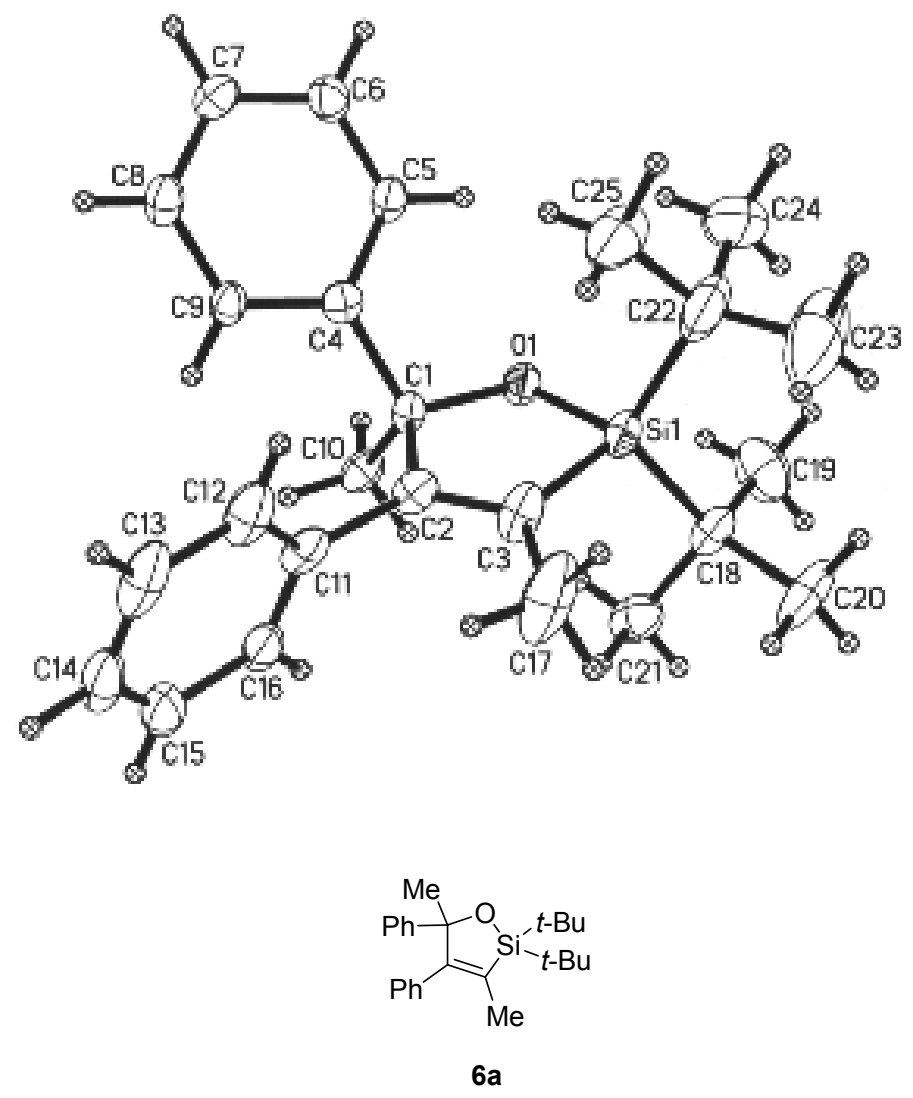




\section{References.}

1. SMART Software Users Guide, Version 5.1, Bruker Analytical X-Ray Systems, Inc.; Madison, WI 1999.

2. SAINT Software Users Guide, Version 6.0, Bruker Analytical X-Ray Systems, Inc.; Madison, WI 1999.

3. Sheldrick, G. M. SADABS, Version 2.05, Bruker Analytical X-Ray Systems, Inc.; Madison, WI 2001.

4. Sheldrick, G. M. SHELXTL Version 6.12, Bruker Analytical X-Ray Systems, Inc.; Madison, WI 2001.

5. International Tables for X-Ray Crystallography 1992, Vol. C., Dordrecht: Kluwer AcademicPublishers.

\section{Definitions:}

$\mathrm{wR} 2=\left[\Sigma\left[\mathrm{w}\left(\mathrm{F}_{\mathrm{o}}^{2}-\mathrm{F}_{\mathrm{c}}\right)^{2}\right] / \Sigma\left[\mathrm{w}\left(\mathrm{F}_{\mathrm{o}}{ }^{2}\right)^{2}\right]^{1 / 2}\right.$

$\mathrm{R} 1=\Sigma|| \mathrm{F}_{\mathrm{o}}|-| \mathrm{F}_{\mathrm{c}}|| / \Sigma\left|\mathrm{F}_{\mathrm{o}}\right|$

Goof $=\mathrm{S}=\left[\Sigma\left[\mathrm{w}\left(\mathrm{F}_{\mathrm{o}}^{2}-\mathrm{F}_{\mathrm{c}}{ }^{2}\right)^{2}\right] /(\mathrm{n}-\mathrm{p})\right]^{1 / 2}$ where $\mathrm{n}$ is the number of reflections and $\mathrm{p}$ is the total number of parameters refined.

The thermal ellipsoid plot is shown at the $40 \%$ probability level.

Table 1. Crystal data and structure refinement for $\mathbf{6 a}$.

Identification code

Empirical formula

Formula weight

Temperature

Wavelength

Crystal system

Space group

Unit cell dimensions

Volume

Z kaw42

$\mathrm{C}_{25} \mathrm{H}_{34} \mathrm{O} \mathrm{Si}$

378.61

163(2) K

$0.71073 \AA$

Monoclinic

$P 2{ }_{1} / c$

$\mathrm{a}=14.6527(7) \AA \quad \alpha=90^{\circ}$.

$\mathrm{b}=41.0065(19) \AA \quad \beta=90.0080(10)^{\circ}$.

$\mathrm{c}=7.4830(3) \AA \quad \gamma=90^{\circ}$.

4496.2(3) $\AA^{3}$

8 
Density (calculated)

Absorption coefficient

$\mathrm{F}(000)$

Crystal size

Theta range for data collection

Index ranges

Reflections collected

Independent reflections

Completeness to theta $=24.71^{\circ}$

Absorption correction

Max. and min. transmission

Refinement method

Data / restraints / parameters

Goodness-of-fit on $\mathrm{F}^{2}$

Final $\mathrm{R}$ indices $[\mathrm{I}>2 \operatorname{sigma}(\mathrm{I})]$

$\mathrm{R}$ indices (all data; $0.85 \AA$ )

Extinction coefficient

Largest diff. peak and hole
$1.119 \mathrm{Mg} / \mathrm{m}^{3}$

$0.116 \mathrm{~mm}^{-1}$

1648

$0.24 \times 0.22 \times 0.14 \mathrm{~mm}^{3}$

1.39 to $24.71^{\circ}$.

$-17 \leq h \leq 17,-48 \leq k \leq 48,-8 \leq l \leq 8$

39061

$7669[\mathrm{R}(\mathrm{int})=0.0998]$

$100.0 \%$

Semi-empirical from equivalents

0.9840 and 0.9727

Full-matrix least-squares on $\mathrm{F}^{2}$

7669 / 0 / 489

1.029

$\mathrm{R} 1=0.0713, \mathrm{wR} 2=0.1695$

$\mathrm{R} 1=0.1304, \mathrm{wR} 2=0.2072$

$0.0012(5)$

0.775 and -0.612 e. $\AA^{-3}$ 
Table 2. Atomic coordinates $\left(\mathrm{x} 10^{4}\right)$ and equivalent isotropic displacement parameters $\left(\AA^{2} \times 10^{3}\right)$ for 6a. $U(e q)$ is defined as one third of the trace of the orthogonalized $U^{i j}$ tensor.

\begin{tabular}{lrrrr}
\hline & $\mathrm{x}$ & $\mathrm{y}$ & $\mathrm{z}$ & $\mathrm{U}(\mathrm{eq})$ \\
\hline $\mathrm{Si}(1)$ & & & & \\
$\mathrm{O}(1)$ & $9658(1)$ & $859(1)$ & $7317(2)$ & $36(1)$ \\
$\mathrm{C}(1)$ & $9643(3)$ & $1147(1)$ & $5761(5)$ & $34(1)$ \\
$\mathrm{C}(2)$ & $9524(3)$ & $1476(1)$ & $6409(7)$ & $26(1)$ \\
$\mathrm{C}(3)$ & $9404(4)$ & $1446(1)$ & $8461(7)$ & $33(1)$ \\
$\mathrm{C}(4)$ & $9471(4)$ & $1148(1)$ & $9169(8)$ & $42(2)$ \\
$\mathrm{C}(5)$ & $10362(3)$ & $1678(1)$ & $5859(7)$ & $29(1)$ \\
$\mathrm{C}(6)$ & $11008(4)$ & $1553(1)$ & $4690(7)$ & $32(1)$ \\
$\mathrm{C}(7)$ & $11737(4)$ & $1737(1)$ & $4120(8)$ & $38(1)$ \\
$\mathrm{C}(8)$ & $11838(4)$ & $2057(1)$ & $4686(8)$ & $37(1)$ \\
$\mathrm{C}(9)$ & $11195(4)$ & $2189(1)$ & $5821(8)$ & $38(1)$ \\
$\mathrm{C}(10)$ & $10454(4)$ & $2001(1)$ & $6412(7)$ & $30(1)$ \\
$\mathrm{C}(11)$ & $8672(4)$ & $1617(1)$ & $5497(7)$ & $35(1)$ \\
$\mathrm{C}(12)$ & $9179(4)$ & $1742(1)$ & $9557(7)$ & $35(1)$ \\
$\mathrm{C}(13)$ & $9842(5)$ & $1880(2)$ & $10674(8)$ & $50(2)$ \\
$\mathrm{C}(14)$ & $9608(7)$ & $2140(2)$ & $11797(8)$ & $65(2)$ \\
$\mathrm{C}(15)$ & $8719(7)$ & $2258(2)$ & $11818(10)$ & $69(2)$ \\
$\mathrm{C}(16)$ & $8073(5)$ & $2122(2)$ & $10722(10)$ & $62(2)$ \\
$\mathrm{C}(17)$ & $8299(5)$ & $1859(1)$ & $9604(8)$ & $46(2)$ \\
$\mathrm{C}(18)$ & $9364(6)$ & $1074(2)$ & $11135(9)$ & $81(3)$ \\
$\mathrm{C}(19)$ & $8671(4)$ & $570(1)$ & $6935(8)$ & $40(2)$ \\
$\mathrm{C}(20)$ & $8718(5)$ & $400(2)$ & $5130(10)$ & $67(2)$ \\
$\mathrm{C}(21)$ & $8584(5)$ & $318(2)$ & $8438(10)$ & $70(2)$ \\
$\mathrm{C}(22)$ & $7790(4)$ & $777(2)$ & $6935(8)$ & $49(2)$ \\
$\mathrm{C}(23)$ & $10820(5)$ & $653(2)$ & $7322(16)$ & $92(3)$ \\
$\mathrm{C}(24)$ & $10888(6)$ & $363(2)$ & $8430(20)$ & $176(7)$ \\
$\mathrm{C}(25)$ & $11048(6)$ & $547(2)$ & $5219(14)$ & $104(4)$ \\
$\mathrm{Si}(2)$ & $11529(4)$ & $917(2)$ & $7855(13)$ & $79(3)$ \\
$\mathrm{O}(2)$ & $4869(1)$ & $865(1)$ & $2304(2)$ & $35(1)$ \\
$\mathrm{C}(31)$ & $4877(2)$ & $1153(1)$ & $750(5)$ & $32(1)$ \\
& $4980(3)$ & $1480(1)$ & $1410(7)$ & $29(1)$ \\
& & & &
\end{tabular}




$\begin{array}{lrrrr}\mathrm{C}(32) & 5112(4) & 1455(1) & 3453(7) & 31(1) \\ \mathrm{C}(33) & 5059(4) & 1155(1) & 4145(7) & 39(2) \\ \mathrm{C}(34) & 4156(3) & 1683(1) & 856(7) & 29(1) \\ \mathrm{C}(35) & 3505(4) & 1553(1) & -331(7) & 34(1) \\ \mathrm{C}(36) & 2773(4) & 1743(1) & -909(8) & 42(1) \\ \mathrm{C}(37) & 2669(4) & 2057(1) & -323(7) & 38(1) \\ \mathrm{C}(38) & 3300(4) & 2192(1) & 850(8) & 37(1) \\ \mathrm{C}(39) & 4038(4) & 2003(1) & 1401(7) & 34(1) \\ \mathrm{C}(40) & 1628(1) & 509(7) & 36(1) \\ \mathrm{C}(41) & 5825(4) & 1746(1) & 4555(7) & 37(1) \\ \mathrm{C}(42) & 5333(4) & 1887(1) & 5676(8) & 45(2) \\ \mathrm{C}(43) & 4684(5) & 2144(2) & 6798(8) & 59(2) \\ \mathrm{C}(44) & 4909(6) & 6823(10) & 68(2) \\ \mathrm{C}(45) & 5779(7) & 2262(2) & 682) \\ \mathrm{C}(46) & 6452(5) & 2128(2) & 5753(10) & 59(2) \\ \mathrm{C}(47) & 1868(1) & 4638(9) & 46(2) \\ \mathrm{C}(48) & 6222(4) & 1080(2) & 6122(8) & 74(2) \\ \mathrm{C}(49) & 582(1) & 1908(7) & 37(1) \\ \mathrm{C}(50) & 3186(6) & 307(2) & 3266(10) & 66(2) \\ \mathrm{C}(51) & 5871(4) & 534(2) & 11(8) & 54(2) \\ \mathrm{C}(52) & 787(1) & 1981(8) & 47(2) \\ \mathrm{C}(53) & 659(2) & 2375(14) & 79(3) \\ \mathrm{C}(54) & 5941(5) & 550(2) & 164(7) \\ & 5814(5) & 921(2) & 2873(12) & 70(2)\end{array}$

Table 3. Bond lengths $[\AA]$ and angles $\left[^{\circ}\right]$ for $\mathbf{6 a}$.

\begin{tabular}{ll}
\hline $\mathrm{Si}(1)-\mathrm{O}(1)$ & $1.658(4)$ \\
$\mathrm{Si}(1)-\mathrm{C}(3)$ & $1.843(6)$ \\
$\mathrm{Si}(1)-\mathrm{C}(18)$ & $1.891(6)$ \\
$\mathrm{Si}(1)-\mathrm{C}(22)$ & $1.901(8)$ \\
$\mathrm{O}(1)-\mathrm{C}(1)$ & $1.447(6)$ \\
$\mathrm{C}(1)-\mathrm{C}(10)$ & $1.534(7)$ \\
$\mathrm{C}(1)-\mathrm{C}(4)$ & $1.537(7)$ \\
$\mathrm{C}(1)-\mathrm{C}(2)$ & $1.551(7)$
\end{tabular}




$\begin{array}{ll}\mathrm{C}(2)-\mathrm{C}(3) & 1.338(8) \\ \mathrm{C}(2)-\mathrm{C}(11) & 1.500(8) \\ \mathrm{C}(3)-\mathrm{C}(17) & 1.510(9) \\ \mathrm{C}(4)-\mathrm{C}(5) & 1.387(7) \\ \mathrm{C}(4)-\mathrm{C}(9) & 1.393(7) \\ \mathrm{C}(5)-\mathrm{C}(6) & 1.375(7) \\ \mathrm{C}(6)-\mathrm{C}(7) & 1.386(7) \\ \mathrm{C}(7)-\mathrm{C}(8) & 1.380(8) \\ \mathrm{C}(8)-\mathrm{C}(9) & 1.403(7) \\ \mathrm{C}(11)-\mathrm{C}(16) & 1.377(8) \\ \mathrm{C}(11)-\mathrm{C}(12) & 1.402(8) \\ \mathrm{C}(12)-\mathrm{C}(13) & 1.399(9) \\ \mathrm{C}(13)-\mathrm{C}(14) & 1.390(10) \\ \mathrm{C}(14)-\mathrm{C}(15) & 1.371(10) \\ \mathrm{C}(15)-\mathrm{C}(16) & 1.402(8) \\ \mathrm{C}(18)-\mathrm{C}(19) & 1.522(9) \\ \mathrm{C}(18)-\mathrm{C}(20) & 1.534(8) \\ \mathrm{C}(18)-\mathrm{C}(21) & 1.547(8) \\ \mathrm{C}(22)-\mathrm{C}(23) & 1.453(12) \\ \mathrm{C}(22)-\mathrm{C}(25) & 1.552(10) \\ \mathrm{C}(22)-\mathrm{C}(24) & 1.667(14) \\ \mathrm{Si}(2)-\mathrm{O}(2) & 1.658(4) \\ \mathrm{Si}(2)-\mathrm{C}(33) & 1.841(6) \\ \mathrm{Si}(2)-\mathrm{C}(48) & 1.893(6) \\ \mathrm{Si}(2)-\mathrm{C}(52) & 1.902(7) \\ \mathrm{O}(2)-\mathrm{C}(31) & 1.436(6) \\ \mathrm{C}(31)-\mathrm{C}(34) & 1.523(7) \\ \mathrm{C}(31)-\mathrm{C}(40) & 1.534(7) \\ \mathrm{C}(31)-\mathrm{C}(32) & 1.544(7) \\ \mathrm{C}(32)-\mathrm{C}(33) & 1.339(7) \\ \mathrm{C}(32)-\mathrm{C}(41) & 1.486(8) \\ \mathrm{C}(33)-\mathrm{C}(47) & 1.521(8) \\ \mathrm{C}(34)-\mathrm{C}(39) & 1.386(7) \\ \mathrm{C}(34)-\mathrm{C}(35) & 1.408(7) \\ \mathrm{C}(35)-\mathrm{C}(36) & \\ & \end{array}$




$\begin{array}{ll}\mathrm{C}(36)-\mathrm{C}(37) & 1.372(8) \\ \mathrm{C}(37)-\mathrm{C}(38) & 1.389(8) \\ \mathrm{C}(38)-\mathrm{C}(39) & 1.392(7) \\ \mathrm{C}(41)-\mathrm{C}(42) & 1.393(8) \\ \mathrm{C}(41)-\mathrm{C}(46) & 1.397(8) \\ \mathrm{C}(42)-\mathrm{C}(43) & 1.387(9) \\ \mathrm{C}(43)-\mathrm{C}(44) & 1.364(10) \\ \mathrm{C}(44)-\mathrm{C}(45) & 1.384(11) \\ \mathrm{C}(45)-\mathrm{C}(46) & 1.394(8) \\ \mathrm{C}(48)-\mathrm{C}(51) & 1.523(8) \\ \mathrm{C}(48)-\mathrm{C}(49) & 1.524(8) \\ \mathrm{C}(48)-\mathrm{C}(50) & 1.546(8) \\ \mathrm{C}(52)-\mathrm{C}(54) & 1.517(11) \\ \mathrm{C}(52)-\mathrm{C}(55) & 1.526(9) \\ \mathrm{C}(52)-\mathrm{C}(53) & 1.531(15)\end{array}$

$\begin{array}{lr}\mathrm{O}(1)-\mathrm{Si}(1)-\mathrm{C}(3) & 94.0(2) \\ \mathrm{O}(1)-\mathrm{Si}(1)-\mathrm{C}(18) & 109.3(2) \\ \mathrm{C}(3)-\mathrm{Si}(1)-\mathrm{C}(18) & 113.7(3) \\ \mathrm{O}(1)-\mathrm{Si}(1)-\mathrm{C}(22) & 109.2(3) \\ \mathrm{C}(3)-\mathrm{Si}(1)-\mathrm{C}(22) & 114.7(4) \\ \mathrm{C}(18)-\mathrm{Si}(1)-\mathrm{C}(22) & 114.0(3) \\ \mathrm{C}(1)-\mathrm{O}(1)-\mathrm{Si}(1) & 115.6(3) \\ \mathrm{O}(1)-\mathrm{C}(1)-\mathrm{C}(10) & 107.4(4) \\ \mathrm{O}(1)-\mathrm{C}(1)-\mathrm{C}(4) & 108.4(4) \\ \mathrm{C}(10)-\mathrm{C}(1)-\mathrm{C}(4) & 109.3(4) \\ \mathrm{O}(1)-\mathrm{C}(1)-\mathrm{C}(2) & 105.7(4) \\ \mathrm{C}(10)-\mathrm{C}(1)-\mathrm{C}(2) & 112.2(4) \\ \mathrm{C}(4)-\mathrm{C}(1)-\mathrm{C}(2) & 113.5(4) \\ \mathrm{C}(3)-\mathrm{C}(2)-\mathrm{C}(11) & 122.6(5) \\ \mathrm{C}(3)-\mathrm{C}(2)-\mathrm{C}(1) & 117.2(5) \\ \mathrm{C}(11)-\mathrm{C}(2)-\mathrm{C}(1) & 120.1(5) \\ \mathrm{C}(2)-\mathrm{C}(3)-\mathrm{C}(17) & 124.1(6) \\ \mathrm{C}(2)-\mathrm{C}(3)-\mathrm{Si}(1) & 107.5(4) \\ \mathrm{C}(17)-\mathrm{C}(3)-\mathrm{Si}(1) & 128.3(5)\end{array}$




$\begin{array}{lr}\mathrm{C}(5)-\mathrm{C}(4)-\mathrm{C}(9) & 118.2(5) \\ \mathrm{C}(5)-\mathrm{C}(4)-\mathrm{C}(1) & 121.0(5) \\ \mathrm{C}(9)-\mathrm{C}(4)-\mathrm{C}(1) & 120.6(5) \\ \mathrm{C}(6)-\mathrm{C}(5)-\mathrm{C}(4) & 121.5(5) \\ \mathrm{C}(5)-\mathrm{C}(6)-\mathrm{C}(7) & 120.5(5) \\ \mathrm{C}(8)-\mathrm{C}(7)-\mathrm{C}(6) & 119.1(5) \\ \mathrm{C}(7)-\mathrm{C}(8)-\mathrm{C}(9) & 120.5(5) \\ \mathrm{C}(4)-\mathrm{C}(9)-\mathrm{C}(8) & 120.2(5) \\ \mathrm{C}(16)-\mathrm{C}(11)-\mathrm{C}(12) & 119.5(6) \\ \mathrm{C}(16)-\mathrm{C}(11)-\mathrm{C}(2) & 120.2(5) \\ \mathrm{C}(12)-\mathrm{C}(11)-\mathrm{C}(2) & 120.0(6) \\ \mathrm{C}(13)-\mathrm{C}(12)-\mathrm{C}(11) & 119.7(7) \\ \mathrm{C}(14)-\mathrm{C}(13)-\mathrm{C}(12) & 120.1(7) \\ \mathrm{C}(15)-\mathrm{C}(14)-\mathrm{C}(13) & 119.9(7) \\ \mathrm{C}(14)-\mathrm{C}(15)-\mathrm{C}(16) & 120.4(7) \\ \mathrm{C}(11)-\mathrm{C}(16)-\mathrm{C}(15) & 120.3(6) \\ \mathrm{C}(19)-\mathrm{C}(18)-\mathrm{C}(20) & 110.2(5) \\ \mathrm{C}(19)-\mathrm{C}(18)-\mathrm{C}(21) & 106.9(5) \\ \mathrm{C}(20)-\mathrm{C}(18)-\mathrm{C}(21) & 107.5(5) \\ \mathrm{C}(19)-\mathrm{C}(18)-\mathrm{Si}(1) & 112.8(4) \\ \mathrm{C}(20)-\mathrm{C}(18)-\mathrm{Si}(1) & 112.0(4) \\ \mathrm{C}(21)-\mathrm{C}(18)-\mathrm{Si}(1) & 107.1(4) \\ \mathrm{C}(23)-\mathrm{C}(22)-\mathrm{C}(25) & 112.1(8) \\ \mathrm{C}(23)-\mathrm{C}(22)-\mathrm{C}(24) & 108.2(9) \\ \mathrm{C}(25)-\mathrm{C}(22)-\mathrm{C}(24) & 106.9(7) \\ \mathrm{C}(23)-\mathrm{C}(22)-\mathrm{Si}(1) & 115.2(6) \\ \mathrm{C}(25)-\mathrm{C}(22)-\mathrm{Si}(1) & 106.9(5) \\ \mathrm{C}(24)-\mathrm{C}(22)-\mathrm{Si}(1) & 107.1(6) \\ \mathrm{O}(2)-\mathrm{Si}(2)-\mathrm{C}(33) & 93.6(2) \\ \mathrm{O}(2)-\mathrm{Si}(2)-\mathrm{C}(48) & 108.7(2) \\ \mathrm{C}(33)-\mathrm{Si}(2)-\mathrm{C}(48) & 113.3(3) \\ \mathrm{O}(2)-\mathrm{Si}(2)-\mathrm{C}(52) & 110.1(3) \\ \mathrm{C}(33)-\mathrm{Si}(2)-\mathrm{C}(52) & 113.6(4) \\ \mathrm{C}(48)-\mathrm{Si}(2)-\mathrm{C}(52) & 115.3(3) \\ \mathrm{C}(31)-\mathrm{O}(2)-\mathrm{Si}(2) & 115.2(3)\end{array}$




$\begin{array}{ll}\mathrm{O}(2)-\mathrm{C}(31)-\mathrm{C}(34) & 109.4(4) \\ \mathrm{O}(2)-\mathrm{C}(31)-\mathrm{C}(40) & 107.6(4) \\ \mathrm{C}(34)-\mathrm{C}(31)-\mathrm{C}(40) & 107.7(4) \\ \mathrm{O}(2)-\mathrm{C}(31)-\mathrm{C}(32) & 107.0(4) \\ \mathrm{C}(34)-\mathrm{C}(31)-\mathrm{C}(32) & 113.9(4) \\ \mathrm{C}(40)-\mathrm{C}(31)-\mathrm{C}(32) & 111.1(5) \\ \mathrm{C}(33)-\mathrm{C}(32)-\mathrm{C}(41) & 122.5(5) \\ \mathrm{C}(33)-\mathrm{C}(32)-\mathrm{C}(31) & 115.9(5) \\ \mathrm{C}(41)-\mathrm{C}(32)-\mathrm{C}(31) & 121.6(5) \\ \mathrm{C}(32)-\mathrm{C}(33)-\mathrm{C}(47) & 123.6(5) \\ \mathrm{C}(32)-\mathrm{C}(33)-\mathrm{Si}(2) & 108.3(4) \\ \mathrm{C}(47)-\mathrm{C}(33)-\mathrm{Si}(2) & 128.1(4) \\ \mathrm{C}(39)-\mathrm{C}(34)-\mathrm{C}(35) & 117.2(5) \\ \mathrm{C}(39)-\mathrm{C}(34)-\mathrm{C}(31) & 122.4(5) \\ \mathrm{C}(35)-\mathrm{C}(34)-\mathrm{C}(31) & 120.3(5) \\ \mathrm{C}(36)-\mathrm{C}(35)-\mathrm{C}(34) & 120.5(5) \\ \mathrm{C}(37)-\mathrm{C}(36)-\mathrm{C}(35) & 120.7(5) \\ \mathrm{C}(36)-\mathrm{C}(37)-\mathrm{C}(38) & 120.1(5) \\ \mathrm{C}(37)-\mathrm{C}(38)-\mathrm{C}(39) & 118.9(5) \\ \mathrm{C}(34)-\mathrm{C}(39)-\mathrm{C}(38) & 122.5(5) \\ \mathrm{C}(42)-\mathrm{C}(41)-\mathrm{C}(46) & 117.4(6) \\ \mathrm{C}(42)-\mathrm{C}(41)-\mathrm{C}(32) & 121.3(6) \\ \mathrm{C}(46)-\mathrm{C}(41)-\mathrm{C}(32) & 121.0(5) \\ \mathrm{C}(43)-\mathrm{C}(42)-\mathrm{C}(41) & 121.2(7) \\ \mathrm{C}(44)-\mathrm{C}(43)-\mathrm{C}(42) & 120.0(6) \\ \mathrm{C}(43)-\mathrm{C}(44)-\mathrm{C}(45) & 121.1(7) \\ \mathrm{C}(44)-\mathrm{C}(45)-\mathrm{C}(46) & 118.6(7) \\ \mathrm{C}(45)-\mathrm{C}(46)-\mathrm{C}(41) & 121.7(6) \\ \mathrm{C}(51)-\mathrm{C}(48)-\mathrm{C}(49) & 109.2(5) \\ \mathrm{C}(51)-\mathrm{C}(48)-\mathrm{C}(50) & 107.1(5) \\ \mathrm{C}(49)-\mathrm{C}(48)-\mathrm{C}(50) & 108.9(5) \\ \mathrm{C}(51)-\mathrm{C}(48)-\mathrm{Si}(2) & 107.7(4) \\ \mathrm{C}(49)-\mathrm{C}(48)-\mathrm{Si}(2) & 113.7(4) \\ \mathrm{C}(50)-\mathrm{C}(48)-\mathrm{Si}(2) & 110.0(4) \\ \mathrm{C}(54)-\mathrm{C}(52)-\mathrm{C}(55) & 110.1(8)\end{array}$




$\begin{array}{ll}\mathrm{C}(54)-\mathrm{C}(52)-\mathrm{C}(53) & 108.1(9) \\ \mathrm{C}(55)-\mathrm{C}(52)-\mathrm{C}(53) & 108.8(7) \\ \mathrm{C}(54)-\mathrm{C}(52)-\mathrm{Si}(2) & 111.8(6) \\ \mathrm{C}(55)-\mathrm{C}(52)-\mathrm{Si}(2) & 107.0(5) \\ \mathrm{C}(53)-\mathrm{C}(52)-\mathrm{Si}(2) & 111.1(7)\end{array}$

Table 4. Anisotropic displacement parameters $\left(\AA^{2} \times 10^{3}\right)$ for $\mathbf{6 a}$. The anisotropic displacement factor exponent takes the form: $-2 \pi^{2}\left[h^{2} a^{* 2} U^{11}+\ldots+2 h k a^{*} b^{*} U^{12}\right]$

\begin{tabular}{lcccccc}
\hline & $\mathrm{U}^{11}$ & $\mathrm{U}^{22}$ & $\mathrm{U}^{33}$ & $\mathrm{U}^{23}$ & $\mathrm{U}^{13}$ & $\mathrm{U}^{12}$ \\
\hline $\mathrm{Si}(1)$ & $41(1)$ & $26(1)$ & $41(1)$ & $6(1)$ & $0(1)$ & $-6(1)$ \\
$\mathrm{O}(1)$ & $47(2)$ & $28(2)$ & $27(2)$ & $-2(2)$ & $9(2)$ & $-4(2)$ \\
$\mathrm{C}(1)$ & $33(3)$ & $21(3)$ & $24(3)$ & $0(2)$ & $-1(2)$ & $-3(2)$ \\
$\mathrm{C}(2)$ & $38(3)$ & $38(3)$ & $22(3)$ & $-2(2)$ & $-3(2)$ & $-10(3)$ \\
$\mathrm{C}(3)$ & $59(4)$ & $35(3)$ & $32(3)$ & $5(3)$ & $-13(3)$ & $-14(3)$ \\
$\mathrm{C}(4)$ & $38(3)$ & $31(3)$ & $17(3)$ & $5(2)$ & $0(2)$ & $1(2)$ \\
$\mathrm{C}(5)$ & $42(3)$ & $24(3)$ & $29(3)$ & $1(2)$ & $2(3)$ & $-2(2)$ \\
$\mathrm{C}(6)$ & $44(3)$ & $33(3)$ & $37(3)$ & $3(3)$ & $7(3)$ & $3(3)$ \\
$\mathrm{C}(7)$ & $38(3)$ & $38(3)$ & $36(3)$ & $4(3)$ & $3(3)$ & $-8(3)$ \\
$\mathrm{C}(8)$ & $51(4)$ & $29(3)$ & $34(3)$ & $3(3)$ & $0(3)$ & $-3(3)$ \\
$\mathrm{C}(9)$ & $42(3)$ & $24(3)$ & $24(3)$ & $1(2)$ & $4(2)$ & $-4(2)$ \\
$\mathrm{C}(10)$ & $38(3)$ & $37(3)$ & $29(3)$ & $9(3)$ & $-4(2)$ & $-9(2)$ \\
$\mathrm{C}(11)$ & $52(4)$ & $39(3)$ & $14(3)$ & $2(3)$ & $3(3)$ & $-17(3)$ \\
$\mathrm{C}(12)$ & $81(5)$ & $41(4)$ & $26(3)$ & $4(3)$ & $-1(3)$ & $-19(3)$ \\
$\mathrm{C}(13)$ & $130(8)$ & $45(4)$ & $21(4)$ & $1(3)$ & $5(4)$ & $-39(4)$ \\
$\mathrm{C}(14)$ & $134(8)$ & $34(4)$ & $40(4)$ & $-12(3)$ & $40(5)$ & $-27(5)$ \\
$\mathrm{C}(15)$ & $92(6)$ & $36(4)$ & $58(5)$ & $-4(4)$ & $35(4)$ & $-9(3)$ \\
$\mathrm{C}(16)$ & $66(4)$ & $33(3)$ & $38(4)$ & $-6(3)$ & $18(3)$ & $-17(3)$ \\
$\mathrm{C}(17)$ & $161(8)$ & $54(5)$ & $28(4)$ & $16(3)$ & $-16(4)$ & $-32(5)$ \\
$\mathrm{C}(18)$ & $51(4)$ & $37(3)$ & $34(3)$ & $4(3)$ & $0(3)$ & $-15(3)$ \\
$\mathrm{C}(19)$ & $79(5)$ & $62(5)$ & $61(5)$ & $-23(4)$ & $-8(4)$ & $7(4)$ \\
$\mathrm{C}(20)$ & $70(5)$ & $61(4)$ & $79(5)$ & $36(4)$ & $-20(4)$ & $-39(4)$ \\
$\mathrm{C}(21)$ & $46(4)$ & $57(4)$ & $44(4)$ & $-5(3)$ & $1(3)$ & $-14(3)$ \\
$\mathrm{C}(22)$ & $53(5)$ & $48(4)$ & $176(10)$ & $49(6)$ & $0(6)$ & $-9(3)$ \\
& & & & & &
\end{tabular}




\begin{tabular}{|c|c|c|c|c|c|c|}
\hline$C(23)$ & $62(6)$ & $86(7)$ & $380(20)$ & $108(10)$ & $-40(9)$ & $-2(5)$ \\
\hline$C(24)$ & $93(7)$ & $78(6)$ & 142(9) & $-19(6)$ & $67(6)$ & $-10(5)$ \\
\hline$C(25)$ & $35(4)$ & $70(5)$ & $133(8)$ & $47(5)$ & $-18(4)$ & $-4(3)$ \\
\hline $\operatorname{Si}(2)$ & $43(1)$ & $26(1)$ & $35(1)$ & $5(1)$ & $2(1)$ & $3(1)$ \\
\hline $\mathrm{O}(2)$ & $44(2)$ & $26(2)$ & $25(2)$ & $-3(2)$ & $-4(2)$ & $3(2)$ \\
\hline$C(31)$ & $37(3)$ & $23(3)$ & $25(3)$ & $1(2)$ & $0(2)$ & $0(2)$ \\
\hline $\mathrm{C}(32)$ & $43(3)$ & $31(3)$ & $18(3)$ & $2(2)$ & $4(2)$ & $6(2)$ \\
\hline$C(33)$ & $64(4)$ & $32(3)$ & $22(3)$ & $4(3)$ & $6(3)$ & $14(3)$ \\
\hline$C(34)$ & $34(3)$ & $25(3)$ & $28(3)$ & $5(2)$ & $2(2)$ & $5(2)$ \\
\hline$C(35)$ & $45(4)$ & $24(3)$ & $34(3)$ & $2(2)$ & $-1(3)$ & $1(2)$ \\
\hline$C(36)$ & $43(4)$ & $43(4)$ & $39(3)$ & $5(3)$ & $-11(3)$ & $3(3)$ \\
\hline $\mathrm{C}(37)$ & $33(3)$ & $39(3)$ & $41(4)$ & $3(3)$ & $-4(3)$ & $6(3)$ \\
\hline$C(38)$ & $48(4)$ & $25(3)$ & $39(3)$ & $0(3)$ & $-1(3)$ & $10(3)$ \\
\hline C(39) & $42(3)$ & $35(3)$ & $24(3)$ & $3(3)$ & $-2(2)$ & $-3(3)$ \\
\hline $\mathrm{C}(40)$ & 41(3) & $40(3)$ & 27(3) & 1(3) & $4(3)$ & 2(3) \\
\hline $\mathrm{C}(41)$ & $54(4)$ & $34(3)$ & $22(3)$ & 1(3) & $-6(3)$ & $13(3)$ \\
\hline$C(42)$ & $73(5)$ & $39(3)$ & 23(3) & $-3(3)$ & $1(3)$ & $17(3)$ \\
\hline $\mathrm{C}(43)$ & $114(7)$ & $38(4)$ & $25(3)$ & 1(3) & $6(4)$ & $32(4)$ \\
\hline$C(44)$ & $132(8)$ & $29(4)$ & $44(5)$ & $0(3)$ & $-35(5)$ & $20(4)$ \\
\hline $\mathrm{C}(45)$ & $82(5)$ & $37(4)$ & $57(5)$ & $-8(4)$ & $-34(4)$ & $15(3)$ \\
\hline $\mathrm{C}(46)$ & $59(4)$ & $37(3)$ & 41(4) & $-3(3)$ & $-18(3)$ & $11(3)$ \\
\hline$C(47)$ & $148(8)$ & $46(4)$ & $27(4)$ & 11(3) & $19(4)$ & $30(4)$ \\
\hline $\mathrm{C}(48)$ & $49(4)$ & $29(3)$ & $33(3)$ & $0(2)$ & $2(3)$ & $6(3)$ \\
\hline $\mathrm{C}(49)$ & $71(5)$ & $65(5)$ & $64(5)$ & $31(4)$ & $19(4)$ & $33(4)$ \\
\hline $\mathrm{C}(50)$ & $76(5)$ & $48(4)$ & $37(4)$ & $-11(3)$ & 11(3) & $-12(3)$ \\
\hline $\mathrm{C}(51)$ & $49(4)$ & $49(4)$ & $41(4)$ & $-2(3)$ & $4(3)$ & $11(3)$ \\
\hline $\mathrm{C}(52)$ & $52(4)$ & $39(4)$ & $147(8)$ & $38(5)$ & $-2(5)$ & $8(3)$ \\
\hline $\mathrm{C}(53)$ & $66(6)$ & $86(7)$ & $340(20)$ & $-119(10)$ & $-70(9)$ & $19(5)$ \\
\hline C(54) & $49(5)$ & $121(8)$ & $410(20)$ & $195(12)$ & $28(8)$ & $4(5)$ \\
\hline$C(55)$ & $47(4)$ & $65(5)$ & $98(6)$ & $41(5)$ & $18(4)$ & $6(3)$ \\
\hline
\end{tabular}


Table 5. Hydrogen coordinates ( $\left.\mathrm{x} 10^{4}\right)$ and isotropic displacement parameters $\left(\AA^{2} \times 10^{3}\right)$ for $\mathbf{6 a}$.

\begin{tabular}{|c|c|c|c|c|}
\hline & $\mathrm{x}$ & $\mathrm{y}$ & $\mathrm{z}$ & $\mathrm{U}(\mathrm{eq})$ \\
\hline $\mathrm{H}(5 \mathrm{~A})$ & 10946 & 1335 & 4274 & 38 \\
\hline $\mathrm{H}(6 \mathrm{~A})$ & 12173 & 1644 & 3331 & 46 \\
\hline $\mathrm{H}(7 \mathrm{~A})$ & 12344 & 2183 & 4298 & 45 \\
\hline $\mathrm{H}(8 \mathrm{~A})$ & 11254 & 2409 & 6205 & 45 \\
\hline $\mathrm{H}(9 \mathrm{~A})$ & 10014 & 2094 & 7192 & 36 \\
\hline $\mathrm{H}(10 \mathrm{~A})$ & 8778 & 1632 & 4207 & 52 \\
\hline $\mathrm{H}(10 \mathrm{~B})$ & 8543 & 1834 & 5980 & 52 \\
\hline $\mathrm{H}(10 \mathrm{C})$ & 8150 & 1473 & 5722 & 52 \\
\hline $\mathrm{H}(12 \mathrm{~A})$ & 10448 & 1798 & 10669 & 60 \\
\hline $\mathrm{H}(13 \mathrm{~A})$ & 10058 & 2236 & 12546 & 78 \\
\hline $\mathrm{H}(14 \mathrm{~A})$ & 8559 & 2432 & 12591 & 83 \\
\hline $\mathrm{H}(15 \mathrm{~A})$ & 7469 & 2206 & 10720 & 74 \\
\hline $\mathrm{H}(16 \mathrm{~A})$ & 7843 & 1762 & 8875 & 55 \\
\hline $\mathrm{H}(17 \mathrm{~A})$ & 9316 & 1279 & 11803 & 122 \\
\hline $\mathrm{H}(17 \mathrm{~B})$ & 9896 & 951 & 11556 & 122 \\
\hline $\mathrm{H}(17 \mathrm{C})$ & 8811 & 944 & 11322 & 122 \\
\hline $\mathrm{H}(19 \mathrm{~A})$ & 8188 & 256 & 4992 & 101 \\
\hline $\mathrm{H}(19 \mathrm{~B})$ & 9279 & 270 & 5061 & 101 \\
\hline $\mathrm{H}(19 \mathrm{C})$ & 8717 & 563 & 4175 & 101 \\
\hline $\mathrm{H}(20 \mathrm{~A})$ & 8061 & 175 & 8200 & 105 \\
\hline $\mathrm{H}(20 \mathrm{~B})$ & 8494 & 430 & 9580 & 105 \\
\hline $\mathrm{H}(20 \mathrm{C})$ & 9143 & 186 & 8495 & 105 \\
\hline $\mathrm{H}(21 \mathrm{~A})$ & 7261 & 635 & 6738 & 73 \\
\hline $\mathrm{H}(21 \mathrm{~B})$ & 7822 & 940 & 5978 & 73 \\
\hline $\mathrm{H}(21 \mathrm{C})$ & 7727 & 888 & 8089 & 73 \\
\hline $\mathrm{H}(23 \mathrm{~A})$ & 11502 & 270 & 8323 & 264 \\
\hline $\mathrm{H}(23 \mathrm{~B})$ & 10436 & 202 & 8041 & 264 \\
\hline $\mathrm{H}(23 \mathrm{C})$ & 10773 & 422 & 9682 & 264 \\
\hline $\mathrm{H}(24 \mathrm{~A})$ & 11641 & 437 & 5169 & 157 \\
\hline
\end{tabular}




\begin{tabular}{|c|c|c|c|c|}
\hline $\mathrm{H}(24 \mathrm{~B})$ & 11064 & 742 & 4466 & 157 \\
\hline $\mathrm{H}(24 \mathrm{C})$ & 10571 & 399 & 4783 & 157 \\
\hline $\mathrm{H}(25 \mathrm{~A})$ & 12142 & 821 & 7848 & 119 \\
\hline $\mathrm{H}(25 \mathrm{~B})$ & 11390 & 998 & 9055 & 119 \\
\hline $\mathrm{H}(25 \mathrm{C})$ & 11504 & 1097 & 6998 & 119 \\
\hline $\mathrm{H}(35 \mathrm{~A})$ & 3564 & 1335 & -741 & 41 \\
\hline $\mathrm{H}(36 \mathrm{~A})$ & 2341 & 1653 & -1718 & 50 \\
\hline $\mathrm{H}(37 \mathrm{~A})$ & 2164 & 2184 & -720 & 45 \\
\hline $\mathrm{H}(38 \mathrm{~A})$ & 3229 & 2409 & 1270 & 45 \\
\hline $\mathrm{H}(39 \mathrm{~A})$ & 4477 & 2097 & 2180 & 40 \\
\hline $\mathrm{H}(40 \mathrm{~A})$ & 5720 & 1644 & -782 & 54 \\
\hline $\mathrm{H}(40 \mathrm{~B})$ & 6356 & 1489 & 736 & 54 \\
\hline $\mathrm{H}(40 \mathrm{C})$ & 5938 & 1846 & 996 & 54 \\
\hline $\mathrm{H}(42 \mathrm{~A})$ & 4077 & 1806 & 5671 & 54 \\
\hline $\mathrm{H}(43 \mathrm{~A})$ & 4457 & 2238 & 7550 & 71 \\
\hline $\mathrm{H}(44 \mathrm{~A})$ & 5926 & 2440 & 7587 & 82 \\
\hline $\mathrm{H}(45 \mathrm{~A})$ & 7057 & 2211 & 5779 & 70 \\
\hline $\mathrm{H}(46 \mathrm{~A})$ & 6682 & 1772 & 3915 & 55 \\
\hline $\mathrm{H}(47 \mathrm{~A})$ & 5254 & 1285 & 6786 & 111 \\
\hline $\mathrm{H}(47 \mathrm{~B})$ & 5734 & 946 & 6284 & 111 \\
\hline $\mathrm{H}(47 \mathrm{C})$ & 4652 & 962 & 6568 & 111 \\
\hline $\mathrm{H}(49 \mathrm{~A})$ & 6467 & 169 & 2976 & 99 \\
\hline H(49B) & 5382 & 175 & 3230 & 99 \\
\hline $\mathrm{H}(49 \mathrm{C})$ & 6018 & 398 & 4466 & 99 \\
\hline $\mathrm{H}(50 \mathrm{~A})$ & 6333 & 288 & -180 & 81 \\
\hline $\mathrm{H}(50 \mathrm{~B})$ & 5830 & 610 & -877 & 81 \\
\hline $\mathrm{H}(50 \mathrm{C})$ & 5244 & 311 & -111 & 81 \\
\hline $\mathrm{H}(51 \mathrm{~A})$ & 7268 & 647 & 1772 & 70 \\
\hline $\mathrm{H}(51 \mathrm{~B})$ & 6790 & 889 & 3160 & 70 \\
\hline $\mathrm{H}(51 \mathrm{C})$ & 6712 & 956 & 1059 & 70 \\
\hline $\mathrm{H}(53 \mathrm{~A})$ & 2857 & 411 & 624 & 247 \\
\hline $\mathrm{H}(53 \mathrm{~B})$ & 3916 & 359 & 183 & 247 \\
\hline $\mathrm{H}(53 \mathrm{C})$ & 3426 & 694 & -335 & 247 \\
\hline $\mathrm{H}(54 \mathrm{~A})$ & 3074 & 283 & 3721 & 290 \\
\hline $\mathrm{H}(54 \mathrm{~B})$ & 3807 & 471 & 4924 & 290 \\
\hline
\end{tabular}


$\mathrm{H}(54 \mathrm{C})$

$\mathrm{H}(55 \mathrm{~A})$

$\mathrm{H}(55 \mathrm{~B})$

$\mathrm{H}(55 \mathrm{C})$
4141

2401

3022

3166
220

824

1095

1012
3421

2920

1974

4045
290

105

105

105 
Table 6. Torsion angles $\left[{ }^{\circ}\right]$ for $\mathbf{6 a}$.

\begin{tabular}{lc}
\hline $\mathrm{C}(3)-\mathrm{Si}(1)-\mathrm{O}(1)-\mathrm{C}(1)$ & $1.3(4)$ \\
$\mathrm{C}(18)-\mathrm{Si}(1)-\mathrm{O}(1)-\mathrm{C}(1)$ & $118.1(4)$ \\
$\mathrm{C}(22)-\mathrm{Si}(1)-\mathrm{O}(1)-\mathrm{C}(1)$ & $-116.6(5)$ \\
$\mathrm{Si}(1)-\mathrm{O}(1)-\mathrm{C}(1)-\mathrm{C}(10)$ & $-122.3(4)$ \\
$\mathrm{Si}(1)-\mathrm{O}(1)-\mathrm{C}(1)-\mathrm{C}(4)$ & $119.7(4)$ \\
$\mathrm{Si}(1)-\mathrm{O}(1)-\mathrm{C}(1)-\mathrm{C}(2)$ & $-2.3(5)$ \\
$\mathrm{O}(1)-\mathrm{C}(1)-\mathrm{C}(2)-\mathrm{C}(3)$ & $2.7(7)$ \\
$\mathrm{C}(10)-\mathrm{C}(1)-\mathrm{C}(2)-\mathrm{C}(3)$ & $119.5(5)$ \\
$\mathrm{C}(4)-\mathrm{C}(1)-\mathrm{C}(2)-\mathrm{C}(3)$ & $-116.0(5)$ \\
$\mathrm{O}(1)-\mathrm{C}(1)-\mathrm{C}(2)-\mathrm{C}(11)$ & $-174.5(4)$ \\
$\mathrm{C}(10)-\mathrm{C}(1)-\mathrm{C}(2)-\mathrm{C}(11)$ & $-57.7(6)$ \\
$\mathrm{C}(4)-\mathrm{C}(1)-\mathrm{C}(2)-\mathrm{C}(11)$ & $66.8(6)$ \\
$\mathrm{C}(11)-\mathrm{C}(2)-\mathrm{C}(3)-\mathrm{C}(17)$ & $-2.3(10)$ \\
$\mathrm{C}(1)-\mathrm{C}(2)-\mathrm{C}(3)-\mathrm{C}(17)$ & $-179.4(6)$ \\
$\mathrm{C}(11)-\mathrm{C}(2)-\mathrm{C}(3)-\mathrm{Si}(1)$ & $175.3(4)$ \\
$\mathrm{C}(1)-\mathrm{C}(2)-\mathrm{C}(3)-\mathrm{Si}(1)$ & $-1.9(6)$ \\
$\mathrm{O}(1)-\mathrm{Si}(1)-\mathrm{C}(3)-\mathrm{C}(2)$ & $0.4(4)$ \\
$\mathrm{C}(18)-\mathrm{Si}(1)-\mathrm{C}(3)-\mathrm{C}(2)$ & $-112.7(4)$ \\
$\mathrm{C}(22)-\mathrm{Si}(1)-\mathrm{C}(3)-\mathrm{C}(2)$ & $113.6(5)$ \\
$\mathrm{O}(1)-\mathrm{Si}(1)-\mathrm{C}(3)-\mathrm{C}(17)$ & $177.8(6)$ \\
$\mathrm{C}(18)-\mathrm{Si}(1)-\mathrm{C}(3)-\mathrm{C}(17)$ & $64.7(7)$ \\
$\mathrm{C}(22)-\mathrm{Si}(1)-\mathrm{C}(3)-\mathrm{C}(17)$ & $-68.9(7)$ \\
$\mathrm{O}(1)-\mathrm{C}(1)-\mathrm{C}(4)-\mathrm{C}(5)$ & $9.3(6)$ \\
$\mathrm{C}(10)-\mathrm{C}(1)-\mathrm{C}(4)-\mathrm{C}(5)$ & $-107.5(5)$ \\
$\mathrm{C}(2)-\mathrm{C}(1)-\mathrm{C}(4)-\mathrm{C}(5)$ & $126.5(5)$ \\
$\mathrm{O}(1)-\mathrm{C}(1)-\mathrm{C}(4)-\mathrm{C}(9)$ & $-175.9(5)$ \\
$\mathrm{C}(10)-\mathrm{C}(1)-\mathrm{C}(4)-\mathrm{C}(9)$ & $67.3(6)$ \\
$\mathrm{C}(2)-\mathrm{C}(1)-\mathrm{C}(4)-\mathrm{C}(9)$ & $-58.7(7)$ \\
$\mathrm{C}(9)-\mathrm{C}(4)-\mathrm{C}(5)-\mathrm{C}(6)$ & $1.8(8)$ \\
$\mathrm{C}(1)-\mathrm{C}(4)-\mathrm{C}(5)-\mathrm{C}(6)$ & $176.7(5)$ \\
$\mathrm{C}(4)-\mathrm{C}(5)-\mathrm{C}(6)-\mathrm{C}(7)$ & $-0.9(9)$ \\
$\mathrm{C}(5)-\mathrm{C}(6)-\mathrm{C}(7)-\mathrm{C}(8)$ & $-0.4(9)$ \\
$\mathrm{C}(6)-\mathrm{C}(7)-\mathrm{C}(8)-\mathrm{C}(9)$ & $0.8(8)$ \\
&
\end{tabular}




$\begin{array}{lc}\mathrm{C}(5)-\mathrm{C}(4)-\mathrm{C}(9)-\mathrm{C}(8) & -1.4(8) \\ \mathrm{C}(1)-\mathrm{C}(4)-\mathrm{C}(9)-\mathrm{C}(8) & -176.3(5) \\ \mathrm{C}(7)-\mathrm{C}(8)-\mathrm{C}(9)-\mathrm{C}(4) & 0.1(8) \\ \mathrm{C}(3)-\mathrm{C}(2)-\mathrm{C}(11)-\mathrm{C}(16) & -98.5(7) \\ \mathrm{C}(1)-\mathrm{C}(2)-\mathrm{C}(11)-\mathrm{C}(16) & 78.5(7) \\ \mathrm{C}(3)-\mathrm{C}(2)-\mathrm{C}(11)-\mathrm{C}(12) & 75.1(7) \\ \mathrm{C}(1)-\mathrm{C}(2)-\mathrm{C}(11)-\mathrm{C}(12) & -107.8(6) \\ \mathrm{C}(16)-\mathrm{C}(11)-\mathrm{C}(12)-\mathrm{C}(13) & -1.3(8) \\ \mathrm{C}(2)-\mathrm{C}(11)-\mathrm{C}(12)-\mathrm{C}(13) & -175.0(5) \\ \mathrm{C}(11)-\mathrm{C}(12)-\mathrm{C}(13)-\mathrm{C}(14) & 0.7(9) \\ \mathrm{C}(12)-\mathrm{C}(13)-\mathrm{C}(14)-\mathrm{C}(15) & -0.7(10) \\ \mathrm{C}(13)-\mathrm{C}(14)-\mathrm{C}(15)-\mathrm{C}(16) & 1.3(10) \\ \mathrm{C}(12)-\mathrm{C}(11)-\mathrm{C}(16)-\mathrm{C}(15) & 1.9(8) \\ \mathrm{C}(2)-\mathrm{C}(11)-\mathrm{C}(16)-\mathrm{C}(15) & 175.6(5) \\ \mathrm{C}(14)-\mathrm{C}(15)-\mathrm{C}(16)-\mathrm{C}(11) & -1.9(9) \\ \mathrm{O}(1)-\mathrm{Si}(1)-\mathrm{C}(18)-\mathrm{C}(19) & 60.9(5) \\ \mathrm{C}(3)-\mathrm{Si}(1)-\mathrm{C}(18)-\mathrm{C}(19) & 164.4(4) \\ \mathrm{C}(22)-\mathrm{Si}(1)-\mathrm{C}(18)-\mathrm{C}(19) & -61.6(6) \\ \mathrm{O}(1)-\mathrm{Si}(1)-\mathrm{C}(18)-\mathrm{C}(20) & -174.1(4) \\ \mathrm{C}(3)-\mathrm{Si}(1)-\mathrm{C}(18)-\mathrm{C}(20) & -70.6(5) \\ \mathrm{C}(22)-\mathrm{Si}(1)-\mathrm{C}(18)-\mathrm{C}(20) & 63.4(6) \\ \mathrm{O}(1)-\mathrm{Si}(1)-\mathrm{C}(18)-\mathrm{C}(21) & -56.4(4) \\ \mathrm{C}(3)-\mathrm{Si}(1)-\mathrm{C}(18)-\mathrm{C}(21) & 47.1(5) \\ \mathrm{C}(22)-\mathrm{Si}(1)-\mathrm{C}(18)-\mathrm{C}(21) & -178.9(5) \\ \mathrm{O}(1)-\mathrm{Si}(1)-\mathrm{C}(22)-\mathrm{C}(23) & -171.1(9) \\ \mathrm{C}(3)-\mathrm{Si}(1)-\mathrm{C}(22)-\mathrm{C}(23) & 85.0(9) \\ \mathrm{C}(18)-\mathrm{Si}(1)-\mathrm{C}(22)-\mathrm{C}(23) & -48.6(11) \\ \mathrm{O}(1)-\mathrm{Si}(1)-\mathrm{C}(22)-\mathrm{C}(25) & 63.5(7) \\ \mathrm{C}(3)-\mathrm{Si}(1)-\mathrm{C}(22)-\mathrm{C}(25) & -40.4(7) \\ \mathrm{C}(18)-\mathrm{Si}(1)-\mathrm{C}(22)-\mathrm{C}(25) & -174.0(6) \\ \mathrm{O}(1)-\mathrm{Si}(1)-\mathrm{C}(22)-\mathrm{C}(24) & -50.8(5) \\ \mathrm{C}(3)-\mathrm{Si}(1)-\mathrm{C}(22)-\mathrm{C}(24) & -154.7(4) \\ \mathrm{C}(18)-\mathrm{Si}(1)-\mathrm{C}(22)-\mathrm{C}(24) & 71.8(5) \\ \mathrm{C}(33)-\mathrm{Si}(2)-\mathrm{O}(2)-\mathrm{C}(31) & -2.3(4) \\ \mathrm{C}(48)-\mathrm{Si}(2)-\mathrm{O}(2)-\mathrm{C}(31) & \\ & -118.3(4)\end{array}$




$\begin{array}{lc}\mathrm{C}(52)-\mathrm{Si}(2)-\mathrm{O}(2)-\mathrm{C}(31) & 114.5(4) \\ \mathrm{Si}(2)-\mathrm{O}(2)-\mathrm{C}(31)-\mathrm{C}(34) & -120.5(4) \\ \mathrm{Si}(2)-\mathrm{O}(2)-\mathrm{C}(31)-\mathrm{C}(40) & 122.8(4) \\ \mathrm{Si}(2)-\mathrm{O}(2)-\mathrm{C}(31)-\mathrm{C}(32) & 3.3(5) \\ \mathrm{O}(2)-\mathrm{C}(31)-\mathrm{C}(32)-\mathrm{C}(33) & -3.2(7) \\ \mathrm{C}(34)-\mathrm{C}(31)-\mathrm{C}(32)-\mathrm{C}(33) & 117.8(5) \\ \mathrm{C}(40)-\mathrm{C}(31)-\mathrm{C}(32)-\mathrm{C}(33) & -120.4(5) \\ \mathrm{O}(2)-\mathrm{C}(31)-\mathrm{C}(32)-\mathrm{C}(41) & 173.8(4) \\ \mathrm{C}(34)-\mathrm{C}(31)-\mathrm{C}(32)-\mathrm{C}(41) & -65.2(7) \\ \mathrm{C}(40)-\mathrm{C}(31)-\mathrm{C}(32)-\mathrm{C}(41) & 56.6(6) \\ \mathrm{C}(41)-\mathrm{C}(32)-\mathrm{C}(33)-\mathrm{C}(47) & 2.0(9) \\ \mathrm{C}(31)-\mathrm{C}(32)-\mathrm{C}(33)-\mathrm{C}(47) & 178.9(6) \\ \mathrm{C}(41)-\mathrm{C}(32)-\mathrm{C}(33)-\mathrm{Si}(2) & -175.3(4) \\ \mathrm{C}(31)-\mathrm{C}(32)-\mathrm{C}(33)-\mathrm{Si}(2) & 1.7(6) \\ \mathrm{O}(2)-\mathrm{Si}(2)-\mathrm{C}(33)-\mathrm{C}(32) & 0.3(4) \\ \mathrm{C}(48)-\mathrm{Si}(2)-\mathrm{C}(33)-\mathrm{C}(32) & 112.5(4) \\ \mathrm{C}(52)-\mathrm{Si}(2)-\mathrm{C}(33)-\mathrm{C}(32) & -113.5(4) \\ \mathrm{O}(2)-\mathrm{Si}(2)-\mathrm{C}(33)-\mathrm{C}(47) & -176.8(6) \\ \mathrm{C}(48)-\mathrm{Si}(2)-\mathrm{C}(33)-\mathrm{C}(47) & -64.7(7) \\ \mathrm{C}(52)-\mathrm{Si}(2)-\mathrm{C}(33)-\mathrm{C}(47) & 69.4(7) \\ \mathrm{O}(2)-\mathrm{C}(31)-\mathrm{C}(34)-\mathrm{C}(39) & 175.8(5) \\ \mathrm{C}(40)-\mathrm{C}(31)-\mathrm{C}(34)-\mathrm{C}(39) & -67.6(6) \\ \mathrm{C}(32)-\mathrm{C}(31)-\mathrm{C}(34)-\mathrm{C}(39) & 56.1(7) \\ \mathrm{O}(2)-\mathrm{C}(31)-\mathrm{C}(34)-\mathrm{C}(35) & -7.8(7) \\ \mathrm{C}(40)-\mathrm{C}(31)-\mathrm{C}(34)-\mathrm{C}(35) & 108.8(5) \\ \mathrm{C}(32)-\mathrm{C}(31)-\mathrm{C}(34)-\mathrm{C}(35) & -127.5(5) \\ \mathrm{C}(39)-\mathrm{C}(34)-\mathrm{C}(35)-\mathrm{C}(36) & -0.4(8) \\ \mathrm{C}(31)-\mathrm{C}(34)-\mathrm{C}(35)-\mathrm{C}(36) & -177.0(5) \\ \mathrm{C}(34)-\mathrm{C}(35)-\mathrm{C}(36)-\mathrm{C}(37) & -0.6(9) \\ \mathrm{C}(35)-\mathrm{C}(36)-\mathrm{C}(37)-\mathrm{C}(38) & 0.4(9) \\ \mathrm{C}(36)-\mathrm{C}(37)-\mathrm{C}(38)-\mathrm{C}(39) & 0.6(8) \\ \mathrm{C}(35)-\mathrm{C}(34)-\mathrm{C}(39)-\mathrm{C}(38) & 1.4(8) \\ \mathrm{C}(31)-\mathrm{C}(34)-\mathrm{C}(39)-\mathrm{C}(38) & -1.6(8) \\ \mathrm{C}(37)-\mathrm{C}(38)-\mathrm{C}(39)-\mathrm{C}(34) & \\ \mathrm{C}(33)-\mathrm{C}(32)-\mathrm{C}(41)-\mathrm{C}(42) & \\ & \end{array}$




$\begin{array}{lc}\mathrm{C}(31)-\mathrm{C}(32)-\mathrm{C}(41)-\mathrm{C}(42) & 106.8(6) \\ \mathrm{C}(33)-\mathrm{C}(32)-\mathrm{C}(41)-\mathrm{C}(46) & 97.3(7) \\ \mathrm{C}(31)-\mathrm{C}(32)-\mathrm{C}(41)-\mathrm{C}(46) & -79.5(7) \\ \mathrm{C}(46)-\mathrm{C}(41)-\mathrm{C}(42)-\mathrm{C}(43) & 1.5(8) \\ \mathrm{C}(32)-\mathrm{C}(41)-\mathrm{C}(42)-\mathrm{C}(43) & 175.3(5) \\ \mathrm{C}(41)-\mathrm{C}(42)-\mathrm{C}(43)-\mathrm{C}(44) & -0.2(9) \\ \mathrm{C}(42)-\mathrm{C}(43)-\mathrm{C}(44)-\mathrm{C}(45) & -0.6(10) \\ \mathrm{C}(43)-\mathrm{C}(44)-\mathrm{C}(45)-\mathrm{C}(46) & 0.2(10) \\ \mathrm{C}(44)-\mathrm{C}(45)-\mathrm{C}(46)-\mathrm{C}(41) & 1.1(9) \\ \mathrm{C}(42)-\mathrm{C}(41)-\mathrm{C}(46)-\mathrm{C}(45) & -1.9(8) \\ \mathrm{C}(32)-\mathrm{C}(41)-\mathrm{C}(46)-\mathrm{C}(45) & -175.8(5) \\ \mathrm{O}(2)-\mathrm{Si}(2)-\mathrm{C}(48)-\mathrm{C}(51) & 58.5(4) \\ \mathrm{C}(33)-\mathrm{Si}(2)-\mathrm{C}(48)-\mathrm{C}(51) & -44.1(5) \\ \mathrm{C}(52)-\mathrm{Si}(2)-\mathrm{C}(48)-\mathrm{C}(51) & -177.4(5) \\ \mathrm{O}(2)-\mathrm{Si}(2)-\mathrm{C}(48)-\mathrm{C}(49) & 179.6(4) \\ \mathrm{C}(33)-\mathrm{Si}(2)-\mathrm{C}(48)-\mathrm{C}(49) & 77.0(5) \\ \mathrm{C}(52)-\mathrm{Si}(2)-\mathrm{C}(48)-\mathrm{C}(49) & -56.3(6) \\ \mathrm{O}(2)-\mathrm{Si}(2)-\mathrm{C}(48)-\mathrm{C}(50) & -57.9(4) \\ \mathrm{C}(33)-\mathrm{Si}(2)-\mathrm{C}(48)-\mathrm{C}(50) & -160.5(4) \\ \mathrm{C}(52)-\mathrm{Si}(2)-\mathrm{C}(48)-\mathrm{C}(50) & 66.2(5) \\ \mathrm{O}(2)-\mathrm{Si}(2)-\mathrm{C}(52)-\mathrm{C}(54) & 177.7(8) \\ \mathrm{C}(33)-\mathrm{Si}(2)-\mathrm{C}(52)-\mathrm{C}(54) & -78.8(8) \\ \mathrm{C}(48)-\mathrm{Si}(2)-\mathrm{C}(52)-\mathrm{C}(54) & 54.3(9) \\ \mathrm{O}(2)-\mathrm{Si}(2)-\mathrm{C}(52)-\mathrm{C}(55) & -61.7(7) \\ \mathrm{C}(33)-\mathrm{Si}(2)-\mathrm{C}(52)-\mathrm{C}(55) & 41.8(7) \\ \mathrm{C}(48)-\mathrm{Si}(2)-\mathrm{C}(52)-\mathrm{C}(55) & 174.9(5) \\ \mathrm{O}(2)-\mathrm{Si}(2)-\mathrm{C}(52)-\mathrm{C}(53) & 56.9(6) \\ \mathrm{C}(33)-\mathrm{Si}(2)-\mathrm{C}(52)-\mathrm{C}(53) & 160.4(5) \\ \mathrm{C}(48)-\mathrm{Si}(2)-\mathrm{C}(52)-\mathrm{C}(53) & -66.5(6) \\ & \end{array}$


X-ray Data Collection, Structure Solution and Refinement for 14.

A colorless crystal of approximate dimensions $0.15 \times 0.21 \times 0.30 \mathrm{~mm}$ was mounted on a glass fiber and transferred to a Bruker CCD platform diffractometer. The SMART ${ }^{1}$ program package was used to determine the unit-cell parameters and for data collection $(25 \mathrm{sec} /$ frame scan time for a sphere of diffraction data). The raw frame data was processed using SAINT ${ }^{2}$ and $\mathrm{SADABS}^{3}$ to yield the reflection data file. Subsequent calculations were carried out using the SHELXTL ${ }^{4}$ program. There were no systematic absences nor any diffraction symmetry other than the Friedel condition. The centrosymmetric triclinic space group $\mathrm{P} \overline{1}$ was assigned and later determined to be correct.

The structure was solved by direct methods and refined on $\mathrm{F}^{2}$ by full-matrix least-squares techniques. The analytical scattering factors ${ }^{5}$ for neutral atoms were used throughout the analysis. Hydrogen atoms were either located from a difference-Fourier map and refined ( $\mathrm{x}, \mathrm{y}, \mathrm{z}$ and $\left.\mathrm{U}_{\text {iso }}\right)$ or were included using a riding model. Carbon atoms $\mathrm{C}(17)-\mathrm{C}(25)$ were disordered and included using multiple components with partial siteoccupancy-factors. At convergence, $\mathrm{wR} 2=0.1324$ and Goof $=1.039$ for 563 variables refined against 8952 data. As a comparison for refinement on F, R1 $=0.0487$ for those 6432 data with $\mathrm{I}>2.0 \sigma(\mathrm{I})$.

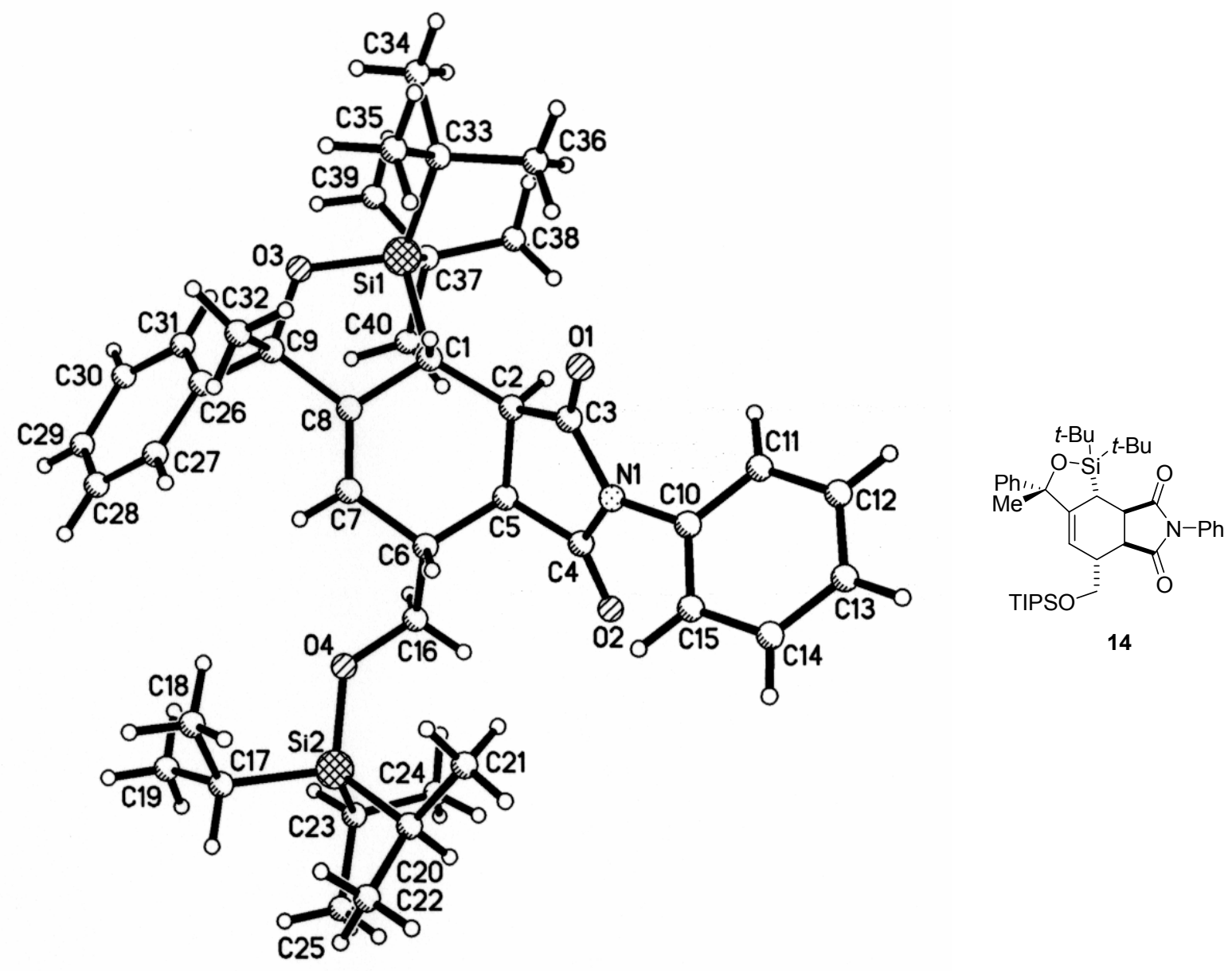




\section{References.}

6. SMART Software Users Guide, Version 5.1, Bruker Analytical X-Ray Systems, Inc.; Madison, WI 1999.

7. SAINT Software Users Guide, Version 6.0, Bruker Analytical X-Ray Systems, Inc.; Madison, WI 1999.

8. Sheldrick, G. M. SADABS, Version 2.05, Bruker Analytical X-Ray Systems, Inc.; Madison, WI 2001.

9. Sheldrick, G. M. SHELXTL Version 6.12, Bruker Analytical X-Ray Systems, Inc.; Madison, WI 2001.

10. International Tables for X-Ray Crystallography 1992, Vol. C., Dordrecht: Kluwer AcademicPublishers.

\section{Definitions:}

$\mathrm{wR} 2=\left[\Sigma\left[\mathrm{w}\left(\mathrm{F}_{\mathrm{o}}^{2}-\mathrm{F}_{\mathrm{c}}^{2}\right)^{2}\right] / \Sigma\left[\mathrm{w}\left(\mathrm{F}_{\mathrm{o}}^{2}\right)^{2}\right]\right]^{1 / 2}$

$\mathrm{R} 1=\Sigma|| \mathrm{F}_{\mathrm{o}}|-| \mathrm{F}_{\mathrm{c}}|| / \Sigma\left|\mathrm{F}_{\mathrm{o}}\right|$

Goof $=\mathrm{S}=\left[\Sigma\left[\mathrm{w}\left(\mathrm{F}_{\mathrm{o}}^{2}-\mathrm{F}_{\mathrm{c}}{ }^{2}\right)^{2}\right] /(\mathrm{n}-\mathrm{p})\right]^{1 / 2}$ where $\mathrm{n}$ is the number of reflections and $\mathrm{p}$ is the total number of parameters refined.

The thermal ellipsoid plot is shown at the $50 \%$ probability level. 
Table 1. Crystal data and structure refinement for $\mathbf{1 4}$.

Identification code

Empirical formula

Formula weight

Temperature

Wavelength

Crystal system

Space group

Unit cell dimensions

Volume

$\mathrm{Z}$

Density (calculated)

Absorption coefficient

$\mathrm{F}(000)$

Crystal size

Theta range for data collection

Index ranges

Reflections collected

Independent reflections

Completeness to theta $=28.31^{\circ}$

Absorption correction

Max. and min. transmission

Refinement method

Data / restraints / parameters

Goodness-of-fit on $\mathrm{F}^{2}$

Final $R$ indices [I $>2 \operatorname{sigma}(\mathrm{I})]$

$\mathrm{R}$ indices (all data)

Largest diff. peak and hole kaw53 (Tim Clark)

$\mathrm{C}_{40} \mathrm{H}_{59} \mathrm{~N} \mathrm{O}_{4} \mathrm{Si}_{2}$

674.06

163(2) K

$0.71073 \AA$

Triclinic

P $\overline{1}$

$a=9.034(2) \AA \quad \alpha=73.068(4)^{\circ}$.

$\mathrm{b}=14.237(4) \AA \quad \beta=89.726(5)^{\circ}$.

$\mathrm{c}=16.346(4) \AA \quad \gamma=73.451(4)^{\circ}$.

1920.7(9) $\AA^{3}$

2

$1.165 \mathrm{Mg} / \mathrm{m}^{3}$

$0.132 \mathrm{~mm}^{-1}$

732

$0.30 \times 0.21 \times 0.15 \mathrm{~mm}^{3}$

1.31 to $28.31^{\circ}$.

$-11 \leq h \leq 11,-18 \leq k \leq 18,-20 \leq l \leq 21$

20133

$8952[\mathrm{R}(\mathrm{int})=0.0332]$

$93.7 \%$

Semi-empirical from equivalents

0.9805 and 0.9615

Full-matrix least-squares on $\mathrm{F}^{2}$

8952 / 0 / 563

1.039

$\mathrm{R} 1=0.0487, \mathrm{wR} 2=0.1175$

$\mathrm{R} 1=0.0749, \mathrm{wR} 2=0.1324$

0.677 and -0.773 e. $\AA^{-3}$ 
Table 2. Atomic coordinates $\left(\mathrm{x} 10^{4}\right)$ and equivalent isotropic displacement parameters $\left(\AA^{2} \times 10^{3}\right)$ for 14. $U(e q)$ is defined as one third of the trace of the orthogonalized $U^{i j}$ tensor.

\begin{tabular}{lrrrr}
\hline & $\mathrm{x}$ & $\mathrm{y}$ & $\mathrm{z}$ & $\mathrm{U}(\mathrm{eq})$ \\
\hline $\mathrm{Si}(1)$ & $270(1)$ & $1832(1)$ & $3378(1)$ & $16(1)$ \\
$\mathrm{Si}(2)$ & $-3926(1)$ & $8085(1)$ & $1985(1)$ & $30(1)$ \\
$\mathrm{O}(1)$ & $-2413(2)$ & $2862(1)$ & $960(1)$ & $27(1)$ \\
$\mathrm{O}(2)$ & $-1372(2)$ & $5940(1)$ & $54(1)$ & $23(1)$ \\
$\mathrm{O}(3)$ & $-545(1)$ & $2052(1)$ & $4249(1)$ & $18(1)$ \\
$\mathrm{O}(4)$ & $-2865(2)$ & $6876(1)$ & $2340(1)$ & $25(1)$ \\
$\mathrm{N}(1)$ & $-2061(2)$ & $4446(1)$ & $315(1)$ & $19(1)$ \\
$\mathrm{C}(1)$ & $-1180(2)$ & $2985(1)$ & $2599(1)$ & $16(1)$ \\
$\mathrm{C}(2)$ & $-808(2)$ & $3544(1)$ & $1701(1)$ & $17(1)$ \\
$\mathrm{C}(3)$ & $-1861(2)$ & $3535(2)$ & $981(1)$ & $20(1)$ \\
$\mathrm{C}(4)$ & $-1418(2)$ & $5128(2)$ & $541(1)$ & $19(1)$ \\
$\mathrm{C}(5)$ & $-885(2)$ & $4687(1)$ & $1492(1)$ & $17(1)$ \\
$\mathrm{C}(6)$ & $-2062(2)$ & $5291(1)$ & $1982(1)$ & $17(1)$ \\
$\mathrm{C}(7)$ & $-2038(2)$ & $4674(1)$ & $2906(1)$ & $18(1)$ \\
$\mathrm{C}(8)$ & $-1713(2)$ & $3662(1)$ & $3173(1)$ & $16(1)$ \\
$\mathrm{C}(9)$ & $-1787(2)$ & $3004(1)$ & $4099(1)$ & $17(1)$ \\
$\mathrm{C}(10)$ & $-2696(2)$ & $4614(2)$ & $-537(1)$ & $20(1)$ \\
$\mathrm{C}(11)$ & $-2047(2)$ & $3905(2)$ & $-964(1)$ & $25(1)$ \\
$\mathrm{C}(12)$ & $-2619(3)$ & $4076(2)$ & $-1796(1)$ & $29(1)$ \\
$\mathrm{C}(13)$ & $-3826(3)$ & $4940(2)$ & $-2193(1)$ & $33(1)$ \\
$\mathrm{C}(14)$ & $-4463(3)$ & $5641(2)$ & $-1762(1)$ & $35(1)$ \\
$\mathrm{C}(15)$ & $-3906(2)$ & $5488(2)$ & $-926(1)$ & $28(1)$ \\
$\mathrm{C}(16)$ & $-1734(2)$ & $6304(2)$ & $1922(1)$ & $21(1)$ \\
$\mathrm{C}(17)$ & $-5087(6)$ & $8260(4)$ & $2903(3)$ & $25(2)$ \\
$\mathrm{C}(18)$ & $-5869(6)$ & $7367(4)$ & $3208(3)$ & $34(1)$ \\
$\mathrm{C}(19)$ & $-4226(5)$ & $8311(3)$ & $3661(3)$ & $29(1)$ \\
$\mathrm{C}(20)$ & $-5063(3)$ & $8479(2)$ & $932(2)$ & $36(1)$ \\
$\mathrm{C}(21)$ & $-5068(4)$ & $7596(3)$ & $579(2)$ & $44(1)$ \\
$\mathrm{C}(22)$ & $-6726(5)$ & $9138(3)$ & $916(3)$ & $52(1)$ \\
$\mathrm{C}(23)$ & $-2542(3)$ & $8946(2)$ & $1851(2)$ & $29(1)$ \\
& & & &
\end{tabular}




$\begin{array}{lrrrr}\mathrm{C}(24) & -1307(3) & 8734(2) & 1241(2) & 35(1) \\ \mathrm{C}(25) & -3489(4) & 10075(2) & 1584(2) & 33(1) \\ \mathrm{C}(17 \mathrm{~B}) & -5265(12) & 8181(8) & 2906(7) & 12(3) \\ \mathrm{C}(18 \mathrm{~B}) & -6214(12) & 7499(8) & 3093(7) & 28(3) \\ \mathrm{C}(19 \mathrm{~B}) & -4197(11) & 8025(8) & 3733(6) & 28(2) \\ \mathrm{C}(20 \mathrm{~B}) & -5503(10) & 7920(7) & 1132(6) & 14(2) \\ \mathrm{C}(21 \mathrm{~B}) & -4662(13) & 7718(8) & 301(7) & 23(2) \\ \mathrm{C}(22 \mathrm{~B}) & -6957(11) & 8791(8) & 905(6) & 17(2) \\ \mathrm{C}(23 \mathrm{~B}) & -3166(11) & 8946(7) & 1469(6) & 18(2) \\ \mathrm{C}(24 \mathrm{~B}) & -1432(14) & 8658(9) & 1782(8) & 35(3) \\ \mathrm{C}(25 \mathrm{~B}) & -4104(15) & 10081(9) & 1494(8) & 29(2) \\ \mathrm{C}(26) & -1583(2) & 3519(1) & 4775(1) & 19(1) \\ \mathrm{C}(27) & -2742(2) & 4378(2) & 4844(1) & 22(1) \\ \mathrm{C}(28) & -2542(3) & 4874(2) & 5433(1) & 27(1) \\ \mathrm{C}(29) & -1204(3) & 4506(2) & 5980(1) & 29(1) \\ \mathrm{C}(30) & -69(3) & 3637(2) & 5941(1) & 29(1) \\ \mathrm{C}(31) & -256(2) & 3148(2) & 5340(1) & 23(1) \\ \mathrm{C}(32) & -3320(2) & 2735(2) & 4177(1) & 22(1) \\ \mathrm{C}(33) & 77(2) & 590(2) & 3249(1) & 22(1) \\ \mathrm{C}(34) & 1227(3) & -368(2) & 3864(2) & 31(1) \\ \mathrm{C}(35) & -1565(3) & 520(2) & 3422(2) & 30(1) \\ \mathrm{C}(36) & 344(3) & 582(2) & 2317(1) & 29(1) \\ \mathrm{C}(37) & 2327(2) & 1913(2) & 3452(1) & 22(1) \\ \mathrm{C}(38) & 3299(3) & 1628(2) & 2740(2) & 32(1) \\ \mathrm{C}(39) & 3177(3) & 1207(2) & 4326(2) & 31(1) \\ \mathrm{C}(40) & 2219(2) & 3024(2) & 3406(2) & 27(1)\end{array}$


Table 3. Bond lengths $[\AA]$ and angles $\left[^{\circ}\right]$ for 14 .

\begin{tabular}{ll}
\hline $\mathrm{Si}(1)-\mathrm{O}(3)$ & $1.6680(14)$ \\
$\mathrm{Si}(1)-\mathrm{C}(33)$ & $1.896(2)$ \\
$\mathrm{Si}(1)-\mathrm{C}(1)$ & $1.8994(19)$ \\
$\mathrm{Si}(1)-\mathrm{C}(37)$ & $1.901(2)$ \\
$\mathrm{Si}(2)-\mathrm{C}(23 \mathrm{~B})$ & $1.598(9)$ \\
$\mathrm{Si}(2)-\mathrm{O}(4)$ & $1.6438(15)$ \\
$\mathrm{Si}(2)-\mathrm{C}(20)$ & $1.860(3)$ \\
$\mathrm{Si}(2)-\mathrm{C}(17)$ & $1.862(5)$ \\
$\mathrm{Si}(2)-\mathrm{C}(17 \mathrm{~B})$ & $1.938(10)$ \\
$\mathrm{Si}(2)-\mathrm{C}(23)$ & $1.956(3)$ \\
$\mathrm{Si}(2)-\mathrm{C}(20 \mathrm{~B})$ & $2.105(8)$ \\
$\mathrm{O}(1)-\mathrm{C}(3)$ & $1.209(2)$ \\
$\mathrm{O}(2)-\mathrm{C}(4)$ & $1.210(2)$ \\
$\mathrm{O}(3)-\mathrm{C}(9)$ & $1.449(2)$ \\
$\mathrm{O}(4)-\mathrm{C}(16)$ & $1.420(2)$ \\
$\mathrm{N}(1)-\mathrm{C}(3)$ & $1.395(2)$ \\
$\mathrm{N}(1)-\mathrm{C}(4)$ & $1.396(2)$ \\
$\mathrm{N}(1)-\mathrm{C}(10)$ & $1.436(2)$ \\
$\mathrm{C}(1)-\mathrm{C}(8)$ & $1.517(2)$ \\
$\mathrm{C}(1)-\mathrm{C}(2)$ & $1.539(3)$ \\
$\mathrm{C}(2)-\mathrm{C}(3)$ & $1.521(3)$ \\
$\mathrm{C}(2)-\mathrm{C}(5)$ & $1.543(3)$ \\
$\mathrm{C}(4)-\mathrm{C}(5)$ & $1.519(3)$ \\
$\mathrm{C}(5)-\mathrm{C}(6)$ & $1.545(2)$ \\
$\mathrm{C}(6)-\mathrm{C}(7)$ & $1.506(3)$ \\
$\mathrm{C}(6)-\mathrm{C}(16)$ & $1.529(3)$ \\
$\mathrm{C}(7)-\mathrm{C}(8)$ & $1.321(3)$ \\
$\mathrm{C}(8)-\mathrm{C}(9)$ & $1.542(3)$ \\
$\mathrm{C}(9)-\mathrm{C}(26)$ & $1.530(3)$ \\
$\mathrm{C}(9)-\mathrm{C}(32)$ & $1.534(3)$ \\
$\mathrm{C}(10)-\mathrm{C}(11)$ & $1.384(3)$ \\
$\mathrm{C}(10)-\mathrm{C}(15)$ & $1.385(3)$ \\
$\mathrm{C}(11)-\mathrm{C}(12)$ & \\
&
\end{tabular}




$\begin{array}{lc}\mathrm{C}(12)-\mathrm{C}(13) & 1.379(3) \\ \mathrm{C}(13)-\mathrm{C}(14) & 1.379(4) \\ \mathrm{C}(14)-\mathrm{C}(15) & 1.392(3) \\ \mathrm{C}(17)-\mathrm{C}(19) & 1.498(7) \\ \mathrm{C}(17)-\mathrm{C}(18) & 1.582(7) \\ \mathrm{C}(20)-\mathrm{C}(22) & 1.523(5) \\ \mathrm{C}(20)-\mathrm{C}(21) & 1.529(4) \\ \mathrm{C}(23)-\mathrm{C}(24) & 1.518(4) \\ \mathrm{C}(23)-\mathrm{C}(25) & 1.522(4) \\ \mathrm{C}(17 \mathrm{~B})-\mathrm{C}(18 \mathrm{~B}) & 1.439(15) \\ \mathrm{C}(17 \mathrm{~B})-\mathrm{C}(19 \mathrm{~B}) & 1.589(14) \\ \mathrm{C}(20 \mathrm{~B})-\mathrm{C}(22 \mathrm{~B}) & 1.487(12) \\ \mathrm{C}(20 \mathrm{~B})-\mathrm{C}(21 \mathrm{~B}) & 1.613(14) \\ \mathrm{C}(23 \mathrm{~B})-\mathrm{C}(24 \mathrm{~B}) & 1.550(15) \\ \mathrm{C}(23 \mathrm{~B})-\mathrm{C}(25 \mathrm{~B}) & 1.609(14) \\ \mathrm{C}(26)-\mathrm{C}(31) & 1.391(3) \\ \mathrm{C}(26)-\mathrm{C}(27) & 1.398(3) \\ \mathrm{C}(27)-\mathrm{C}(28) & 1.389(3) \\ \mathrm{C}(28)-\mathrm{C}(29) & 1.382(3) \\ \mathrm{C}(29)-\mathrm{C}(30) & 1.382(3) \\ \mathrm{C}(30)-\mathrm{C}(31) & 1.394(3) \\ \mathrm{C}(33)-\mathrm{C}(34) & 1.534(3) \\ \mathrm{C}(33)-\mathrm{C}(35) & 1.535(3) \\ \mathrm{C}(33)-\mathrm{C}(36) & 1.545(3) \\ \mathrm{C}(37)-\mathrm{C}(38) & 1.533(3) \\ \mathrm{C}(37)-\mathrm{C}(40) & 1.536(3) \\ \mathrm{C}(37)-\mathrm{C}(39) & 1.542(3) \\ \mathrm{O}(3)-\mathrm{Si}(1)-\mathrm{C}(33) & 110.82(8) \\ \mathrm{O}(3)-\mathrm{Si}(1)-\mathrm{C}(1) & 94.26(7) \\ \mathrm{C}(33)-\mathrm{Si}(1)-\mathrm{C}(1) & 110.64(9) \\ \mathrm{O}(3)-\mathrm{Si}(1)-\mathrm{C}(37) & 107.41(8) \\ \mathrm{C}(33)-\mathrm{Si}(1)-\mathrm{C}(37) & 115.75(9) \\ \mathrm{C}(1)-\mathrm{Si}(1)-\mathrm{C}(37) & 115.75(9) \\ \mathrm{C}(23 \mathrm{~B})-\mathrm{Si}(2)-\mathrm{O}(4) & 120.0(3) \\ & \\ & \end{array}$




$\begin{array}{lc}\mathrm{C}(23 \mathrm{~B})-\mathrm{Si}(2)-\mathrm{C}(20) & 81.7(4) \\ \mathrm{O}(4)-\mathrm{Si}(2)-\mathrm{C}(20) & 116.10(11) \\ \mathrm{C}(23 \mathrm{~B})-\mathrm{Si}(2)-\mathrm{C}(17) & 120.6(4) \\ \mathrm{O}(4)-\mathrm{Si}(2)-\mathrm{C}(17) & 102.97(18) \\ \mathrm{C}(20)-\mathrm{Si}(2)-\mathrm{C}(17) & 115.37(19) \\ \mathrm{C}(23 \mathrm{~B})-\mathrm{Si}(2)-\mathrm{C}(17 \mathrm{~B}) & 125.9(5) \\ \mathrm{O}(4)-\mathrm{Si}(2)-\mathrm{C}(17 \mathrm{~B}) & 101.2(3) \\ \mathrm{C}(20)-\mathrm{Si}(2)-\mathrm{C}(17 \mathrm{~B}) & 111.6(3) \\ \mathrm{C}(17)-\mathrm{Si}(2)-\mathrm{C}(17 \mathrm{~B}) & 6.2(4) \\ \mathrm{C}(23 \mathrm{~B})-\mathrm{Si}(2)-\mathrm{C}(23) & 25.0(3) \\ \mathrm{O}(4)-\mathrm{Si}(2)-\mathrm{C}(23) & 108.07(10) \\ \mathrm{C}(20)-\mathrm{Si}(2)-\mathrm{C}(23) & 106.60(13) \\ \mathrm{C}(17)-\mathrm{Si}(2)-\mathrm{C}(23) & 107.30(18) \\ \mathrm{C}(17 \mathrm{~B})-\mathrm{Si}(2)-\mathrm{C}(23) & 113.5(3) \\ \mathrm{C}(23 \mathrm{~B})-\mathrm{Si}(2)-\mathrm{C}(20 \mathrm{~B}) & 108.6(4) \\ \mathrm{O}(4)-\mathrm{Si}(2)-\mathrm{C}(20 \mathrm{~B}) & 98.1(2) \\ \mathrm{C}(20)-\mathrm{Si}(2)-\mathrm{C}(20 \mathrm{~B}) & 27.2(2) \\ \mathrm{C}(17)-\mathrm{Si}(2)-\mathrm{C}(20 \mathrm{~B}) & 103.2(3) \\ \mathrm{C}(17 \mathrm{~B})-\mathrm{Si}(2)-\mathrm{C}(20 \mathrm{~B}) & 97.6(4) \\ \mathrm{C}(23)-\mathrm{Si}(2)-\mathrm{C}(20 \mathrm{~B}) & 133.5(3) \\ \mathrm{C}(9)-\mathrm{O}(3)-\mathrm{Si}(1) & 115.75(11) \\ \mathrm{C}(16)-\mathrm{O}(4)-\mathrm{Si}(2) & 128.92(13) \\ \mathrm{C}(3)-\mathrm{N}(1)-\mathrm{C}(4) & 112.51(15) \\ \mathrm{C}(3)-\mathrm{N}(1)-\mathrm{C}(10) & 123.59(16) \\ \mathrm{C}(4)-\mathrm{N}(1)-\mathrm{C}(10) & 123.53(16) \\ \mathrm{C}(8)-\mathrm{C}(1)-\mathrm{C}(2) & 114.82(15) \\ \mathrm{C}(8)-\mathrm{C}(1)-\mathrm{Si}(1) & 100.41(12) \\ \mathrm{C}(2)-\mathrm{C}(1)-\mathrm{Si}(1) & 123.33(13) \\ \mathrm{C}(3)-\mathrm{C}(2)-\mathrm{C}(1) & 113.06(15) \\ \mathrm{C}(3)-\mathrm{C}(2)-\mathrm{C}(5) & 103.49(15) \\ \mathrm{C}(1)-\mathrm{C}(2)-\mathrm{C}(5) & 118.64(15) \\ \mathrm{O}(1)-\mathrm{C}(3)-\mathrm{N}(1) & 124.55(17) \\ \mathrm{O}(1)-\mathrm{C}(3)-\mathrm{C}(2) & 127.59(18) \\ \mathrm{N}(1)-\mathrm{C}(3)-\mathrm{C}(2) & 107.84(15) \\ \mathrm{O}(2)-\mathrm{C}(4)-\mathrm{N}(1) & 124.34(17) \\ & \end{array}$




$\begin{array}{ll}\mathrm{O}(2)-\mathrm{C}(4)-\mathrm{C}(5) & 127.73(17) \\ \mathrm{N}(1)-\mathrm{C}(4)-\mathrm{C}(5) & 107.86(15) \\ \mathrm{C}(4)-\mathrm{C}(5)-\mathrm{C}(2) & 103.65(14) \\ \mathrm{C}(4)-\mathrm{C}(5)-\mathrm{C}(6) & 108.33(15) \\ \mathrm{C}(2)-\mathrm{C}(5)-\mathrm{C}(6) & 114.42(15) \\ \mathrm{C}(7)-\mathrm{C}(6)-\mathrm{C}(16) & 110.67(15) \\ \mathrm{C}(7)-\mathrm{C}(6)-\mathrm{C}(5) & 111.92(15) \\ \mathrm{C}(16)-\mathrm{C}(6)-\mathrm{C}(5) & 109.68(15) \\ \mathrm{C}(8)-\mathrm{C}(7)-\mathrm{C}(6) & 123.76(17) \\ \mathrm{C}(7)-\mathrm{C}(8)-\mathrm{C}(1) & 123.70(17) \\ \mathrm{C}(7)-\mathrm{C}(8)-\mathrm{C}(9) & 125.68(16) \\ \mathrm{C}(1)-\mathrm{C}(8)-\mathrm{C}(9) & 110.62(15) \\ \mathrm{O}(3)-\mathrm{C}(9)-\mathrm{C}(26) & 109.23(14) \\ \mathrm{O}(3)-\mathrm{C}(9)-\mathrm{C}(32) & 107.53(15) \\ \mathrm{C}(26)-\mathrm{C}(9)-\mathrm{C}(32) & 110.06(15) \\ \mathrm{O}(3)-\mathrm{C}(9)-\mathrm{C}(8) & 106.57(14) \\ \mathrm{C}(26)-\mathrm{C}(9)-\mathrm{C}(8) & 113.28(15) \\ \mathrm{C}(32)-\mathrm{C}(9)-\mathrm{C}(8) & 109.96(15) \\ \mathrm{C}(11)-\mathrm{C}(10)-\mathrm{C}(15) & 121.28(19) \\ \mathrm{C}(11)-\mathrm{C}(10)-\mathrm{N}(1) & 118.77(18) \\ \mathrm{C}(15)-\mathrm{C}(10)-\mathrm{N}(1) & 119.92(18) \\ \mathrm{C}(10)-\mathrm{C}(11)-\mathrm{C}(12) & 119.3(2) \\ \mathrm{C}(13)-\mathrm{C}(12)-\mathrm{C}(11) & 120.3(2) \\ \mathrm{C}(14)-\mathrm{C}(13)-\mathrm{C}(12) & 119.9(2) \\ \mathrm{C}(13)-\mathrm{C}(14)-\mathrm{C}(15) & 120.9(2) \\ \mathrm{C}(10)-\mathrm{C}(15)-\mathrm{C}(14) & 118.4(2) \\ \mathrm{O}(4)-\mathrm{C}(16)-\mathrm{C}(6) & 109.34(15) \\ \mathrm{C}(19)-\mathrm{C}(17)-\mathrm{C}(18) & 109.0(4) \\ \mathrm{C}(19)-\mathrm{C}(17)-\mathrm{Si}(2) & 115.9(3) \\ \mathrm{C}(18)-\mathrm{C}(17)-\mathrm{Si}(2) & 109.4(3) \\ \mathrm{C}(22)-\mathrm{C}(20)-\mathrm{C}(21) & 109.2(3) \\ \mathrm{C}(22)-\mathrm{C}(20)-\mathrm{Si}(2) & 113.7(2) \\ \mathrm{C}(21)-\mathrm{C}(20)-\mathrm{Si}(2) & 114.9(2) \\ \mathrm{C}(24)-\mathrm{C}(23)-\mathrm{C}(25) & 111.5(2) \\ \mathrm{C}(24)-\mathrm{C}(23)-\mathrm{Si}(2) & 113.40(19)\end{array}$




$\begin{array}{ll}\mathrm{C}(25)-\mathrm{C}(23)-\mathrm{Si}(2) & 109.8(2) \\ \mathrm{C}(18 \mathrm{~B})-\mathrm{C}(17 \mathrm{~B})-\mathrm{C}(19 \mathrm{~B}) & 110.9(9) \\ \mathrm{C}(18 \mathrm{~B})-\mathrm{C}(17 \mathrm{~B})-\mathrm{Si}(2) & 116.6(7) \\ \mathrm{C}(19 \mathrm{~B})-\mathrm{C}(17 \mathrm{~B})-\mathrm{Si}(2) & 107.1(6) \\ \mathrm{C}(22 \mathrm{~B})-\mathrm{C}(20 \mathrm{~B})-\mathrm{C}(21 \mathrm{~B}) & 112.7(8) \\ \mathrm{C}(22 \mathrm{~B})-\mathrm{C}(20 \mathrm{~B})-\mathrm{Si}(2) & 112.9(6) \\ \mathrm{C}(21 \mathrm{~B})-\mathrm{C}(20 \mathrm{~B})-\mathrm{Si}(2) & 110.0(6) \\ \mathrm{C}(24 \mathrm{~B})-\mathrm{C}(23 \mathrm{~B})-\mathrm{Si}(2) & 111.0(7) \\ \mathrm{C}(24 \mathrm{~B})-\mathrm{C}(23 \mathrm{~B})-\mathrm{C}(25 \mathrm{~B}) & 111.5(9) \\ \mathrm{Si}(2)-\mathrm{C}(23 \mathrm{~B})-\mathrm{C}(25 \mathrm{~B}) & 113.0(7) \\ \mathrm{C}(31)-\mathrm{C}(26)-\mathrm{C}(27) & 117.96(18) \\ \mathrm{C}(31)-\mathrm{C}(26)-\mathrm{C}(9) & 121.50(17) \\ \mathrm{C}(27)-\mathrm{C}(26)-\mathrm{C}(9) & 120.54(17) \\ \mathrm{C}(28)-\mathrm{C}(27)-\mathrm{C}(26) & 120.94(19) \\ \mathrm{C}(29)-\mathrm{C}(28)-\mathrm{C}(27) & 120.25(19) \\ \mathrm{C}(28)-\mathrm{C}(29)-\mathrm{C}(30) & 119.64(19) \\ \mathrm{C}(29)-\mathrm{C}(30)-\mathrm{C}(31) & 120.2(2) \\ \mathrm{C}(26)-\mathrm{C}(31)-\mathrm{C}(30) & 120.99(19) \\ \mathrm{C}(34)-\mathrm{C}(33)-\mathrm{C}(35) & 107.81(18) \\ \mathrm{C}(34)-\mathrm{C}(33)-\mathrm{C}(36) & 108.89(17) \\ \mathrm{C}(35)-\mathrm{C}(33)-\mathrm{C}(36) & 108.04(17) \\ \mathrm{C}(34)-\mathrm{C}(33)-\mathrm{Si}(1) & 112.80(15) \\ \mathrm{C}(35)-\mathrm{C}(33)-\mathrm{Si}(1) & 109.89(14) \\ \mathrm{C}(36)-\mathrm{C}(33)-\mathrm{Si}(1) & 109.28(14) \\ \mathrm{C}(38)-\mathrm{C}(37)-\mathrm{C}(40) & 108.87(18) \\ \mathrm{C}(38)-\mathrm{C}(37)-\mathrm{C}(39) & 108.44(18) \\ \mathrm{C}(40)-\mathrm{C}(37)-\mathrm{C}(39) & 107.63(17) \\ \mathrm{C}(38)-\mathrm{C}(37)-\mathrm{Si}(1) & 114.24(14) \\ \mathrm{C}(40)-\mathrm{C}(37)-\mathrm{Si}(1) & 107.51(13) \\ \mathrm{C}(39)-\mathrm{C}(37)-\mathrm{Si}(1) & 109.95(14) \\ & \\ & \end{array}$


Table 4. Anisotropic displacement parameters $\left(\AA^{2} \times 10^{3}\right)$ for 14. The anisotropic displacement factor exponent takes the form: $-2 \pi^{2}\left[h^{2} a^{* 2} U^{11}+\ldots+2 h k a^{*} b^{*} U^{12}\right]$

\begin{tabular}{|c|c|c|c|c|c|c|}
\hline & $\mathrm{U}^{11}$ & $\mathrm{U}^{22}$ & $\mathrm{U}^{33}$ & $\mathrm{U}^{23}$ & $\mathrm{U}^{13}$ & $\mathrm{U}^{12}$ \\
\hline $\operatorname{Si}(1)$ & $17(1)$ & $16(1)$ & $15(1)$ & $-6(1)$ & $1(1)$ & $-3(1)$ \\
\hline $\operatorname{Si}(2)$ & $36(1)$ & $26(1)$ & $19(1)$ & $-5(1)$ & $7(1)$ & $4(1)$ \\
\hline $\mathrm{O}(1)$ & $36(1)$ & $28(1)$ & $19(1)$ & $-6(1)$ & $-1(1)$ & $-15(1)$ \\
\hline $\mathrm{O}(2)$ & $29(1)$ & 21(1) & $18(1)$ & $-4(1)$ & $7(1)$ & $-7(1)$ \\
\hline $\mathrm{O}(3)$ & $20(1)$ & $16(1)$ & $15(1)$ & $-4(1)$ & $1(1)$ & $-1(1)$ \\
\hline $\mathrm{O}(4)$ & $34(1)$ & $20(1)$ & $20(1)$ & $-9(1)$ & $8(1)$ & $-3(1)$ \\
\hline $\mathrm{N}(1)$ & $22(1)$ & $22(1)$ & $13(1)$ & $-5(1)$ & $3(1)$ & $-7(1)$ \\
\hline $\mathrm{C}(1)$ & $16(1)$ & $19(1)$ & $13(1)$ & $-5(1)$ & $2(1)$ & $-4(1)$ \\
\hline$C(2)$ & $17(1)$ & $19(1)$ & $13(1)$ & $-6(1)$ & $3(1)$ & $-3(1)$ \\
\hline$C(3)$ & $20(1)$ & $23(1)$ & $16(1)$ & $-7(1)$ & $6(1)$ & $-5(1)$ \\
\hline $\mathrm{C}(4)$ & $18(1)$ & $23(1)$ & $15(1)$ & $-8(1)$ & $6(1)$ & $-4(1)$ \\
\hline$C(5)$ & $17(1)$ & 19(1) & $16(1)$ & $-6(1)$ & $4(1)$ & $-7(1)$ \\
\hline $\mathrm{C}(6)$ & 19(1) & $18(1)$ & $15(1)$ & $-6(1)$ & $3(1)$ & $-4(1)$ \\
\hline$C(7)$ & $18(1)$ & 21(1) & $15(1)$ & $-7(1)$ & $4(1)$ & $-4(1)$ \\
\hline $\mathrm{C}(8)$ & $14(1)$ & $20(1)$ & $12(1)$ & $-6(1)$ & $1(1)$ & $-4(1)$ \\
\hline $\mathrm{C}(9)$ & $17(1)$ & $19(1)$ & $13(1)$ & $-4(1)$ & $2(1)$ & $-1(1)$ \\
\hline$C(10)$ & 21(1) & $30(1)$ & 11(1) & $-4(1)$ & $4(1)$ & $-12(1)$ \\
\hline $\mathrm{C}(11)$ & $25(1)$ & $30(1)$ & $20(1)$ & $-7(1)$ & $3(1)$ & $-11(1)$ \\
\hline$C(12)$ & $32(1)$ & $43(1)$ & $20(1)$ & $-13(1)$ & $6(1)$ & $-19(1)$ \\
\hline$C(13)$ & $33(1)$ & $55(2)$ & $15(1)$ & $-6(1)$ & $1(1)$ & $-23(1)$ \\
\hline$C(14)$ & $25(1)$ & $47(1)$ & 21(1) & $3(1)$ & $-3(1)$ & $-6(1)$ \\
\hline$C(15)$ & $24(1)$ & $36(1)$ & 21(1) & $-6(1)$ & $4(1)$ & $-6(1)$ \\
\hline$C(16)$ & $25(1)$ & $20(1)$ & $19(1)$ & $-7(1)$ & $6(1)$ & $-5(1)$ \\
\hline$C(26)$ & $21(1)$ & $20(1)$ & $15(1)$ & $-5(1)$ & $6(1)$ & $-7(1)$ \\
\hline$C(27)$ & $22(1)$ & $25(1)$ & $17(1)$ & $-5(1)$ & $5(1)$ & $-4(1)$ \\
\hline$C(28)$ & $32(1)$ & $25(1)$ & $23(1)$ & $-10(1)$ & $8(1)$ & $-3(1)$ \\
\hline$C(29)$ & $40(1)$ & $31(1)$ & $20(1)$ & $-13(1)$ & $5(1)$ & $-11(1)$ \\
\hline$C(30)$ & $30(1)$ & $35(1)$ & 21(1) & $-12(1)$ & $-3(1)$ & $-5(1)$ \\
\hline $\mathrm{C}(31)$ & $23(1)$ & $23(1)$ & 19(1) & $-7(1)$ & $0(1)$ & $-2(1)$ \\
\hline $\mathrm{C}(32)$ & $19(1)$ & $28(1)$ & $18(1)$ & $-6(1)$ & $4(1)$ & $-8(1)$ \\
\hline
\end{tabular}




$\begin{array}{lllllll}\mathrm{C}(33) & 28(1) & 19(1) & 21(1) & -8(1) & 3(1) & -6(1) \\ \mathrm{C}(34) & 41(1) & 20(1) & 29(1) & -5(1) & 0(1) & -4(1) \\ \mathrm{C}(35) & 36(1) & 28(1) & 34(1) & -13(1) & 7(1) & -17(1) \\ \mathrm{C}(36) & 36(1) & 31(1) & 24(1) & -15(1) & 4(1) & -10(1) \\ \mathrm{C}(37) & 19(1) & 24(1) & 22(1) & -8(1) & 0(1) & -4(1) \\ \mathrm{C}(38) & 22(1) & 43(1) & 34(1) & -17(1) & 8(1) & -7(1) \\ \mathrm{C}(39) & 23(1) & 32(1) & 33(1) & -10(1) & -5(1) & -2(1) \\ \mathrm{C}(40) & 19(1) & 30(1) & 34(1) & -11(1) & 1(1) & -10(1)\end{array}$


Table 5. Hydrogen coordinates ( $\left.\mathrm{x} 10^{4}\right)$ and isotropic displacement parameters $\left(\AA^{2} \times 10^{3}\right)$ for 14.

\begin{tabular}{|c|c|c|c|c|}
\hline & $\mathrm{x}$ & $\mathrm{y}$ & $\mathrm{z}$ & $\mathrm{U}(\mathrm{eq})$ \\
\hline $\mathrm{H}(6 \mathrm{~A})$ & -3123 & 5455 & 1699 & 21 \\
\hline $\mathrm{H}(17 \mathrm{~A})$ & -5939 & 8921 & 2687 & 30 \\
\hline $\mathrm{H}(18 \mathrm{~A})$ & -6489 & 7461 & 3687 & 51 \\
\hline $\mathrm{H}(18 \mathrm{~B})$ & -5062 & 6702 & 3394 & 51 \\
\hline $\mathrm{H}(18 \mathrm{C})$ & -6539 & 7383 & 2731 & 51 \\
\hline $\mathrm{H}(19 \mathrm{~A})$ & -4936 & 8403 & 4106 & 44 \\
\hline $\mathrm{H}(19 \mathrm{~B})$ & -3796 & 8892 & 3485 & 44 \\
\hline $\mathrm{H}(19 \mathrm{C})$ & -3381 & 7671 & 3890 & 44 \\
\hline $\mathrm{H}(20 \mathrm{~A})$ & -4547 & 8923 & 512 & 43 \\
\hline $\mathrm{H}(21 \mathrm{~A})$ & -5686 & 7872 & 24 & 66 \\
\hline $\mathrm{H}(21 \mathrm{~B})$ & -5517 & 7116 & 983 & 66 \\
\hline $\mathrm{H}(21 \mathrm{C})$ & -4002 & 7235 & 503 & 66 \\
\hline $\mathrm{H}(22 \mathrm{~A})$ & -7257 & 9319 & 345 & 79 \\
\hline $\mathrm{H}(22 \mathrm{~B})$ & -6722 & 9768 & 1046 & 79 \\
\hline $\mathrm{H}(22 \mathrm{C})$ & -7268 & 8753 & 1346 & 79 \\
\hline $\mathrm{H}(23 \mathrm{~A})$ & -1996 & 8787 & 2428 & 34 \\
\hline $\mathrm{H}(24 \mathrm{~A})$ & -649 & 9183 & 1205 & 53 \\
\hline $\mathrm{H}(24 \mathrm{~B})$ & -1804 & 8867 & 670 & 53 \\
\hline $\mathrm{H}(24 \mathrm{C})$ & -671 & 8014 & 1454 & 53 \\
\hline $\mathrm{H}(25 \mathrm{~A})$ & -2791 & 10502 & 1521 & 50 \\
\hline $\mathrm{H}(25 \mathrm{~B})$ & -4195 & 10202 & 2024 & 50 \\
\hline $\mathrm{H}(25 \mathrm{C})$ & -4091 & 10248 & 1036 & 50 \\
\hline $\mathrm{H}(17 \mathrm{~B})$ & -5978 & 8899 & 2743 & 15 \\
\hline $\mathrm{H}(18 \mathrm{D})$ & -6828 & 7607 & 3572 & 42 \\
\hline $\mathrm{H}(18 \mathrm{E})$ & -5550 & 6785 & 3251 & 42 \\
\hline $\mathrm{H}(18 \mathrm{~F})$ & -6910 & 7639 & 2586 & 42 \\
\hline $\mathrm{H}(19 \mathrm{D})$ & -4834 & 8058 & 4217 & 43 \\
\hline $\mathrm{H}(19 \mathrm{E})$ & -3690 & 8569 & 3618 & 43 \\
\hline $\mathrm{H}(19 \mathrm{~F})$ & -3408 & 7354 & 3875 & 43 \\
\hline
\end{tabular}




\begin{tabular}{|c|c|c|c|c|}
\hline $\mathrm{H}(20 \mathrm{~B})$ & -5798 & 7288 & 1435 & 17 \\
\hline $\mathrm{H}(21 \mathrm{D})$ & -5404 & 7643 & -93 & 34 \\
\hline $\mathrm{H}(21 \mathrm{E})$ & -3787 & 7088 & 480 & 34 \\
\hline $\mathrm{H}(21 \mathrm{~F})$ & -4284 & 8300 & 10 & 34 \\
\hline H(22D) & -7645 & 8677 & 506 & 26 \\
\hline $\mathrm{H}(22 \mathrm{E})$ & -6711 & 9433 & 632 & 26 \\
\hline $\mathrm{H}(22 \mathrm{~F})$ & -7475 & 8838 & 1426 & 26 \\
\hline $\mathrm{H}(23 \mathrm{~B})$ & -3208 & 8958 & 854 & 22 \\
\hline H(24D) & -901 & 7958 & 1774 & 53 \\
\hline $\mathrm{H}(24 \mathrm{E})$ & -1345 & 8697 & 2368 & 53 \\
\hline $\mathrm{H}(24 \mathrm{~F})$ & -953 & 9139 & 1401 & 53 \\
\hline $\mathrm{H}(25 \mathrm{D})$ & -3587 & 10575 & 1171 & 43 \\
\hline $\mathrm{H}(25 \mathrm{E})$ & -4133 & 10091 & 2091 & 43 \\
\hline $\mathrm{H}(25 \mathrm{~F})$ & -5165 & 10268 & 1236 & 43 \\
\hline $\mathrm{H}(1)$ & $-2040(20)$ & $2701(16)$ & 2511(13) & $20(5)$ \\
\hline $\mathrm{H}(2)$ & $200(20)$ & $3215(15)$ & 1577(13) & $17(5)$ \\
\hline $\mathrm{H}(5)$ & $110(20)$ & $4773(16)$ & $1580(13)$ & $18(5)$ \\
\hline $\mathrm{H}(7)$ & $-2310(20)$ & $5078(16)$ & $3281(14)$ & $23(6)$ \\
\hline $\mathrm{H}(11)$ & $-1190(30)$ & $3319(19)$ & $-676(15)$ & $33(6)$ \\
\hline $\mathrm{H}(12)$ & $-2200(30)$ & $3581(18)$ & $-2091(15)$ & $28(6)$ \\
\hline $\mathrm{H}(13)$ & $-4240(30)$ & $5052(19)$ & $-2772(17)$ & $39(7)$ \\
\hline $\mathrm{H}(14)$ & $-5240(30)$ & $6250(20)$ & $-1995(17)$ & $38(7)$ \\
\hline $\mathrm{H}(15)$ & $-4330(30)$ & 5996(19) & $-614(16)$ & $38(7)$ \\
\hline $\mathrm{H}(16 \mathrm{~A})$ & $-1700(20)$ & $6677(17)$ & $1339(15)$ & $22(5)$ \\
\hline $\mathrm{H}(16 \mathrm{~B})$ & $-700(30)$ & $6125(17)$ & $2204(15)$ & $28(6)$ \\
\hline $\mathrm{H}(27)$ & $-3700(30)$ & $4623(18)$ & $4476(15)$ & $31(6)$ \\
\hline $\mathrm{H}(28)$ & $-3370(30)$ & $5463(17)$ & $5470(14)$ & $25(6)$ \\
\hline $\mathrm{H}(29)$ & $-1060(30)$ & $4851(17)$ & $6376(15)$ & $27(6)$ \\
\hline $\mathrm{H}(30)$ & $880(30)$ & $3377(19)$ & $6329(17)$ & $42(7)$ \\
\hline $\mathrm{H}(31)$ & $520(20)$ & $2552(17)$ & $5322(14)$ & $21(5)$ \\
\hline $\mathrm{H}(32 \mathrm{~A})$ & $-3290(20)$ & $2249(17)$ & $4731(15)$ & $21(5)$ \\
\hline $\mathrm{H}(32 \mathrm{~B})$ & $-4180(30)$ & $3339(18)$ & $4119(14)$ & $26(6)$ \\
\hline $\mathrm{H}(32 \mathrm{C})$ & $-3470(30)$ & $2406(18)$ & $3738(16)$ & $32(6)$ \\
\hline $\mathrm{H}(34 \mathrm{~B})$ & $2360(30)$ & $-404(17)$ & $3760(15)$ & $28(6)$ \\
\hline $\mathrm{H}(34 \mathrm{C})$ & $1030(30)$ & $-980(20)$ & $3774(18)$ & $49(8)$ \\
\hline
\end{tabular}




\begin{tabular}{lrrrl}
$\mathrm{H}(34 \mathrm{~A})$ & $1120(30)$ & $-380(20)$ & $4463(18)$ & $40(7)$ \\
$\mathrm{H}(35 \mathrm{~B})$ & $-1630(30)$ & $-130(20)$ & $3350(16)$ & $38(7)$ \\
$\mathrm{H}(35 \mathrm{~A})$ & $-2350(30)$ & $1090(20)$ & $3007(17)$ & $36(7)$ \\
$\mathrm{H}(35 \mathrm{C})$ & $-1790(30)$ & $518(19)$ & $4040(17)$ & $40(7)$ \\
$\mathrm{H}(36 \mathrm{~A})$ & $210(30)$ & $-50(20)$ & $2237(16)$ & $36(7)$ \\
$\mathrm{H}(36 \mathrm{~B})$ & $1380(30)$ & $619(18)$ & $2158(16)$ & $35(6)$ \\
$\mathrm{H}(36 \mathrm{C})$ & $-420(30)$ & $1140(20)$ & $1918(17)$ & $36(7)$ \\
$\mathrm{H}(38 \mathrm{C})$ & $3480(30)$ & $900(20)$ & $2777(16)$ & $35(6)$ \\
$\mathrm{H}(38 \mathrm{~A})$ & $4310(30)$ & $1760(20)$ & $2774(17)$ & $46(7)$ \\
$\mathrm{H}(38 \mathrm{~B})$ & $2800(30)$ & $2080(20)$ & $2136(18)$ & $42(7)$ \\
$\mathrm{H}(39 \mathrm{~A})$ & $3430(30)$ & $460(20)$ & $4374(16)$ & $35(6)$ \\
$\mathrm{H}(39 B)$ & $2580(30)$ & $1333(18)$ & $4807(16)$ & $32(6)$ \\
$\mathrm{H}(39 \mathrm{C})$ & $4180(30)$ & $1331(19)$ & $4405(16)$ & $40(7)$ \\
$\mathrm{H}(40 \mathrm{~A})$ & $3260(30)$ & $3083(18)$ & $3480(16)$ & $35(6)$ \\
$\mathrm{H}(40 \mathrm{C})$ & $1580(30)$ & $3271(18)$ & $3843(15)$ & $30(6)$ \\
$\mathrm{H}(40 B)$ & $1720(30)$ & $3539(19)$ & $2828(17)$ & $36(7)$ \\
\hline
\end{tabular}


Table 6. Torsion angles $\left[{ }^{\circ}\right]$ for $\mathbf{1 4}$.

\begin{tabular}{lc}
\hline $\mathrm{C}(33)-\mathrm{Si}(1)-\mathrm{O}(3)-\mathrm{C}(9)$ & $-120.79(13)$ \\
$\mathrm{C}(1)-\mathrm{Si}(1)-\mathrm{O}(3)-\mathrm{C}(9)$ & $-6.81(13)$ \\
$\mathrm{C}(37)-\mathrm{Si}(1)-\mathrm{O}(3)-\mathrm{C}(9)$ & $111.88(13)$ \\
$\mathrm{C}(23 \mathrm{~B})-\mathrm{Si}(2)-\mathrm{O}(4)-\mathrm{C}(16)$ & $-41.9(4)$ \\
$\mathrm{C}(20)-\mathrm{Si}(2)-\mathrm{O}(4)-\mathrm{C}(16)$ & $53.7(2)$ \\
$\mathrm{C}(17)-\mathrm{Si}(2)-\mathrm{O}(4)-\mathrm{C}(16)$ & $-179.3(2)$ \\
$\mathrm{C}(17 \mathrm{~B})-\mathrm{Si}(2)-\mathrm{O}(4)-\mathrm{C}(16)$ & $174.6(3)$ \\
$\mathrm{C}(23)-\mathrm{Si}(2)-\mathrm{O}(4)-\mathrm{C}(16)$ & $-65.96(19)$ \\
$\mathrm{C}(20 \mathrm{~B})-\mathrm{Si}(2)-\mathrm{O}(4)-\mathrm{C}(16)$ & $75.1(3)$ \\
$\mathrm{O}(3)-\mathrm{Si}(1)-\mathrm{C}(1)-\mathrm{C}(8)$ & $24.43(12)$ \\
$\mathrm{C}(33)-\mathrm{Si}(1)-\mathrm{C}(1)-\mathrm{C}(8)$ & $138.57(11)$ \\
$\mathrm{C}(37)-\mathrm{Si}(1)-\mathrm{C}(1)-\mathrm{C}(8)$ & $-87.23(13)$ \\
$\mathrm{O}(3)-\mathrm{Si}(1)-\mathrm{C}(1)-\mathrm{C}(2)$ & $153.59(14)$ \\
$\mathrm{C}(33)-\mathrm{Si}(1)-\mathrm{C}(1)-\mathrm{C}(2)$ & $-92.27(16)$ \\
$\mathrm{C}(37)-\mathrm{Si}(1)-\mathrm{C}(1)-\mathrm{C}(2)$ & $41.93(17)$ \\
$\mathrm{C}(8)-\mathrm{C}(1)-\mathrm{C}(2)-\mathrm{C}(3)$ & $-116.98(17)$ \\
$\mathrm{Si}(1)-\mathrm{C}(1)-\mathrm{C}(2)-\mathrm{C}(3)$ & $120.18(16)$ \\
$\mathrm{C}(8)-\mathrm{C}(1)-\mathrm{C}(2)-\mathrm{C}(5)$ & $4.4(2)$ \\
$\mathrm{Si}(1)-\mathrm{C}(1)-\mathrm{C}(2)-\mathrm{C}(5)$ & $-118.40(16)$ \\
$\mathrm{C}(4)-\mathrm{N}(1)-\mathrm{C}(3)-\mathrm{O}(1)$ & $174.35(18)$ \\
$\mathrm{C}(10)-\mathrm{N}(1)-\mathrm{C}(3)-\mathrm{O}(1)$ & $-12.4(3)$ \\
$\mathrm{C}(4)-\mathrm{N}(1)-\mathrm{C}(3)-\mathrm{C}(2)$ & $-7.3(2)$ \\
$\mathrm{C}(10)-\mathrm{N}(1)-\mathrm{C}(3)-\mathrm{C}(2)$ & $165.96(16)$ \\
$\mathrm{C}(1)-\mathrm{C}(2)-\mathrm{C}(3)-\mathrm{O}(1)$ & $-34.1(3)$ \\
$\mathrm{C}(5)-\mathrm{C}(2)-\mathrm{C}(3)-\mathrm{O}(1)$ & $-163.74(19)$ \\
$\mathrm{C}(1)-\mathrm{C}(2)-\mathrm{C}(3)-\mathrm{N}(1)$ & $147.59(15)$ \\
$\mathrm{C}(5)-\mathrm{C}(2)-\mathrm{C}(3)-\mathrm{N}(1)$ & $17.96(18)$ \\
$\mathrm{C}(3)-\mathrm{N}(1)-\mathrm{C}(4)-\mathrm{O}(2)$ & $175.62(17)$ \\
$\mathrm{C}(10)-\mathrm{N}(1)-\mathrm{C}(4)-\mathrm{O}(2)$ & $2.4(3)$ \\
$\mathrm{C}(3)-\mathrm{N}(1)-\mathrm{C}(4)-\mathrm{C}(5)$ & $-7.0(2)$ \\
$\mathrm{C}(10)-\mathrm{N}(1)-\mathrm{C}(4)-\mathrm{C}(5)$ & $179.74(16)$ \\
$\mathrm{O}(2)-\mathrm{C}(4)-\mathrm{C}(5)-\mathrm{C}(2)$ & $-164.91(18)$ \\
$\mathrm{N}(1)-\mathrm{C}(4)-\mathrm{C}(5)-\mathrm{C}(2)$ & $17.84(18)$ \\
& \\
& \\
&
\end{tabular}




\begin{tabular}{|c|c|}
\hline $\mathrm{O}(2)-\mathrm{C}(4)-\mathrm{C}(5)-\mathrm{C}(6)$ & $73.2(2)$ \\
\hline $\mathrm{N}(1)-\mathrm{C}(4)-\mathrm{C}(5)-\mathrm{C}(6)$ & $-104.08(16)$ \\
\hline$C(3)-C(2)-C(5)-C(4)$ & $-21.12(17)$ \\
\hline$C(1)-C(2)-C(5)-C(4)$ & $-147.27(16)$ \\
\hline$C(3)-C(2)-C(5)-C(6)$ & $96.64(17)$ \\
\hline$C(1)-C(2)-C(5)-C(6)$ & $-29.5(2)$ \\
\hline$C(4)-C(5)-C(6)-C(7)$ & $156.33(15)$ \\
\hline$C(2)-C(5)-C(6)-C(7)$ & $41.3(2)$ \\
\hline$C(4)-C(5)-C(6)-C(16)$ & $-80.43(18)$ \\
\hline$C(2)-C(5)-C(6)-C(16)$ & $164.51(16)$ \\
\hline$C(16)-C(6)-C(7)-C(8)$ & $-154.07(18)$ \\
\hline$C(5)-C(6)-C(7)-C(8)$ & $-31.4(2)$ \\
\hline$C(6)-C(7)-C(8)-C(1)$ & $5.9(3)$ \\
\hline$C(6)-C(7)-C(8)-C(9)$ & $-175.37(16)$ \\
\hline $\mathrm{C}(2)-\mathrm{C}(1)-\mathrm{C}(8)-\mathrm{C}(7)$ & $8.6(3)$ \\
\hline $\mathrm{Si}(1)-\mathrm{C}(1)-\mathrm{C}(8)-\mathrm{C}(7)$ & $143.03(16)$ \\
\hline $\mathrm{C}(2)-\mathrm{C}(1)-\mathrm{C}(8)-\mathrm{C}(9)$ & $-170.36(15)$ \\
\hline $\mathrm{Si}(1)-\mathrm{C}(1)-\mathrm{C}(8)-\mathrm{C}(9)$ & $-35.90(16)$ \\
\hline $\mathrm{Si}(1)-\mathrm{O}(3)-\mathrm{C}(9)-\mathrm{C}(26)$ & $-136.17(12)$ \\
\hline $\mathrm{Si}(1)-\mathrm{O}(3)-\mathrm{C}(9)-\mathrm{C}(32)$ & $104.41(14)$ \\
\hline $\mathrm{Si}(1)-\mathrm{O}(3)-\mathrm{C}(9)-\mathrm{C}(8)$ & $-13.45(17)$ \\
\hline $\mathrm{C}(7)-\mathrm{C}(8)-\mathrm{C}(9)-\mathrm{O}(3)$ & $-145.22(18)$ \\
\hline $\mathrm{C}(1)-\mathrm{C}(8)-\mathrm{C}(9)-\mathrm{O}(3)$ & $33.68(18)$ \\
\hline$C(7)-C(8)-C(9)-C(26)$ & $-25.1(3)$ \\
\hline$C(1)-C(8)-C(9)-C(26)$ & $153.82(15)$ \\
\hline$C(7)-C(8)-C(9)-C(32)$ & $98.5(2)$ \\
\hline$C(1)-C(8)-C(9)-C(32)$ & $-82.57(18)$ \\
\hline $\mathrm{C}(3)-\mathrm{N}(1)-\mathrm{C}(10)-\mathrm{C}(11)$ & $-53.1(2)$ \\
\hline $\mathrm{C}(4)-\mathrm{N}(1)-\mathrm{C}(10)-\mathrm{C}(11)$ & $119.4(2)$ \\
\hline $\mathrm{C}(3)-\mathrm{N}(1)-\mathrm{C}(10)-\mathrm{C}(15)$ & $128.8(2)$ \\
\hline$C(4)-N(1)-C(10)-C(15)$ & $-58.7(2)$ \\
\hline$C(15)-C(10)-C(11)-C(12)$ & $0.0(3)$ \\
\hline $\mathrm{N}(1)-\mathrm{C}(10)-\mathrm{C}(11)-\mathrm{C}(12)$ & $-178.06(17)$ \\
\hline$C(10)-C(11)-C(12)-C(13)$ & $-0.2(3)$ \\
\hline$C(11)-C(12)-C(13)-C(14)$ & $0.3(3)$ \\
\hline
\end{tabular}




$\begin{array}{lc}\mathrm{C}(12)-\mathrm{C}(13)-\mathrm{C}(14)-\mathrm{C}(15) & -0.2(3) \\ \mathrm{C}(11)-\mathrm{C}(10)-\mathrm{C}(15)-\mathrm{C}(14) & 0.1(3) \\ \mathrm{N}(1)-\mathrm{C}(10)-\mathrm{C}(15)-\mathrm{C}(14) & 178.16(18) \\ \mathrm{C}(13)-\mathrm{C}(14)-\mathrm{C}(15)-\mathrm{C}(10) & 0.0(3) \\ \mathrm{Si}(2)-\mathrm{O}(4)-\mathrm{C}(16)-\mathrm{C}(6) & -130.19(15) \\ \mathrm{C}(7)-\mathrm{C}(6)-\mathrm{C}(16)-\mathrm{O}(4) & -58.7(2) \\ \mathrm{C}(5)-\mathrm{C}(6)-\mathrm{C}(16)-\mathrm{O}(4) & 177.31(15) \\ \mathrm{C}(23 \mathrm{~B})-\mathrm{Si}(2)-\mathrm{C}(17)-\mathrm{C}(19) & -63.7(6) \\ \mathrm{O}(4)-\mathrm{Si}(2)-\mathrm{C}(17)-\mathrm{C}(19) & 73.4(4) \\ \mathrm{C}(20)-\mathrm{Si}(2)-\mathrm{C}(17)-\mathrm{C}(19) & -159.1(3) \\ \mathrm{C}(17 \mathrm{~B})-\mathrm{Si}(2)-\mathrm{C}(17)-\mathrm{C}(19) & 147(4) \\ \mathrm{C}(23)-\mathrm{Si}(2)-\mathrm{C}(17)-\mathrm{C}(19) & -40.5(4) \\ \mathrm{C}(20 \mathrm{~B})-\mathrm{Si}(2)-\mathrm{C}(17)-\mathrm{C}(19) & 175.0(4) \\ \mathrm{C}(23 \mathrm{~B})-\mathrm{Si}(2)-\mathrm{C}(17)-\mathrm{C}(18) & 172.6(5) \\ \mathrm{O}(4)-\mathrm{Si}(2)-\mathrm{C}(17)-\mathrm{C}(18) & -50.3(4) \\ \mathrm{C}(20)-\mathrm{Si}(2)-\mathrm{C}(17)-\mathrm{C}(18) & 77.2(4) \\ \mathrm{C}(17 \mathrm{~B})-\mathrm{Si}(2)-\mathrm{C}(17)-\mathrm{C}(18) & 24(4) \\ \mathrm{C}(23)-\mathrm{Si}(2)-\mathrm{C}(17)-\mathrm{C}(18) & -164.1(3) \\ \mathrm{C}(20 \mathrm{~B})-\mathrm{Si}(2)-\mathrm{C}(17)-\mathrm{C}(18) & 51.4(4) \\ \mathrm{C}(23 \mathrm{~B})-\mathrm{Si}(2)-\mathrm{C}(20)-\mathrm{C}(22) & -105.1(4) \\ \mathrm{O}(4)-\mathrm{Si}(2)-\mathrm{C}(20)-\mathrm{C}(22) & 135.5(2) \\ \mathrm{C}(17)-\mathrm{Si}(2)-\mathrm{C}(20)-\mathrm{C}(22) & 14.9(3) \\ \mathrm{C}(17 \mathrm{~B})-\mathrm{Si}(2)-\mathrm{C}(20)-\mathrm{C}(22) & 20.3(4) \\ \mathrm{C}(23)-\mathrm{Si}(2)-\mathrm{C}(20)-\mathrm{C}(22) & -104.1(3) \\ \mathrm{C}(20 \mathrm{~B})-\mathrm{Si}(2)-\mathrm{C}(20)-\mathrm{C}(22) & 83.1(6) \\ \mathrm{C}(23 \mathrm{~B})-\mathrm{Si}(2)-\mathrm{C}(20)-\mathrm{C}(21) & 128.0(4) \\ \mathrm{O}(4)-\mathrm{Si}(2)-\mathrm{C}(20)-\mathrm{C}(21) & 8.6(3) \\ \mathrm{C}(17)-\mathrm{Si}(2)-\mathrm{C}(20)-\mathrm{C}(21) & -112.0(3) \\ \mathrm{C}(17 \mathrm{~B})-\mathrm{Si}(2)-\mathrm{C}(20)-\mathrm{C}(21) & -106.6(4) \\ \mathrm{C}(23)-\mathrm{Si}(2)-\mathrm{C}(20)-\mathrm{C}(21) & 129.0(2) \\ \mathrm{C}(20 \mathrm{~B})-\mathrm{Si}(2)-\mathrm{C}(20)-\mathrm{C}(21) & -43.7(5) \\ \mathrm{C}(23 \mathrm{~B})-\mathrm{Si}(2)-\mathrm{C}(23)-\mathrm{C}(24) & -64.5(8) \\ \mathrm{O}(4)-\mathrm{Si}(2)-\mathrm{C}(23)-\mathrm{C}(24) & 58.5(2) \\ \mathrm{C}(20)-\mathrm{Si}(2)-\mathrm{C}(23)-\mathrm{C}(24) & -67.0(2) \\ \mathrm{C}(17)-\mathrm{Si}(2)-\mathrm{C}(23)-\mathrm{C}(24) & \\ & \\ & \end{array}$




\begin{tabular}{|c|c|}
\hline $\mathrm{C}(17 \mathrm{~B})-\mathrm{Si}(2)-\mathrm{C}(23)-\mathrm{C}(24)$ & $169.9(4)$ \\
\hline $\mathrm{C}(20 \mathrm{~B})-\mathrm{Si}(2)-\mathrm{C}(23)-\mathrm{C}(24)$ & $-62.4(4)$ \\
\hline $\mathrm{C}(23 \mathrm{~B})-\mathrm{Si}(2)-\mathrm{C}(23)-\mathrm{C}(25)$ & $61.0(8)$ \\
\hline $\mathrm{O}(4)-\mathrm{Si}(2)-\mathrm{C}(23)-\mathrm{C}(25)$ & $-175.99(18)$ \\
\hline$C(20)-\operatorname{Si}(2)-C(23)-C(25)$ & $58.6(2)$ \\
\hline$C(17)-\operatorname{Si}(2)-C(23)-C(25)$ & $-65.6(3)$ \\
\hline$C(17 B)-\operatorname{Si}(2)-C(23)-C(25)$ & $-64.6(4)$ \\
\hline$C(20 B)-\operatorname{Si}(2)-C(23)-C(25)$ & $63.1(4)$ \\
\hline $\mathrm{C}(23 \mathrm{~B})-\mathrm{Si}(2)-\mathrm{C}(17 \mathrm{~B})-\mathrm{C}(18 \mathrm{~B})$ & $160.4(8)$ \\
\hline $\mathrm{O}(4)-\mathrm{Si}(2)-\mathrm{C}(17 \mathrm{~B})-\mathrm{C}(18 \mathrm{~B})$ & $-59.0(8)$ \\
\hline $\mathrm{C}(20)-\mathrm{Si}(2)-\mathrm{C}(17 \mathrm{~B})-\mathrm{C}(18 \mathrm{~B})$ & $65.1(8)$ \\
\hline $\mathrm{C}(17)-\mathrm{Si}(2)-\mathrm{C}(17 \mathrm{~B})-\mathrm{C}(18 \mathrm{~B})$ & $-166(5)$ \\
\hline $\mathrm{C}(23)-\mathrm{Si}(2)-\mathrm{C}(17 \mathrm{~B})-\mathrm{C}(18 \mathrm{~B})$ & $-174.5(7)$ \\
\hline $\mathrm{C}(20 \mathrm{~B})-\mathrm{Si}(2)-\mathrm{C}(17 \mathrm{~B})-\mathrm{C}(18 \mathrm{~B})$ & $40.9(8)$ \\
\hline $\mathrm{C}(23 \mathrm{~B})-\mathrm{Si}(2)-\mathrm{C}(17 \mathrm{~B})-\mathrm{C}(19 \mathrm{~B})$ & $-74.6(8)$ \\
\hline $\mathrm{O}(4)-\mathrm{Si}(2)-\mathrm{C}(17 \mathrm{~B})-\mathrm{C}(19 \mathrm{~B})$ & $65.9(6)$ \\
\hline $\mathrm{C}(20)-\mathrm{Si}(2)-\mathrm{C}(17 \mathrm{~B})-\mathrm{C}(19 \mathrm{~B})$ & $-170.0(5)$ \\
\hline $\mathrm{C}(17)-\mathrm{Si}(2)-\mathrm{C}(17 \mathrm{~B})-\mathrm{C}(19 \mathrm{~B})$ & $-41(4)$ \\
\hline $\mathrm{C}(23)-\mathrm{Si}(2)-\mathrm{C}(17 \mathrm{~B})-\mathrm{C}(19 \mathrm{~B})$ & $-49.6(7)$ \\
\hline$C(20 B)-S i(2)-C(17 B)-C(19 B)$ & $165.8(6)$ \\
\hline $\mathrm{C}(23 \mathrm{~B})-\mathrm{Si}(2)-\mathrm{C}(20 \mathrm{~B})-\mathrm{C}(22 \mathrm{~B})$ & $-73.9(7)$ \\
\hline $\mathrm{O}(4)-\mathrm{Si}(2)-\mathrm{C}(20 \mathrm{~B})-\mathrm{C}(22 \mathrm{~B})$ & $160.6(6)$ \\
\hline $\mathrm{C}(20)-\mathrm{Si}(2)-\mathrm{C}(20 \mathrm{~B})-\mathrm{C}(22 \mathrm{~B})$ & $-65.3(7)$ \\
\hline $\mathrm{C}(17)-\mathrm{Si}(2)-\mathrm{C}(20 \mathrm{~B})-\mathrm{C}(22 \mathrm{~B})$ & $55.2(7)$ \\
\hline$C(17 B)-S i(2)-C(20 B)-C(22 B)$ & $58.1(7)$ \\
\hline $\mathrm{C}(23)-\mathrm{Si}(2)-\mathrm{C}(20 \mathrm{~B})-\mathrm{C}(22 \mathrm{~B})$ & $-74.8(7)$ \\
\hline$C(23 B)-S i(2)-C(20 B)-C(21 B)$ & $52.9(7)$ \\
\hline $\mathrm{O}(4)-\mathrm{Si}(2)-\mathrm{C}(20 \mathrm{~B})-\mathrm{C}(21 \mathrm{~B})$ & $-72.5(6)$ \\
\hline $\mathrm{C}(20)-\mathrm{Si}(2)-\mathrm{C}(20 \mathrm{~B})-\mathrm{C}(21 \mathrm{~B})$ & $61.5(6)$ \\
\hline$C(17)-\operatorname{Si}(2)-C(20 B)-C(21 B)$ & $-178.0(6)$ \\
\hline$C(17 B)-S i(2)-C(20 B)-C(21 B)$ & $-175.1(6)$ \\
\hline $\mathrm{C}(23)-\mathrm{Si}(2)-\mathrm{C}(20 \mathrm{~B})-\mathrm{C}(21 \mathrm{~B})$ & $52.0(7)$ \\
\hline $\mathrm{O}(4)-\mathrm{Si}(2)-\mathrm{C}(23 \mathrm{~B})-\mathrm{C}(24 \mathrm{~B})$ & $-31.3(9)$ \\
\hline $\mathrm{C}(20)-\mathrm{Si}(2)-\mathrm{C}(23 \mathrm{~B})-\mathrm{C}(24 \mathrm{~B})$ & $-146.7(8)$ \\
\hline $\mathrm{C}(17)-\mathrm{Si}(2)-\mathrm{C}(23 \mathrm{~B})-\mathrm{C}(24 \mathrm{~B})$ & $98.7(7)$ \\
\hline
\end{tabular}




\begin{tabular}{|c|c|}
\hline$C(17 B)-S i(2)-C(23 B)-C(24 B)$ & $102.7(8)$ \\
\hline $\mathrm{C}(23)-\mathrm{Si}(2)-\mathrm{C}(23 \mathrm{~B})-\mathrm{C}(24 \mathrm{~B})$ & $35.6(7)$ \\
\hline$C(20 B)-S i(2)-C(23 B)-C(24 B)$ & $-142.7(7)$ \\
\hline $\mathrm{O}(4)-\mathrm{Si}(2)-\mathrm{C}(23 \mathrm{~B})-\mathrm{C}(25 \mathrm{~B})$ & $-157.4(6)$ \\
\hline $\mathrm{C}(20)-\mathrm{Si}(2)-\mathrm{C}(23 \mathrm{~B})-\mathrm{C}(25 \mathrm{~B})$ & $87.2(7)$ \\
\hline $\mathrm{C}(17)-\mathrm{Si}(2)-\mathrm{C}(23 \mathrm{~B})-\mathrm{C}(25 \mathrm{~B})$ & $-27.4(9)$ \\
\hline$C(17 B)-S i(2)-C(23 B)-C(25 B)$ & $-23.4(10)$ \\
\hline $\mathrm{C}(23)-\mathrm{Si}(2)-\mathrm{C}(23 \mathrm{~B})-\mathrm{C}(25 \mathrm{~B})$ & $-90.4(10)$ \\
\hline$C(20 B)-S i(2)-C(23 B)-C(25 B)$ & $91.2(8)$ \\
\hline $\mathrm{O}(3)-\mathrm{C}(9)-\mathrm{C}(26)-\mathrm{C}(31)$ & $8.3(2)$ \\
\hline $\mathrm{C}(32)-\mathrm{C}(9)-\mathrm{C}(26)-\mathrm{C}(31)$ & $126.15(19)$ \\
\hline $\mathrm{C}(8)-\mathrm{C}(9)-\mathrm{C}(26)-\mathrm{C}(31)$ & $-110.3(2)$ \\
\hline $\mathrm{O}(3)-\mathrm{C}(9)-\mathrm{C}(26)-\mathrm{C}(27)$ & $-171.35(16)$ \\
\hline $\mathrm{C}(32)-\mathrm{C}(9)-\mathrm{C}(26)-\mathrm{C}(27)$ & $-53.5(2)$ \\
\hline $\mathrm{C}(8)-\mathrm{C}(9)-\mathrm{C}(26)-\mathrm{C}(27)$ & $70.0(2)$ \\
\hline $\mathrm{C}(31)-\mathrm{C}(26)-\mathrm{C}(27)-\mathrm{C}(28)$ & $2.8(3)$ \\
\hline $\mathrm{C}(9)-\mathrm{C}(26)-\mathrm{C}(27)-\mathrm{C}(28)$ & $-177.49(18)$ \\
\hline $\mathrm{C}(26)-\mathrm{C}(27)-\mathrm{C}(28)-\mathrm{C}(29)$ & $-1.8(3)$ \\
\hline $\mathrm{C}(27)-\mathrm{C}(28)-\mathrm{C}(29)-\mathrm{C}(30)$ & $-0.5(3)$ \\
\hline $\mathrm{C}(28)-\mathrm{C}(29)-\mathrm{C}(30)-\mathrm{C}(31)$ & $1.5(3)$ \\
\hline $\mathrm{C}(27)-\mathrm{C}(26)-\mathrm{C}(31)-\mathrm{C}(30)$ & $-1.8(3)$ \\
\hline $\mathrm{C}(9)-\mathrm{C}(26)-\mathrm{C}(31)-\mathrm{C}(30)$ & $178.58(19)$ \\
\hline $\mathrm{C}(29)-\mathrm{C}(30)-\mathrm{C}(31)-\mathrm{C}(26)$ & $-0.4(3)$ \\
\hline $\mathrm{O}(3)-\mathrm{Si}(1)-\mathrm{C}(33)-\mathrm{C}(34)$ & $-76.90(16)$ \\
\hline $\mathrm{C}(1)-\mathrm{Si}(1)-\mathrm{C}(33)-\mathrm{C}(34)$ & $179.91(15)$ \\
\hline $\mathrm{C}(37)-\mathrm{Si}(1)-\mathrm{C}(33)-\mathrm{C}(34)$ & $45.71(18)$ \\
\hline $\mathrm{O}(3)-\mathrm{Si}(1)-\mathrm{C}(33)-\mathrm{C}(35)$ & $43.42(17)$ \\
\hline $\mathrm{C}(1)-\mathrm{Si}(1)-\mathrm{C}(33)-\mathrm{C}(35)$ & $-59.77(17)$ \\
\hline $\mathrm{C}(37)-\mathrm{Si}(1)-\mathrm{C}(33)-\mathrm{C}(35)$ & $166.03(14)$ \\
\hline $\mathrm{O}(3)-\mathrm{Si}(1)-\mathrm{C}(33)-\mathrm{C}(36)$ & $161.82(14)$ \\
\hline $\mathrm{C}(1)-\mathrm{Si}(1)-\mathrm{C}(33)-\mathrm{C}(36)$ & $58.63(16)$ \\
\hline $\mathrm{C}(37)-\mathrm{Si}(1)-\mathrm{C}(33)-\mathrm{C}(36)$ & $-75.57(17)$ \\
\hline $\mathrm{O}(3)-\mathrm{Si}(1)-\mathrm{C}(37)-\mathrm{C}(38)$ & $174.49(15)$ \\
\hline $\mathrm{C}(33)-\mathrm{Si}(1)-\mathrm{C}(37)-\mathrm{C}(38)$ & $50.09(19)$ \\
\hline $\mathrm{C}(1)-\mathrm{Si}(1)-\mathrm{C}(37)-\mathrm{C}(38)$ & $-81.76(17)$ \\
\hline
\end{tabular}




$\begin{array}{lc}\mathrm{O}(3)-\mathrm{Si}(1)-\mathrm{C}(37)-\mathrm{C}(40) & -64.58(15) \\ \mathrm{C}(33)-\mathrm{Si}(1)-\mathrm{C}(37)-\mathrm{C}(40) & 171.03(13) \\ \mathrm{C}(1)-\mathrm{Si}(1)-\mathrm{C}(37)-\mathrm{C}(40) & 39.17(17) \\ \mathrm{O}(3)-\mathrm{Si}(1)-\mathrm{C}(37)-\mathrm{C}(39) & 52.31(15) \\ \mathrm{C}(33)-\mathrm{Si}(1)-\mathrm{C}(37)-\mathrm{C}(39) & -72.08(16) \\ \mathrm{C}(1)-\mathrm{Si}(1)-\mathrm{C}(37)-\mathrm{C}(39) & 156.07(13)\end{array}$




\section{Spectra of ${ }^{1} \mathrm{H}$ NMR Monitored Reactions}
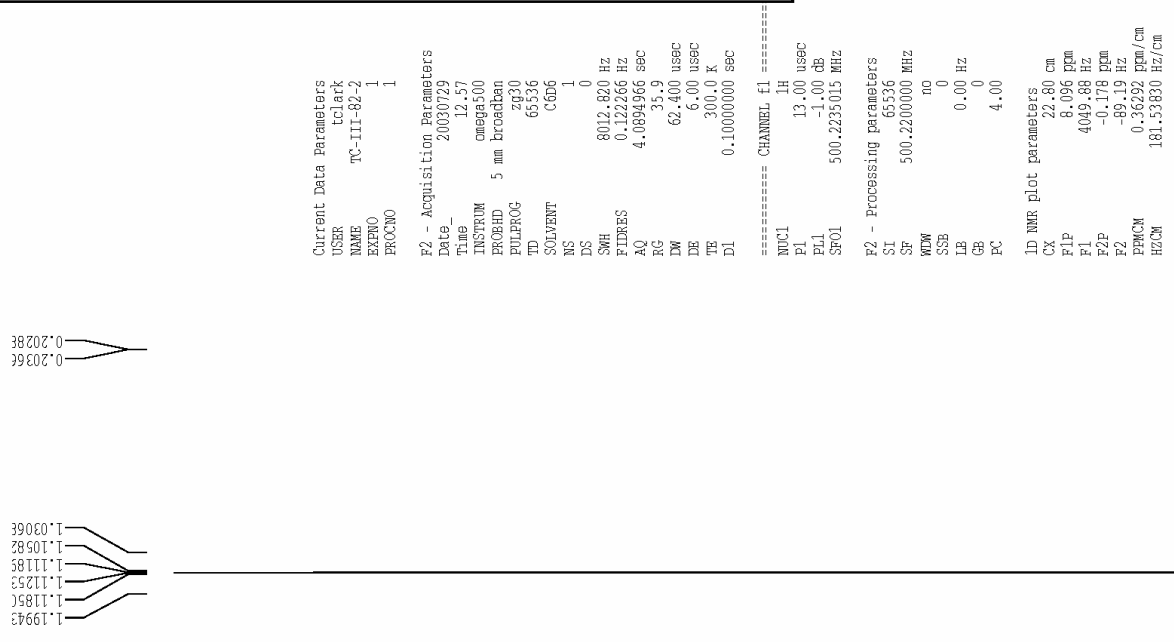

]ETTT:
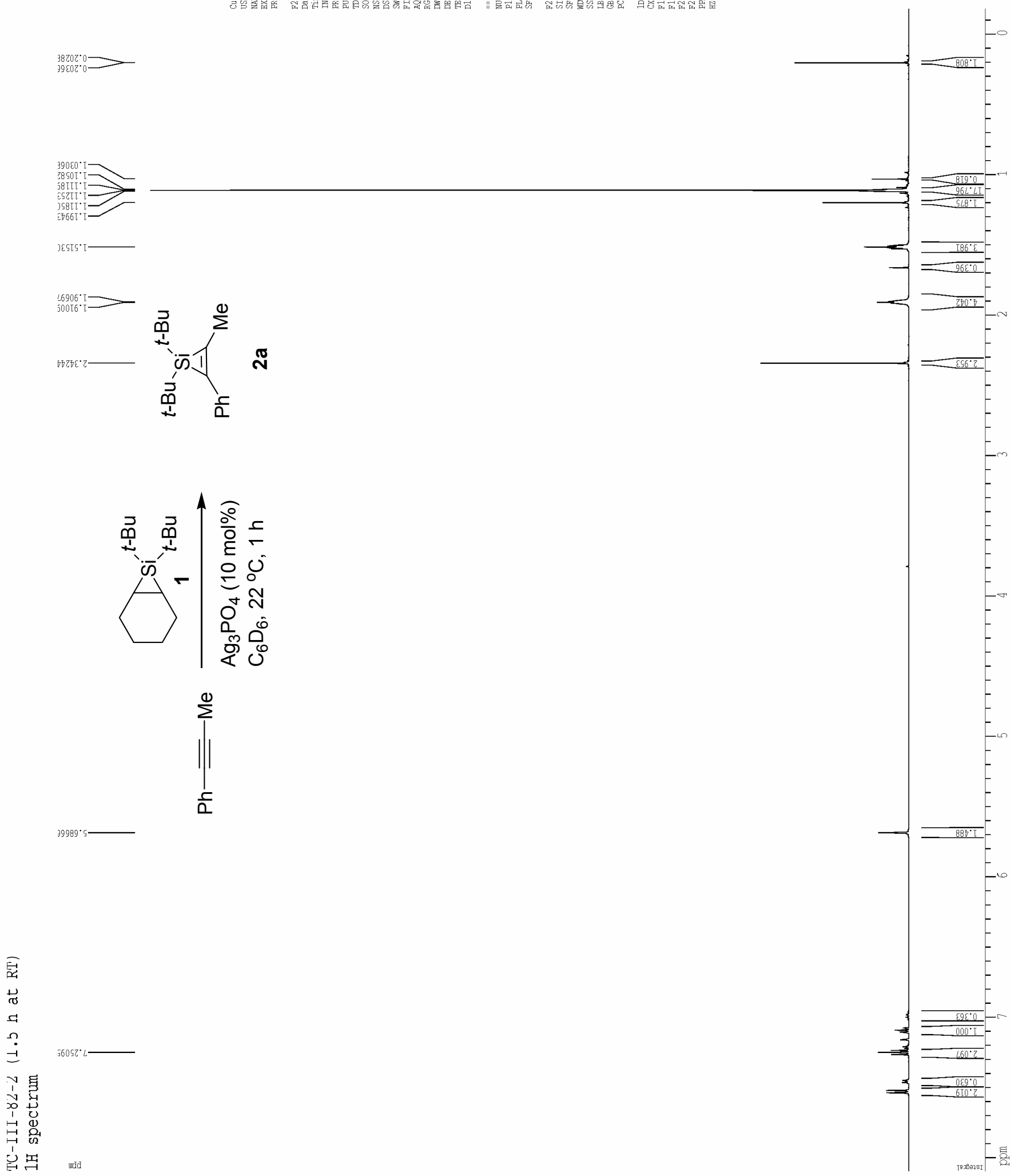


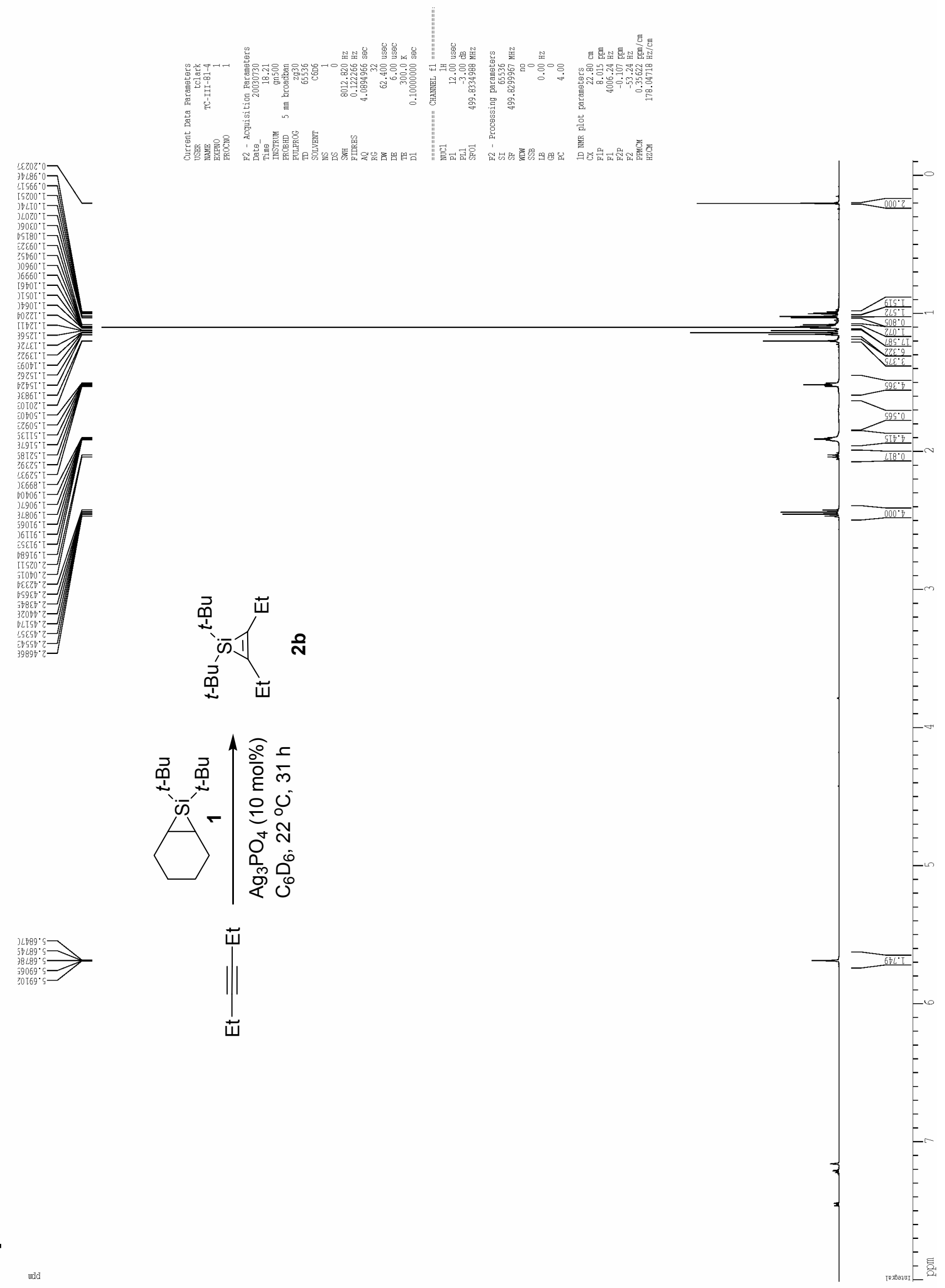



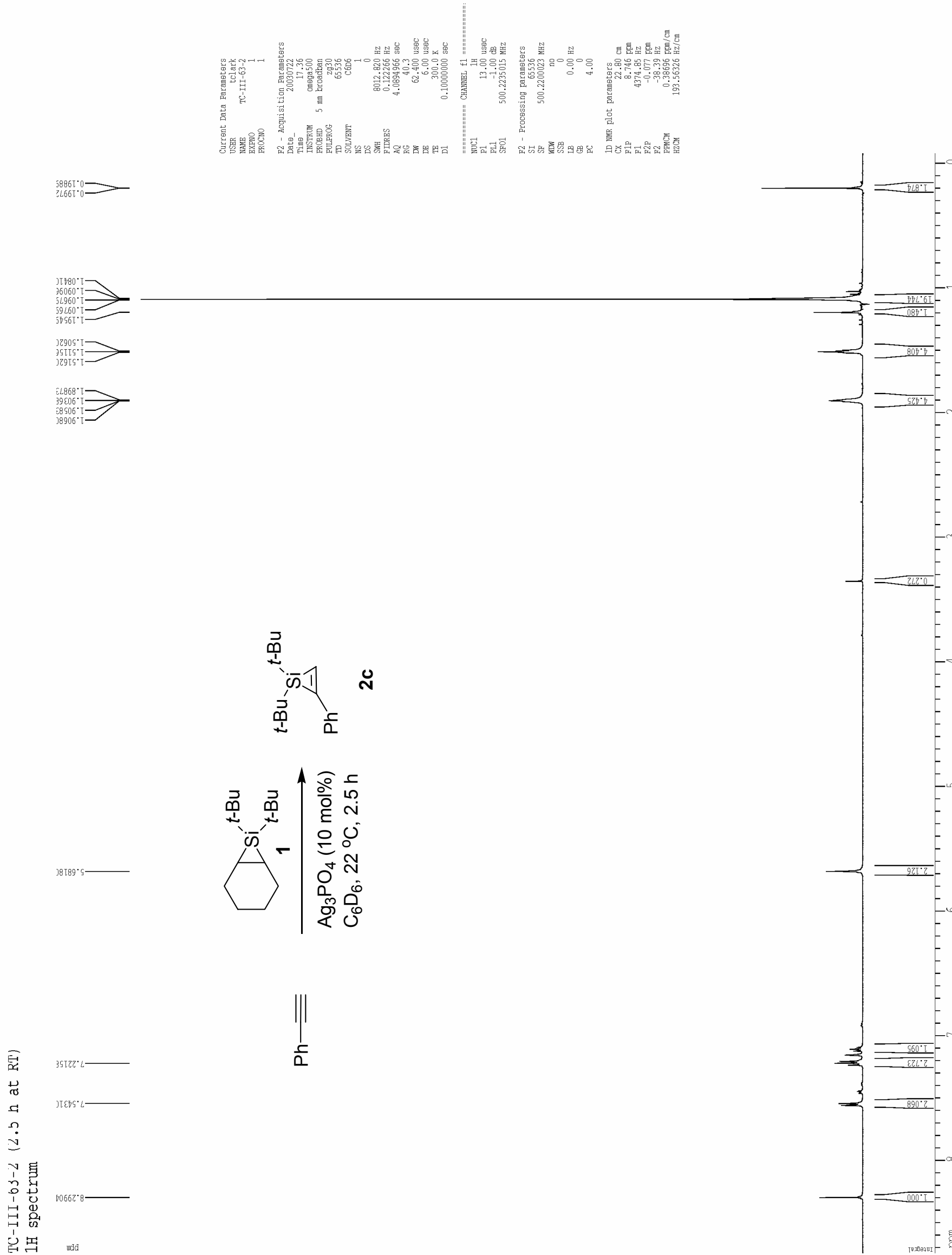

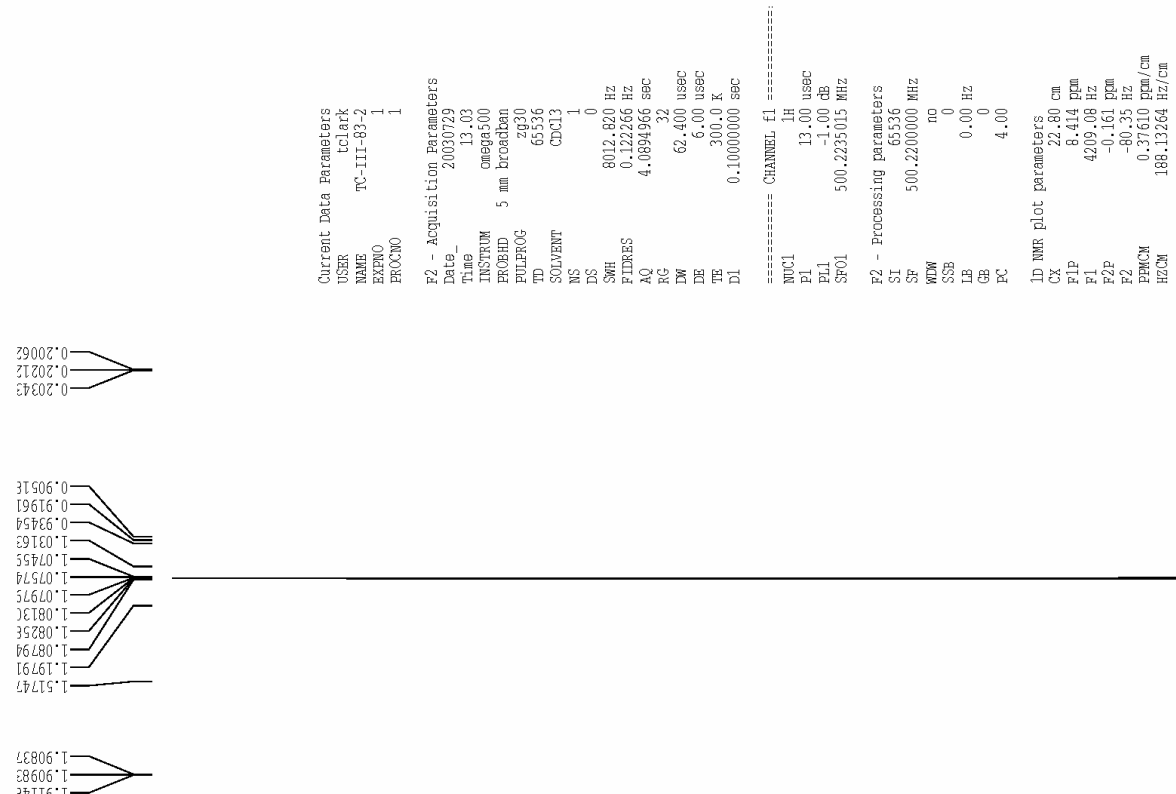

$\stackrel{乛}{9}$

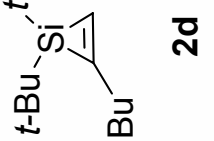

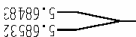
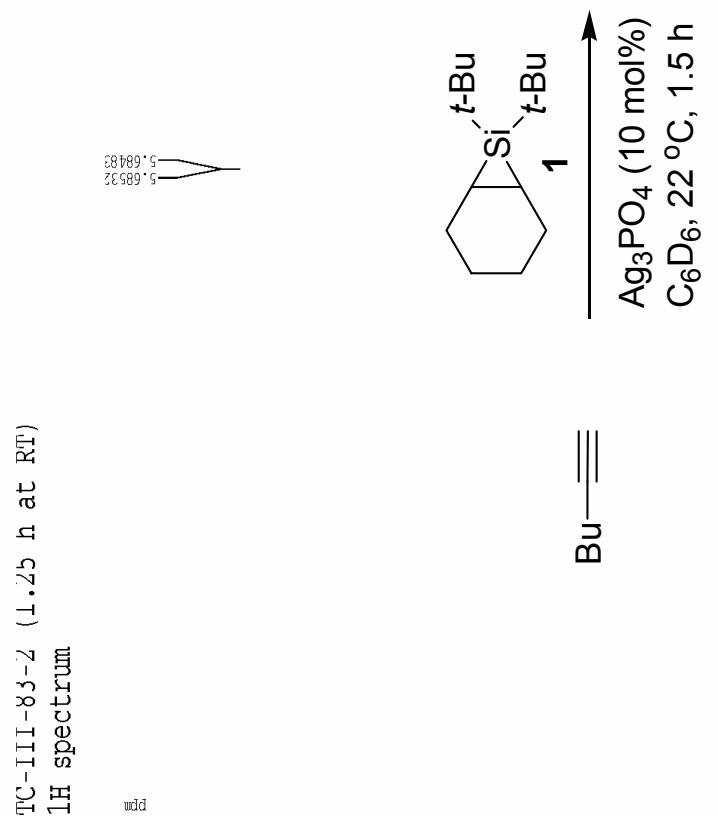

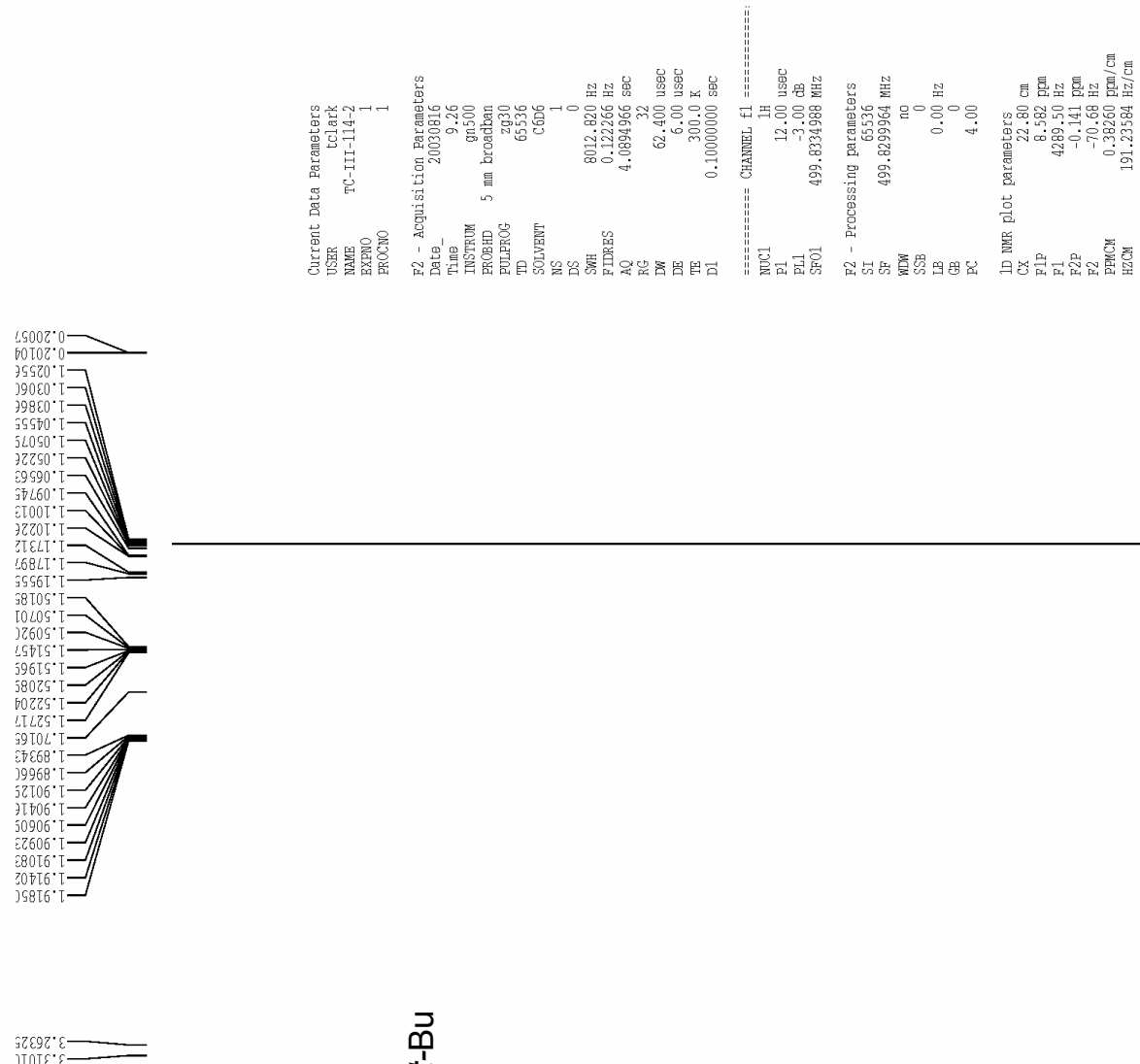

ฉ্
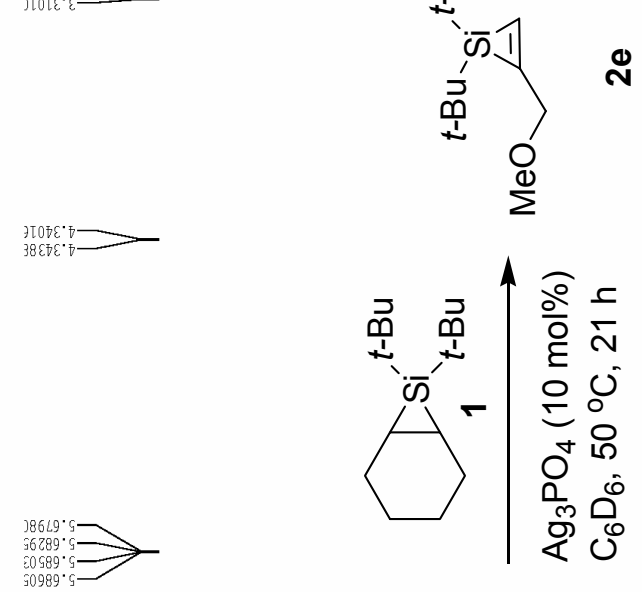

$50589^{\circ} 5$

ง

$\|\left.\right|_{\substack{0 \\ \Sigma}}$

$50090^{\circ}\llcorner-$
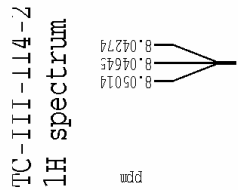

(1)

용

总焉 wad 

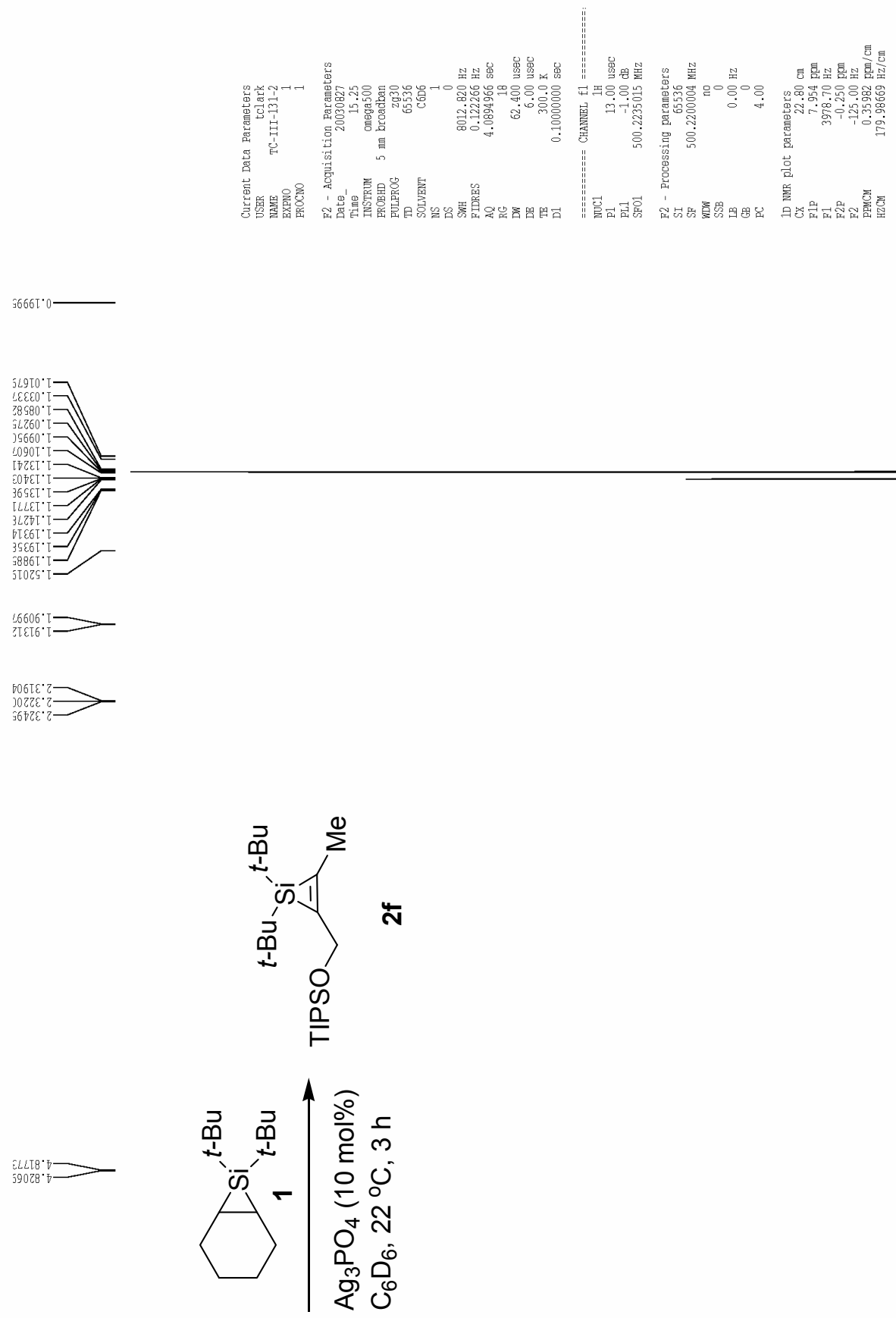

$36669^{\circ} \div-$

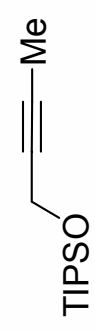

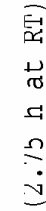
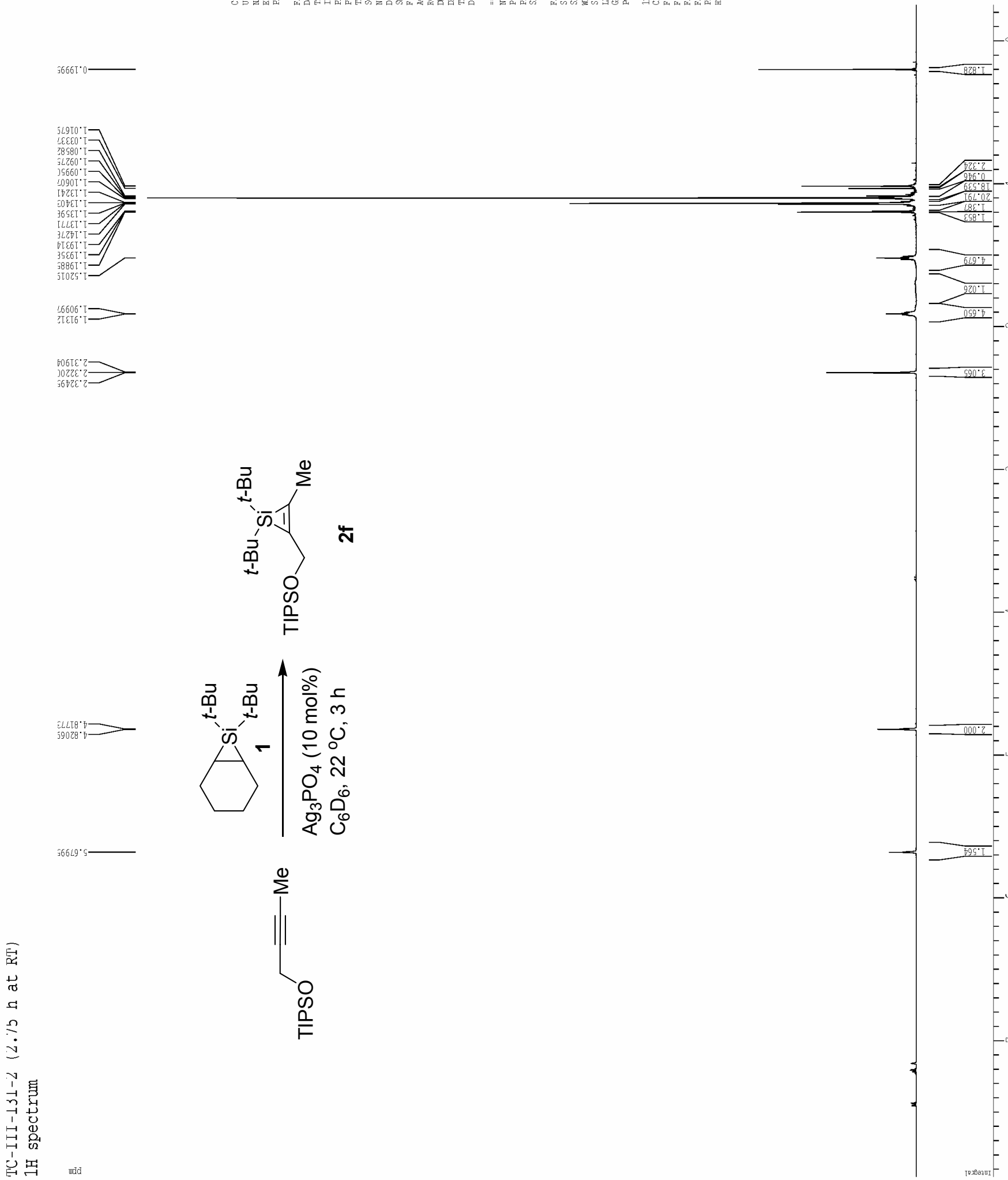

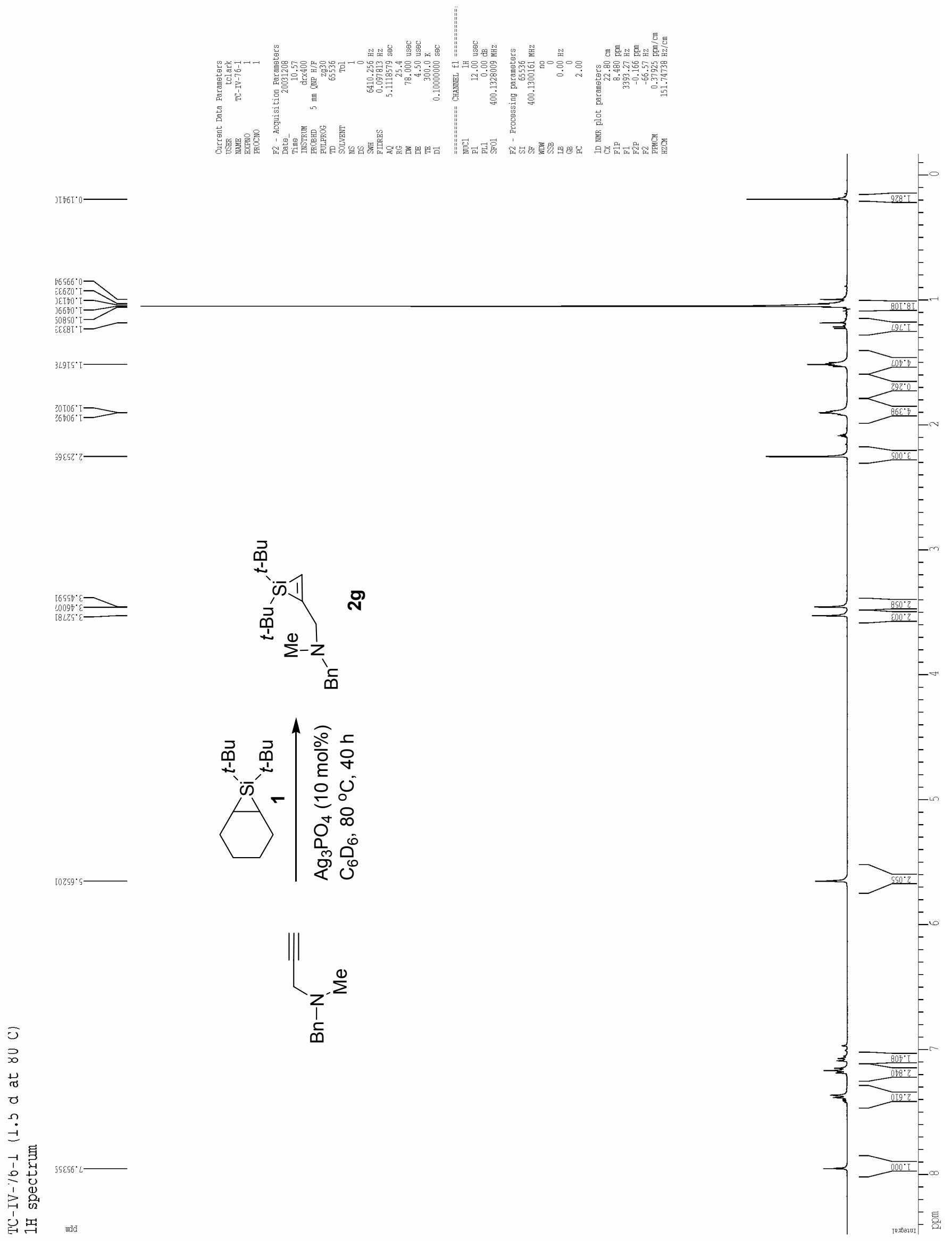

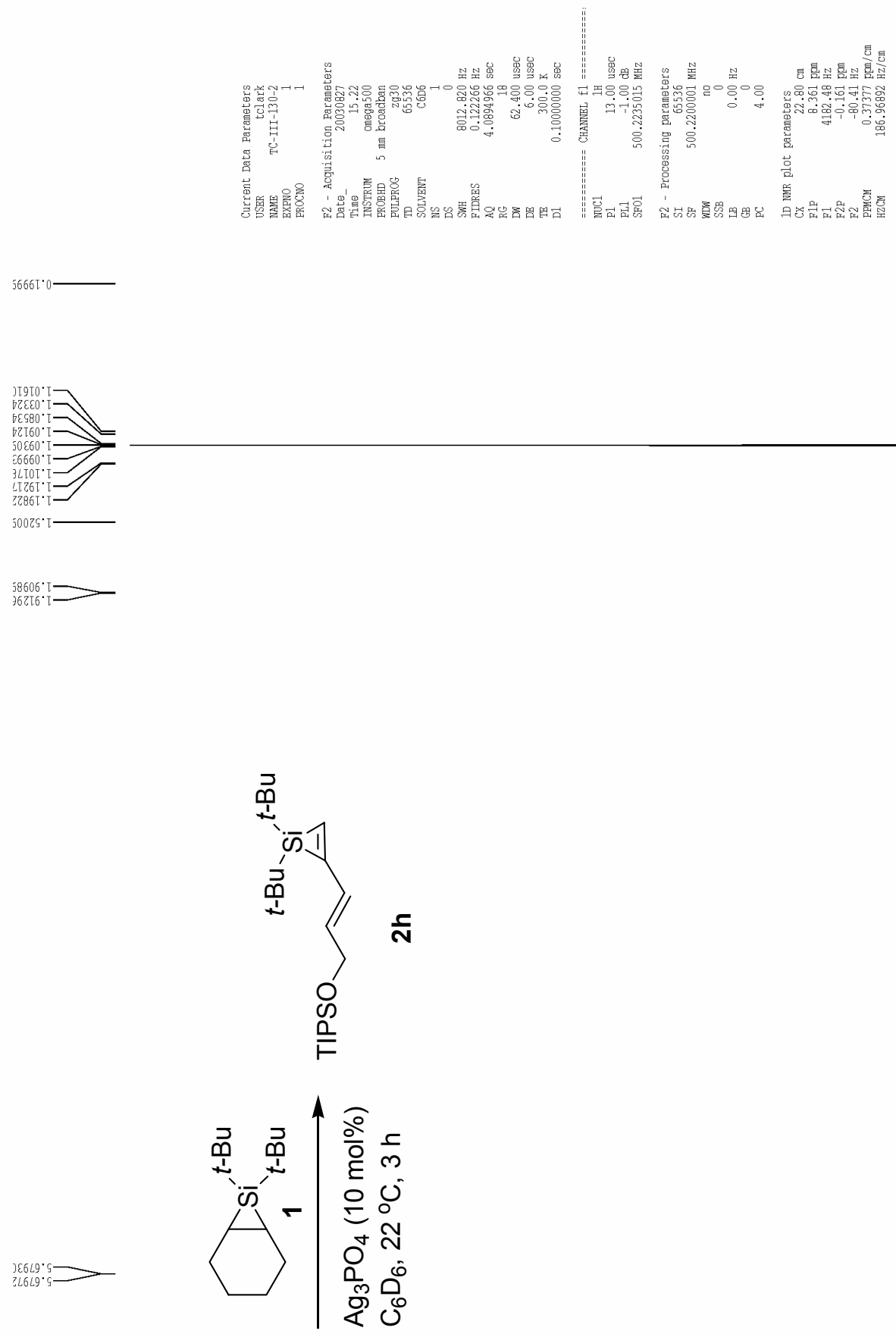

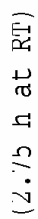

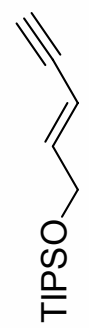

广ै

号㝵

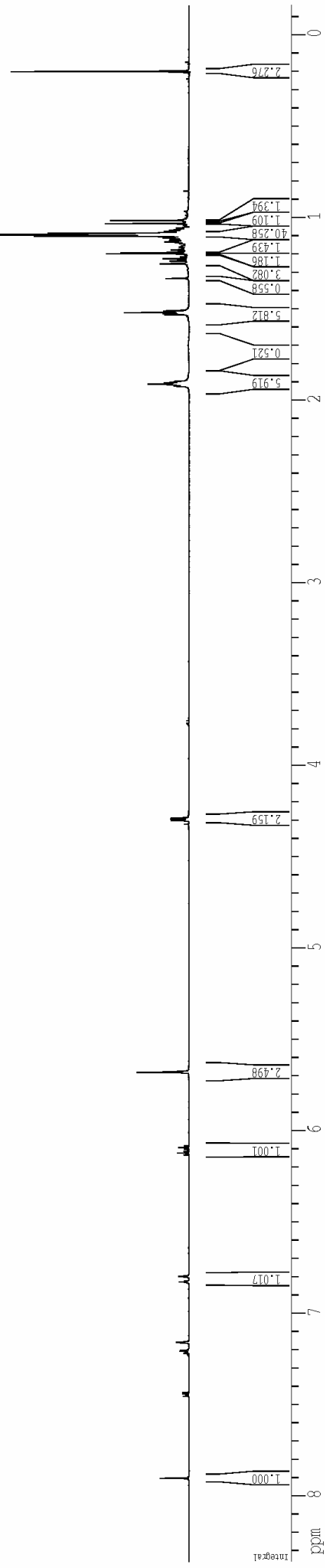




\section{Selected Spectra}
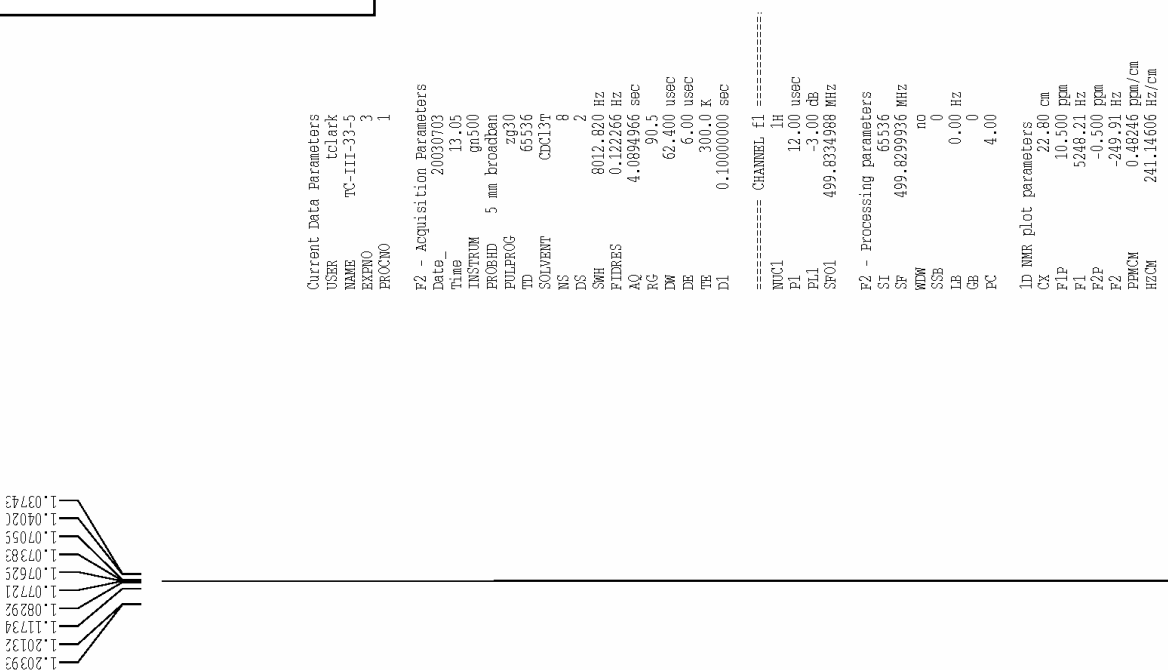

$30<87^{\circ} 7$

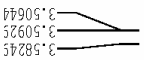

$\stackrel{9}{+}$
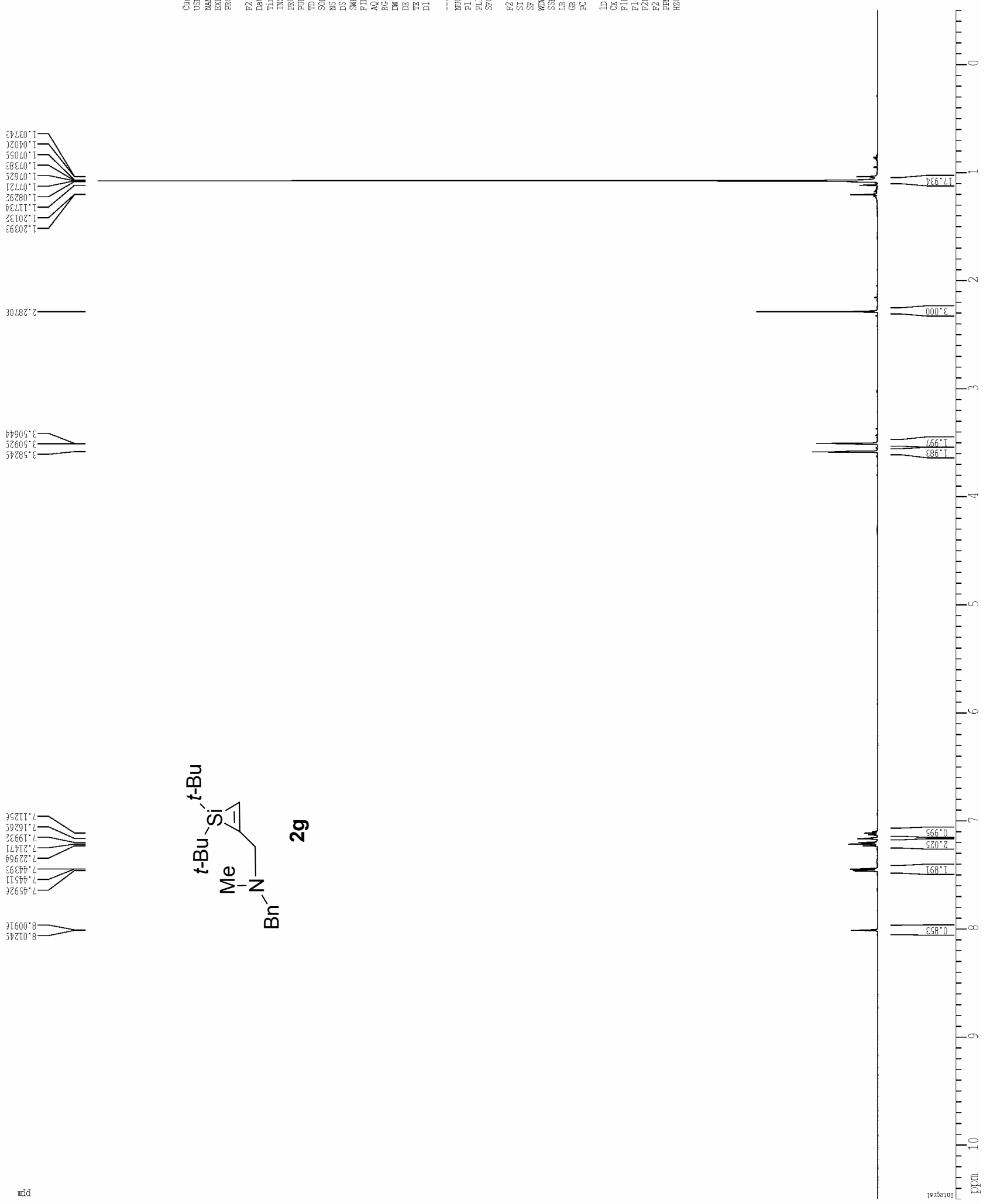

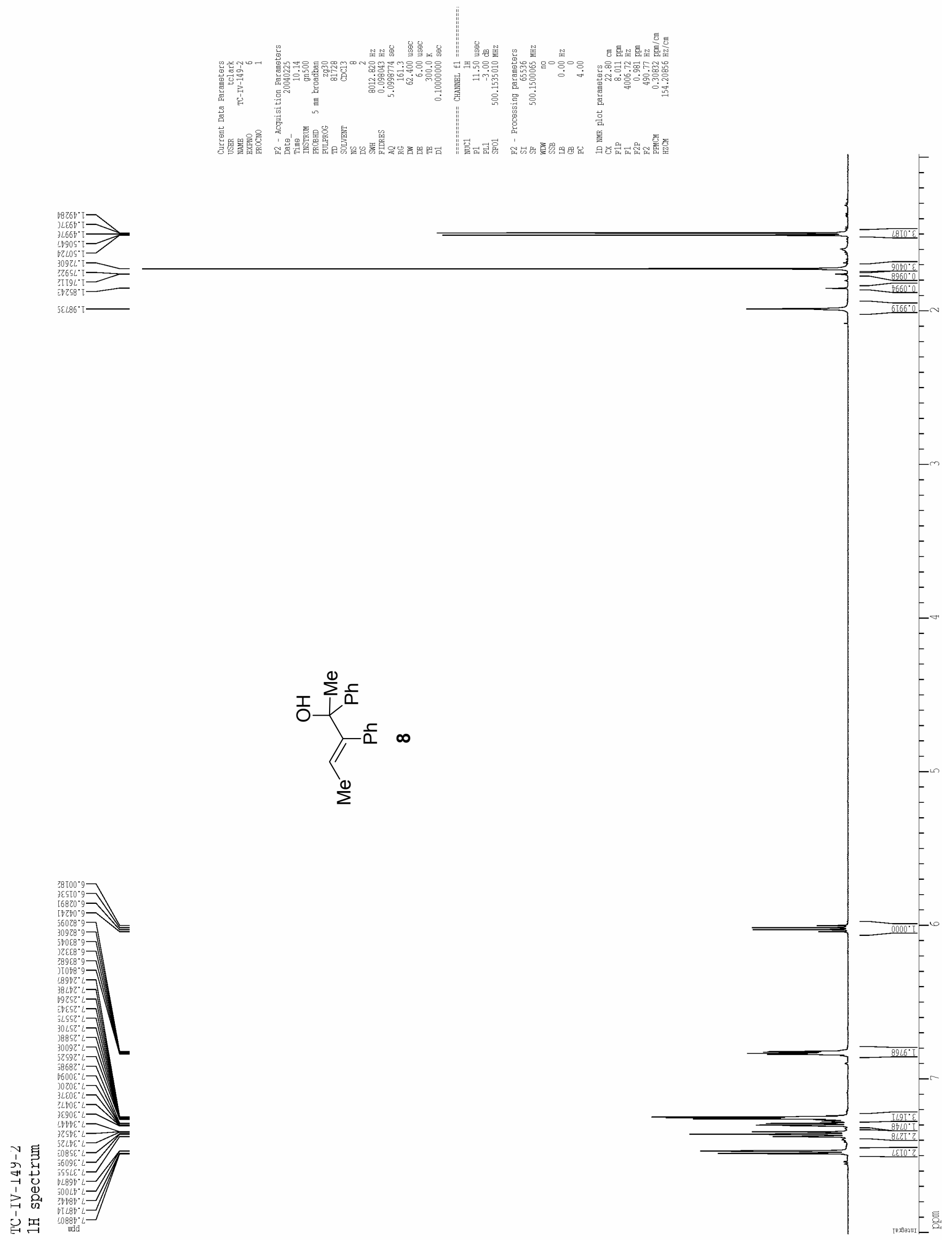

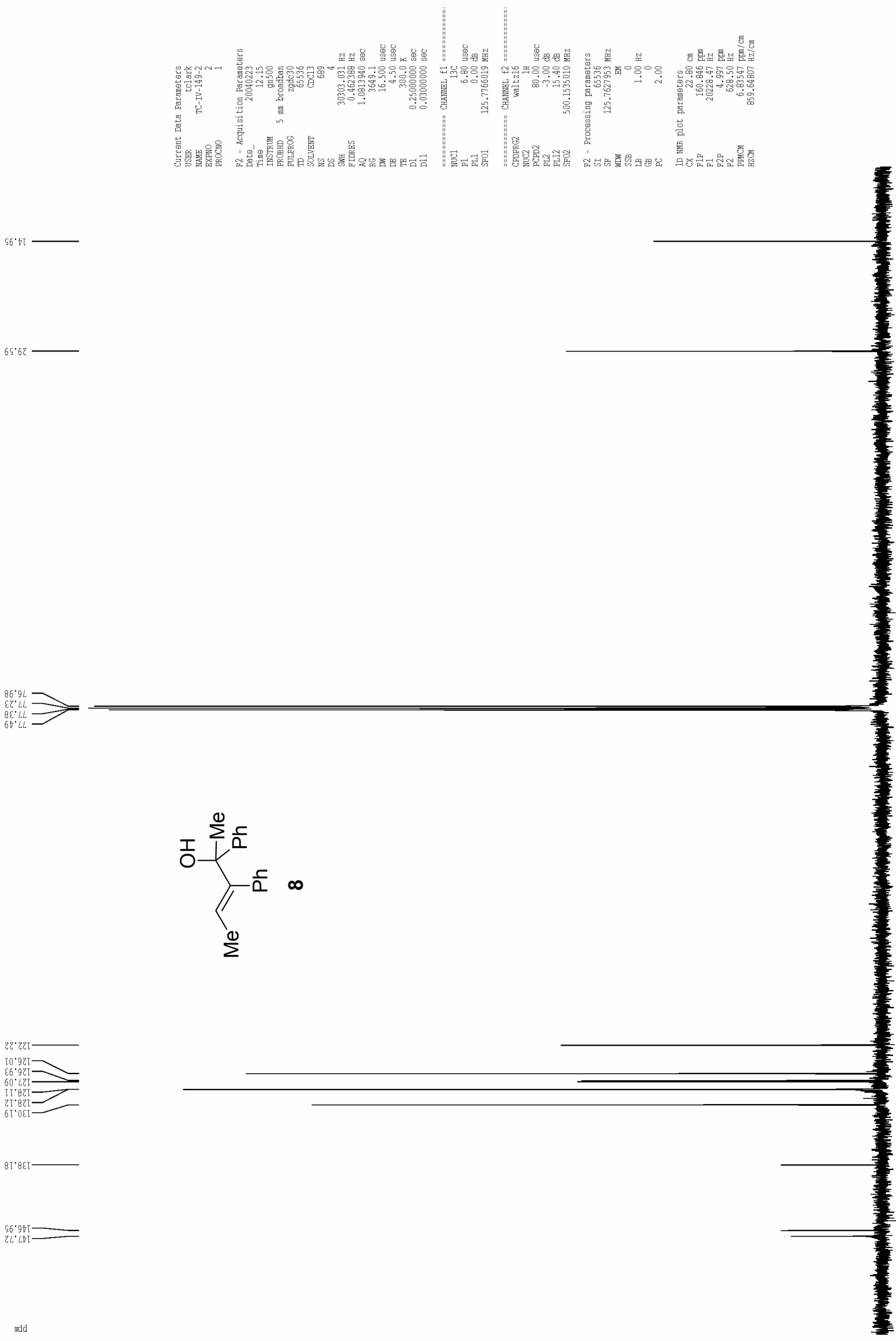

8T: $8 \varepsilon \tau$

章

곤

${ }^{96 \cdot 961 二=}$

गु

1
$>$

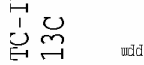



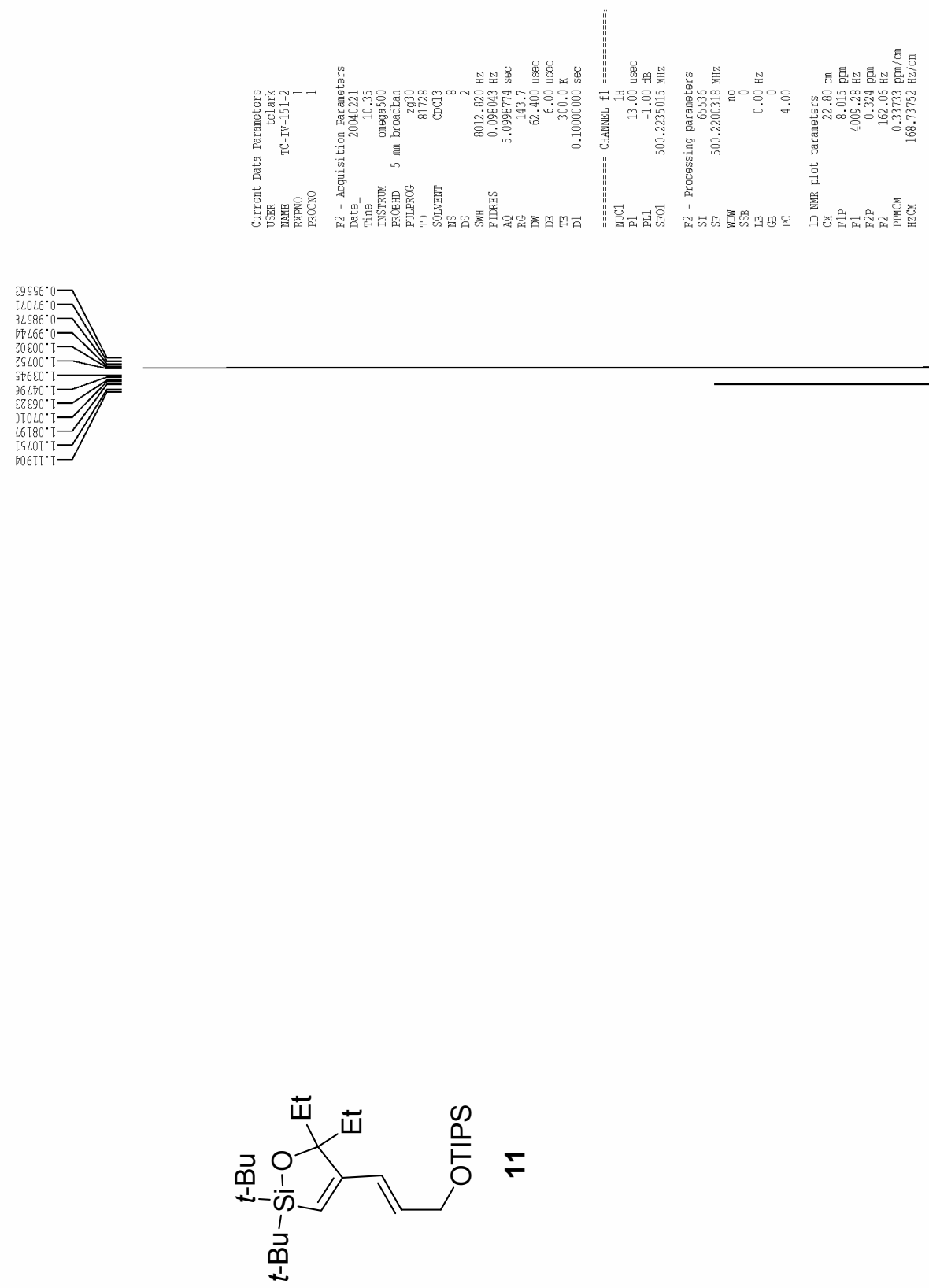

$39998^{\circ} \mathrm{G}$

i 罗

곡뉴

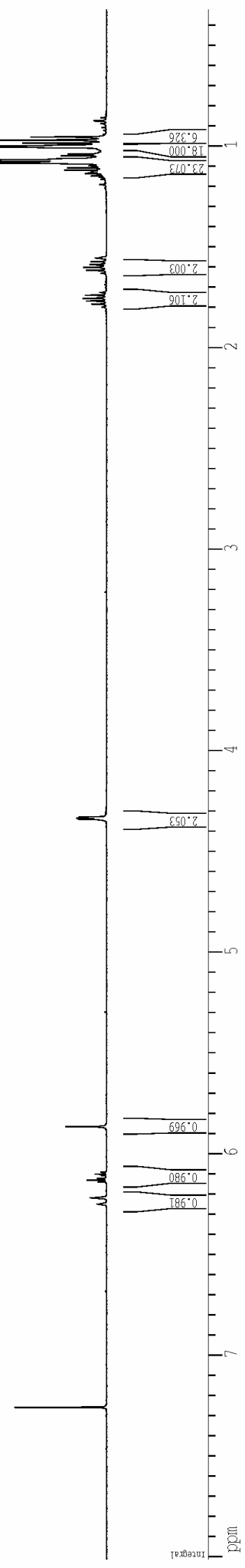




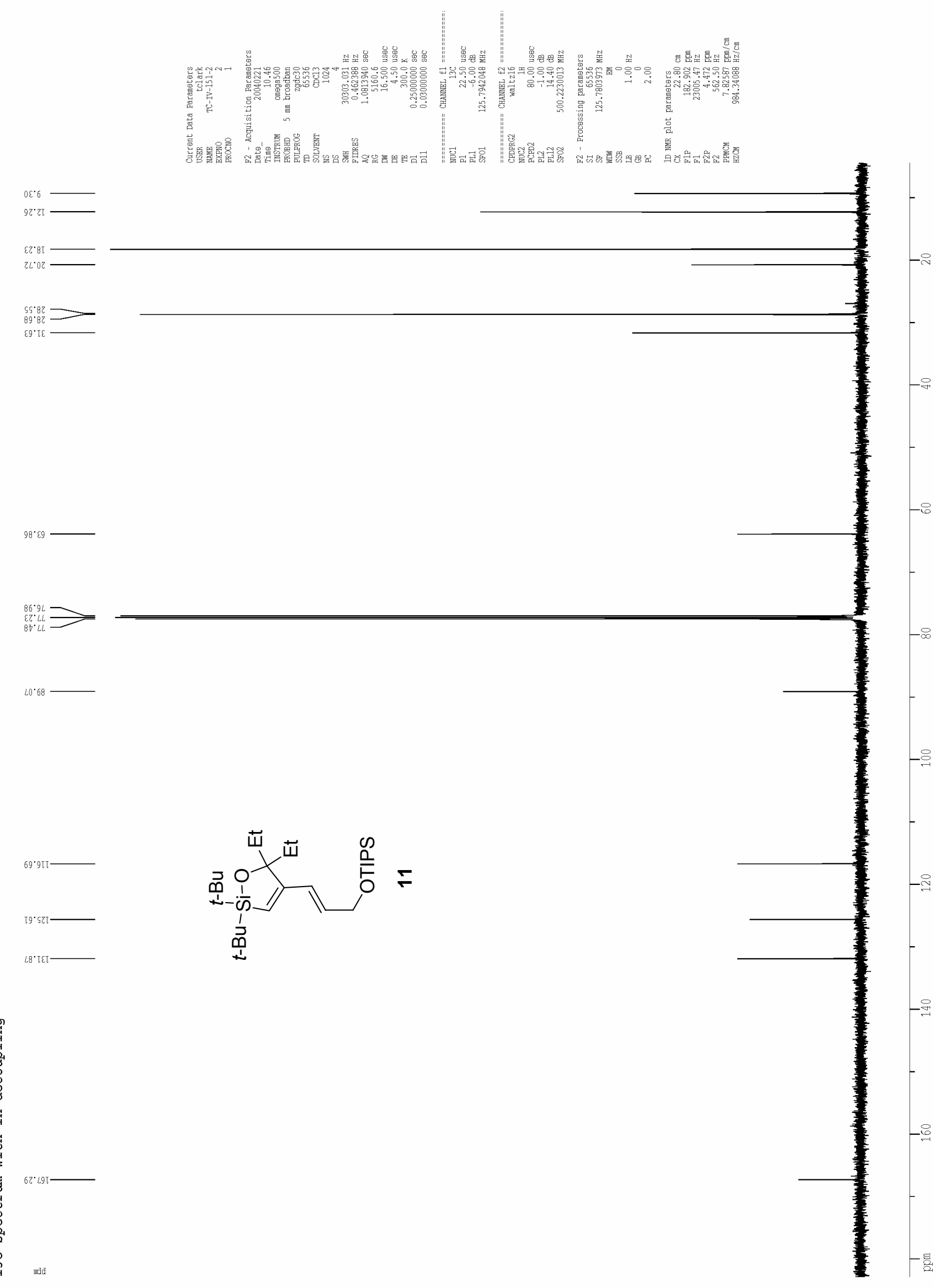



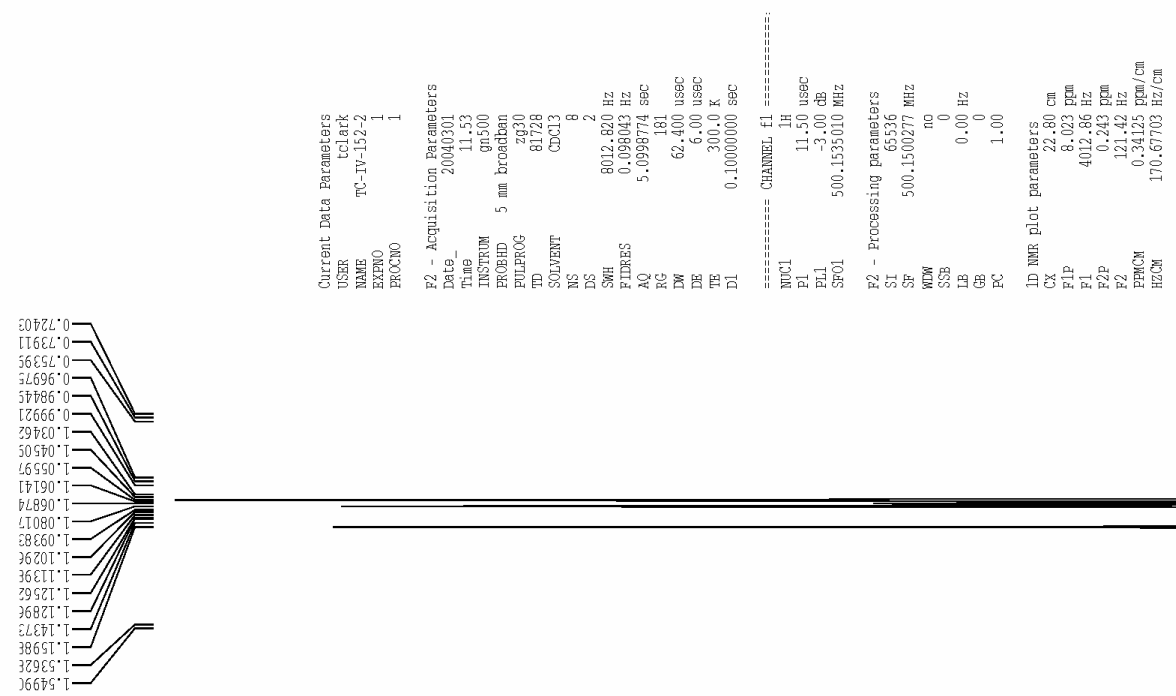

$10987^{\circ} 8-$
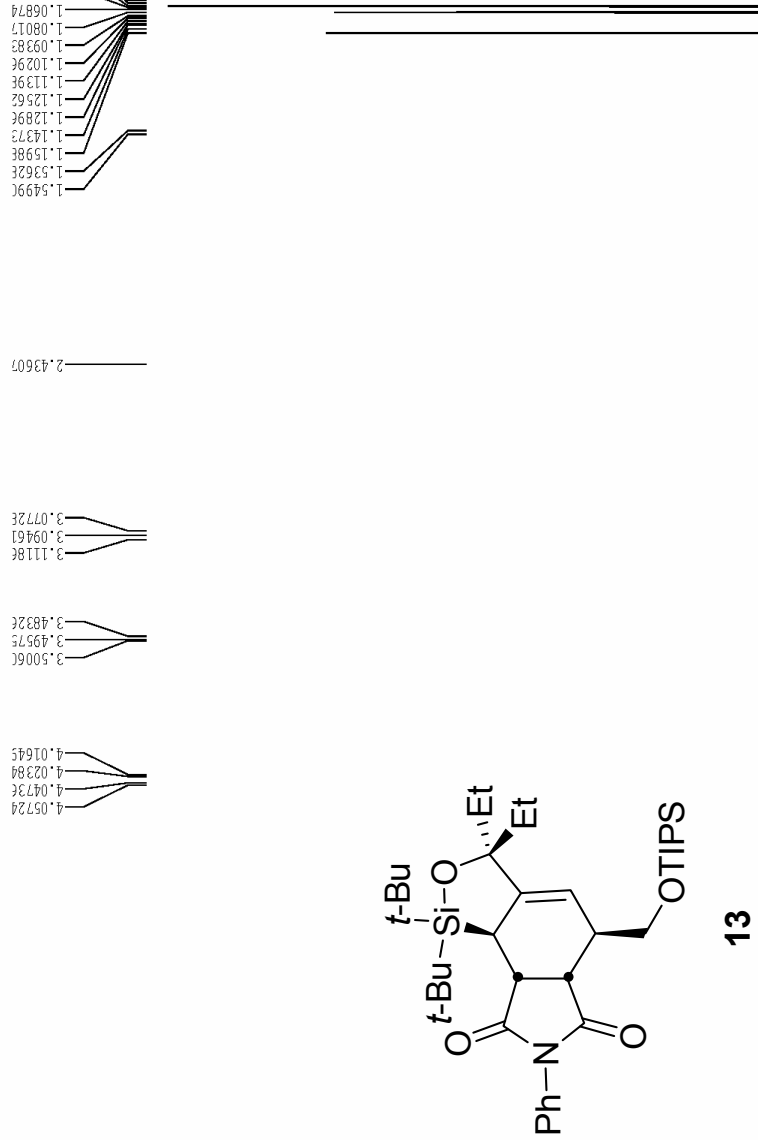

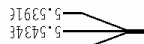

a
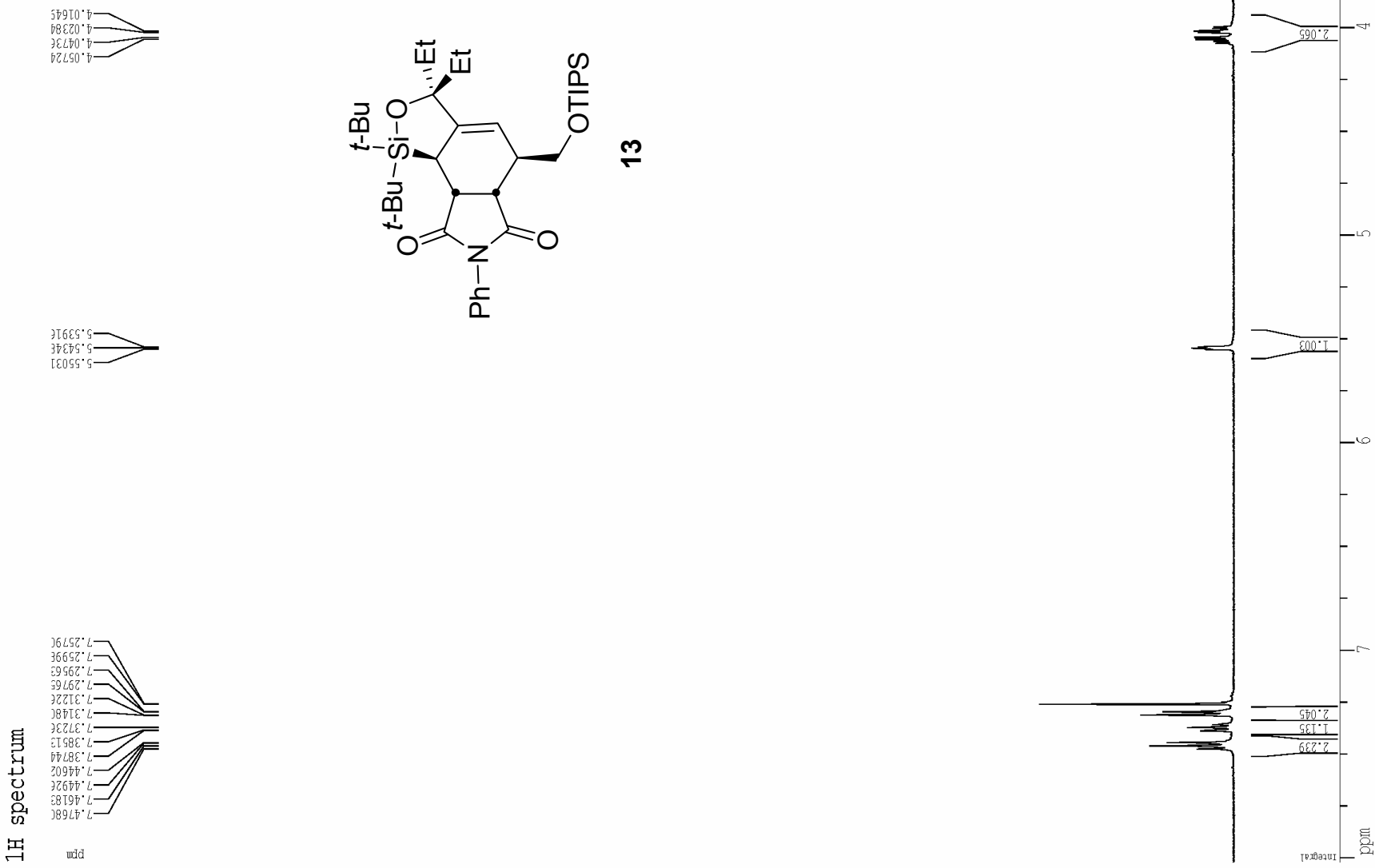

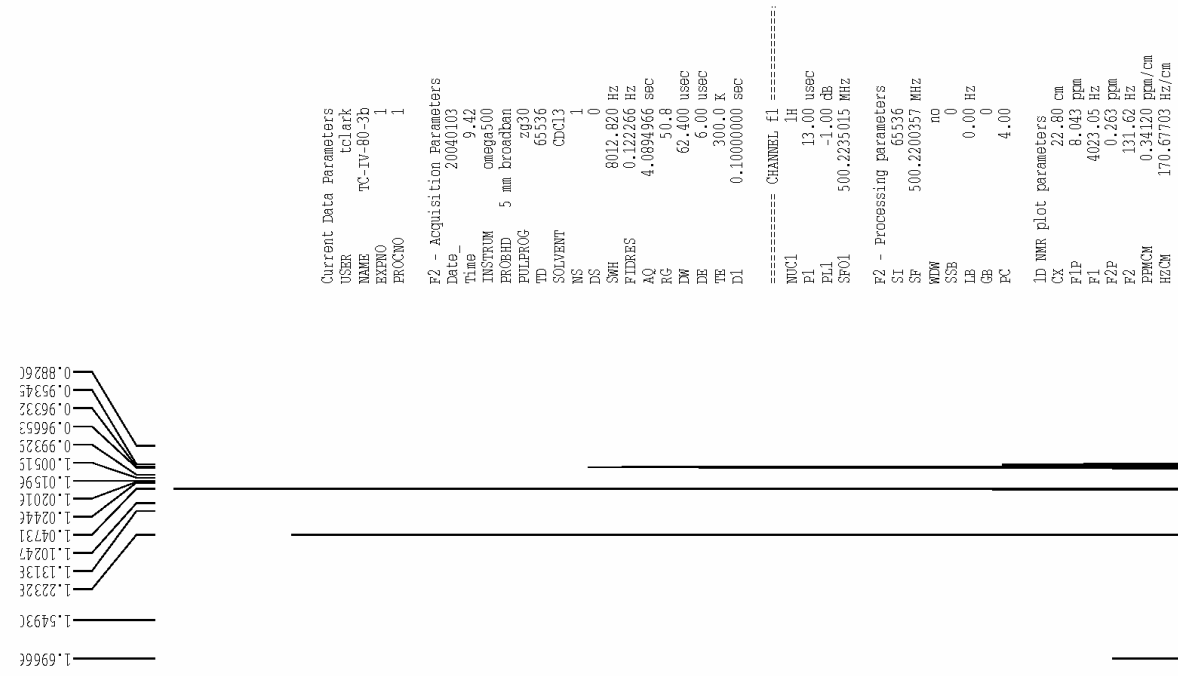

ธъยह ${ }^{*} \varepsilon-$
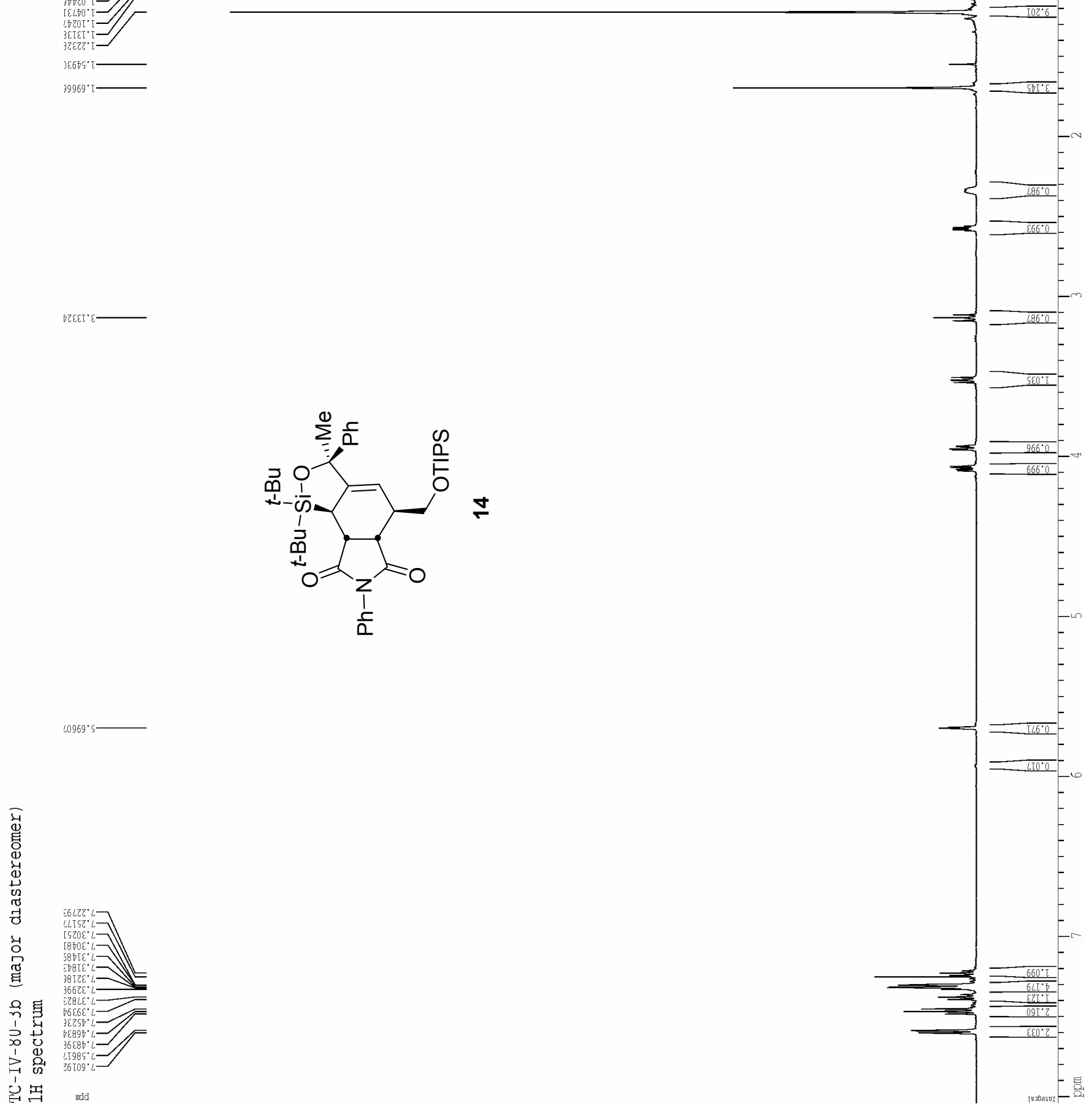


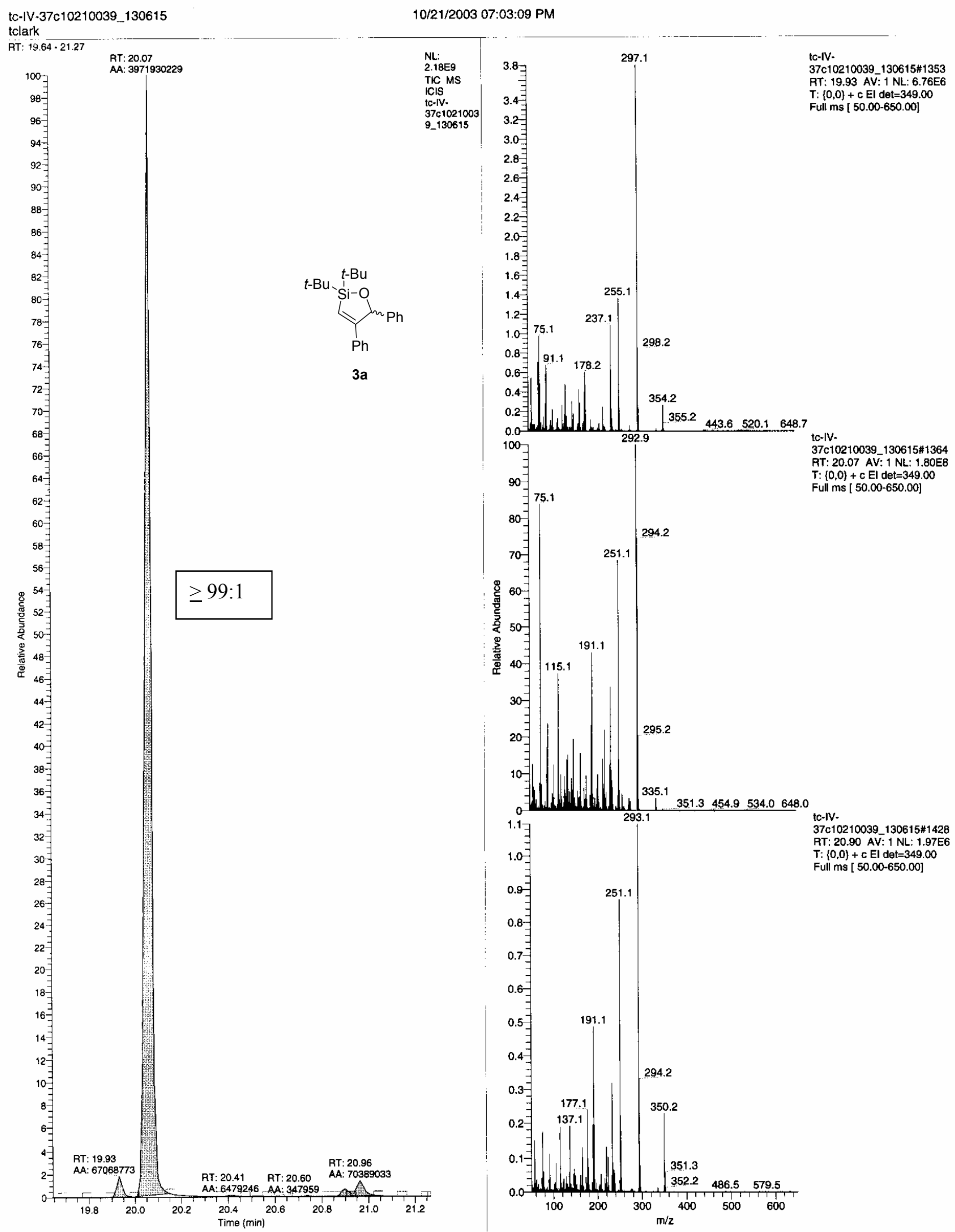




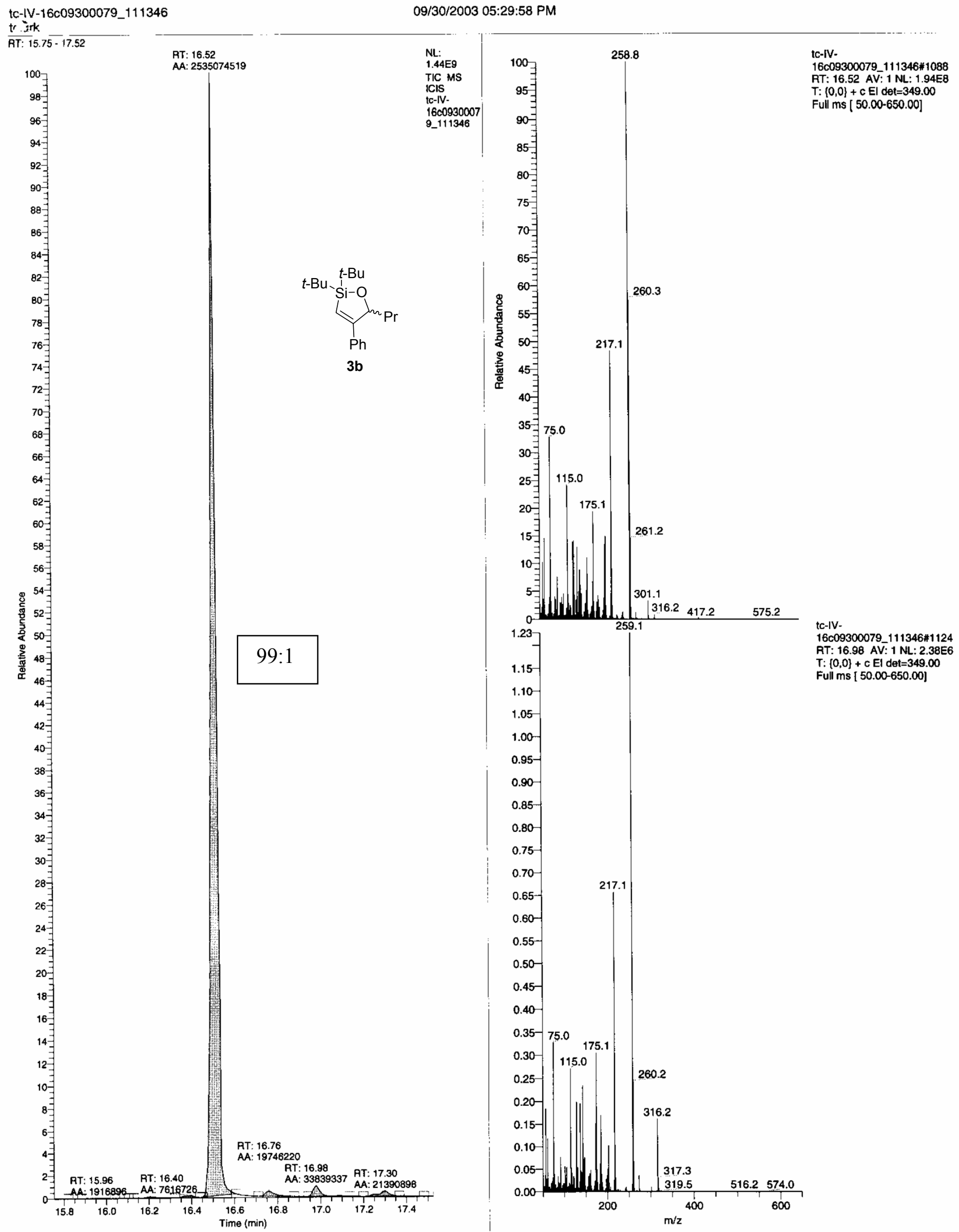




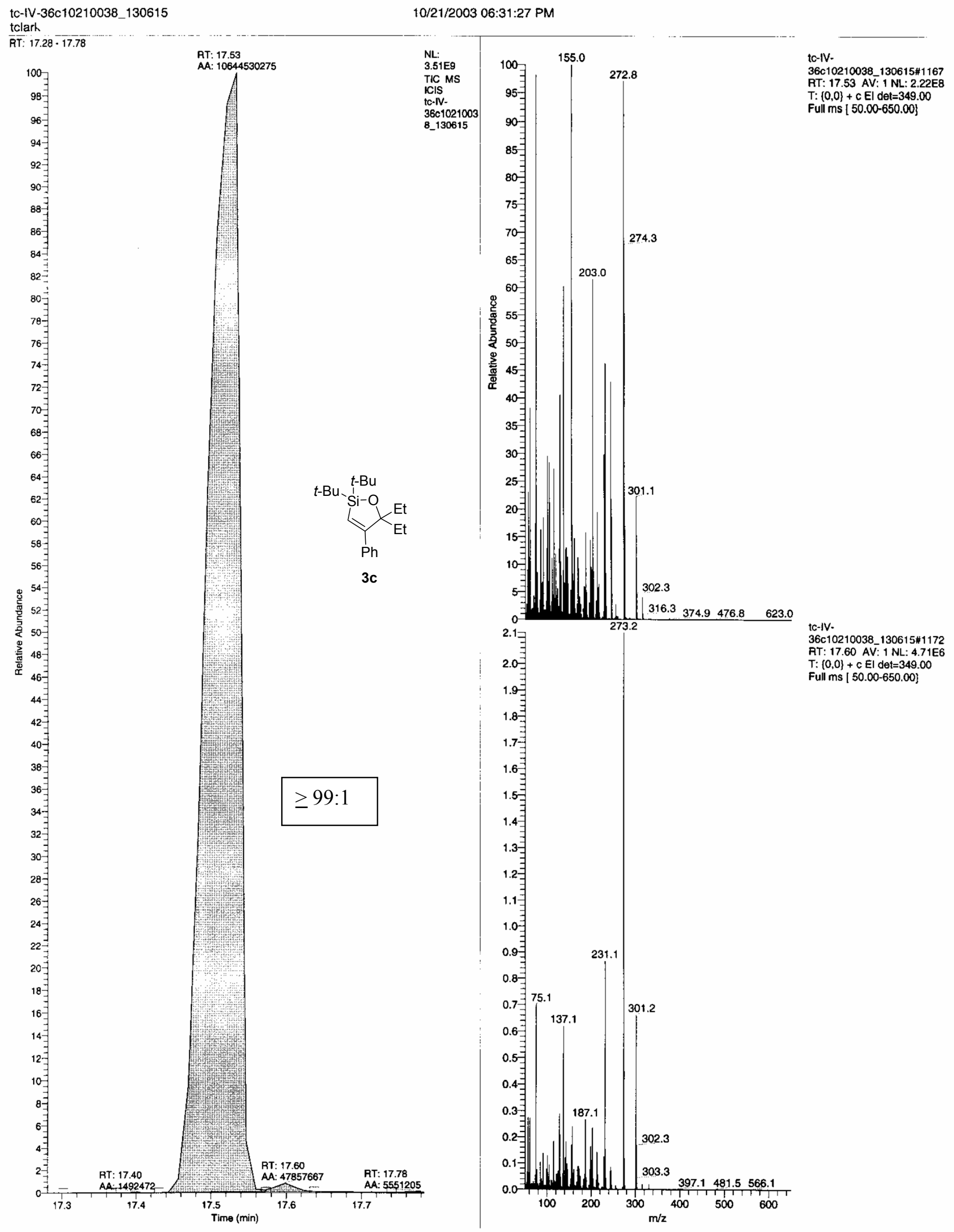




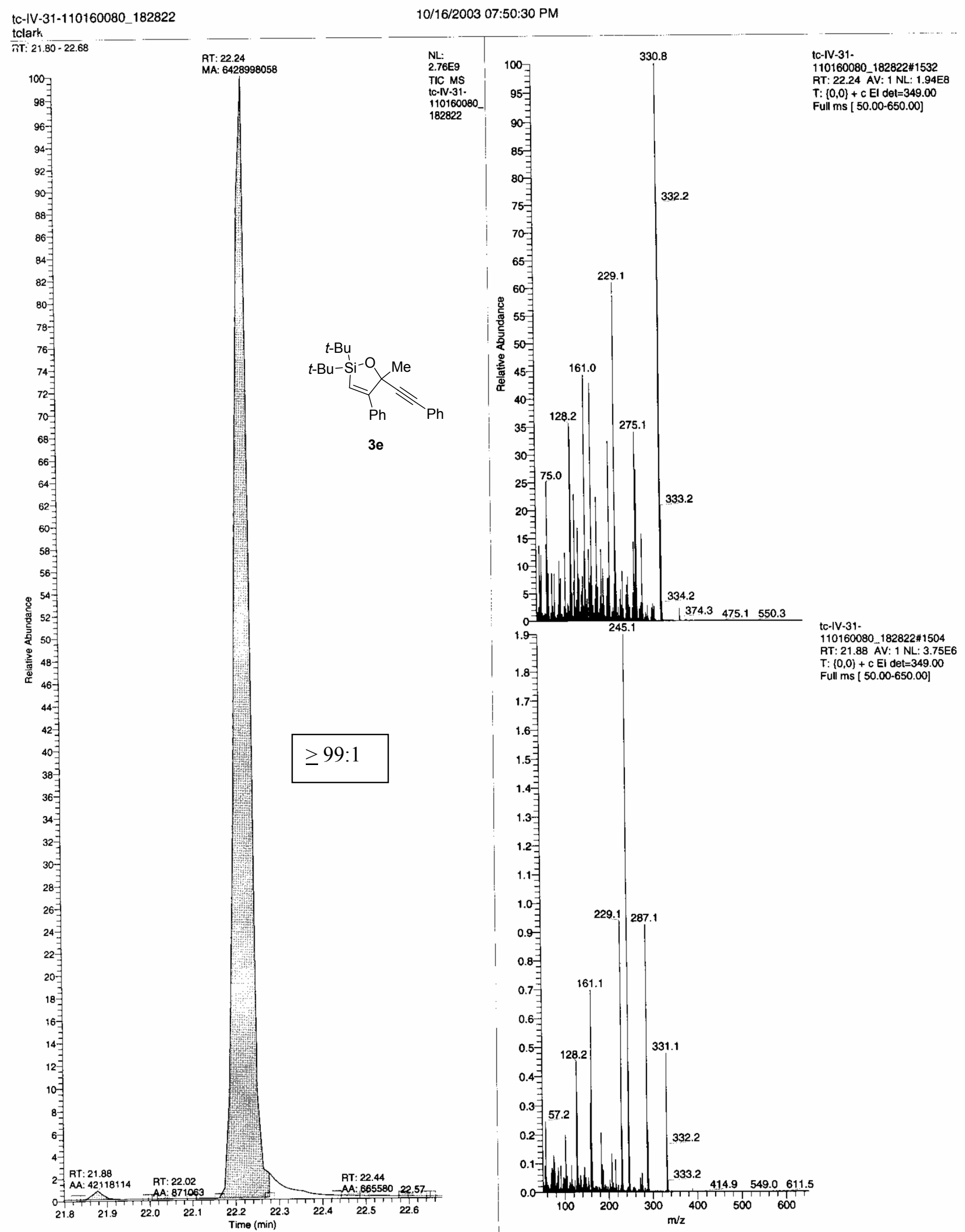




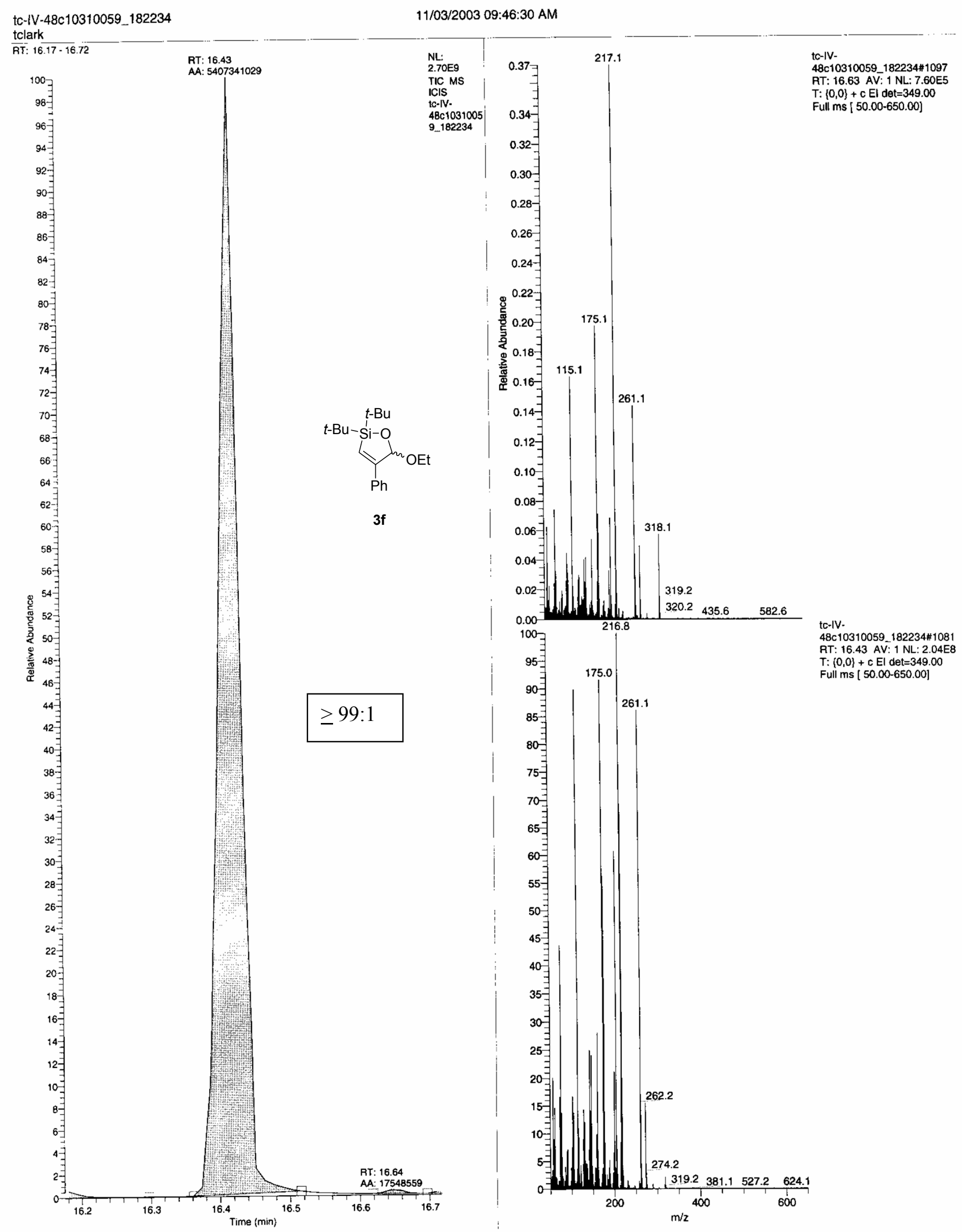




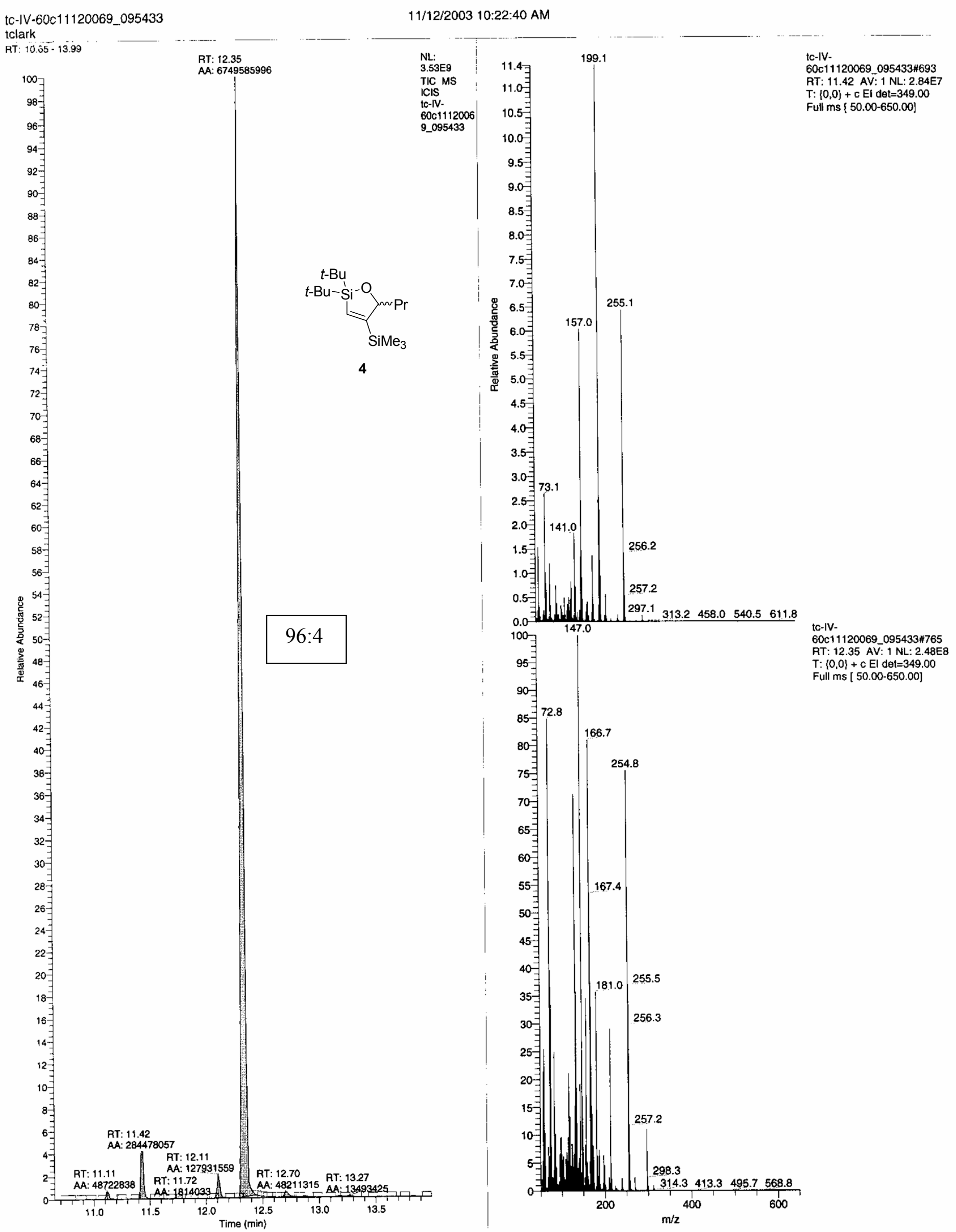


tc-IV-77c12080124_17542।

tclatk

RT: $22.82 \cdot 24.18$

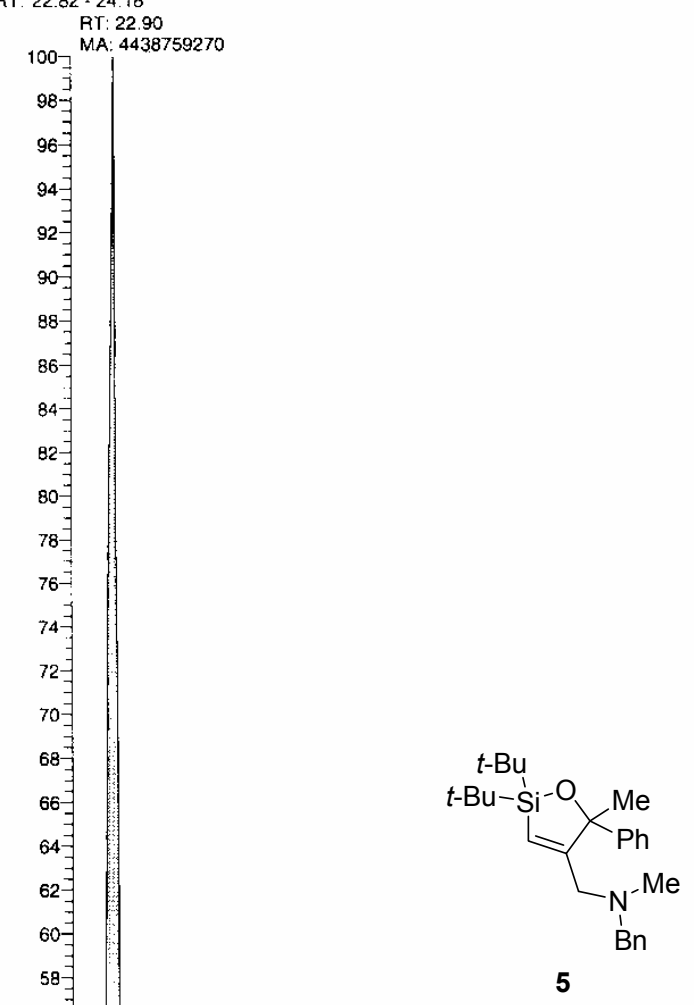

56

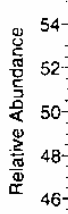

$90: 10$

AT: 23.68

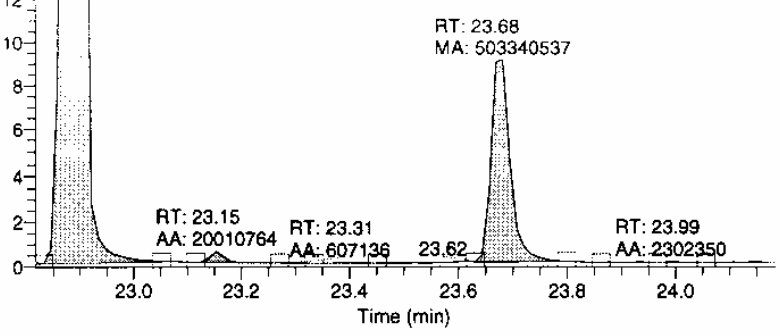

12/08/2003 07:13:00 PM

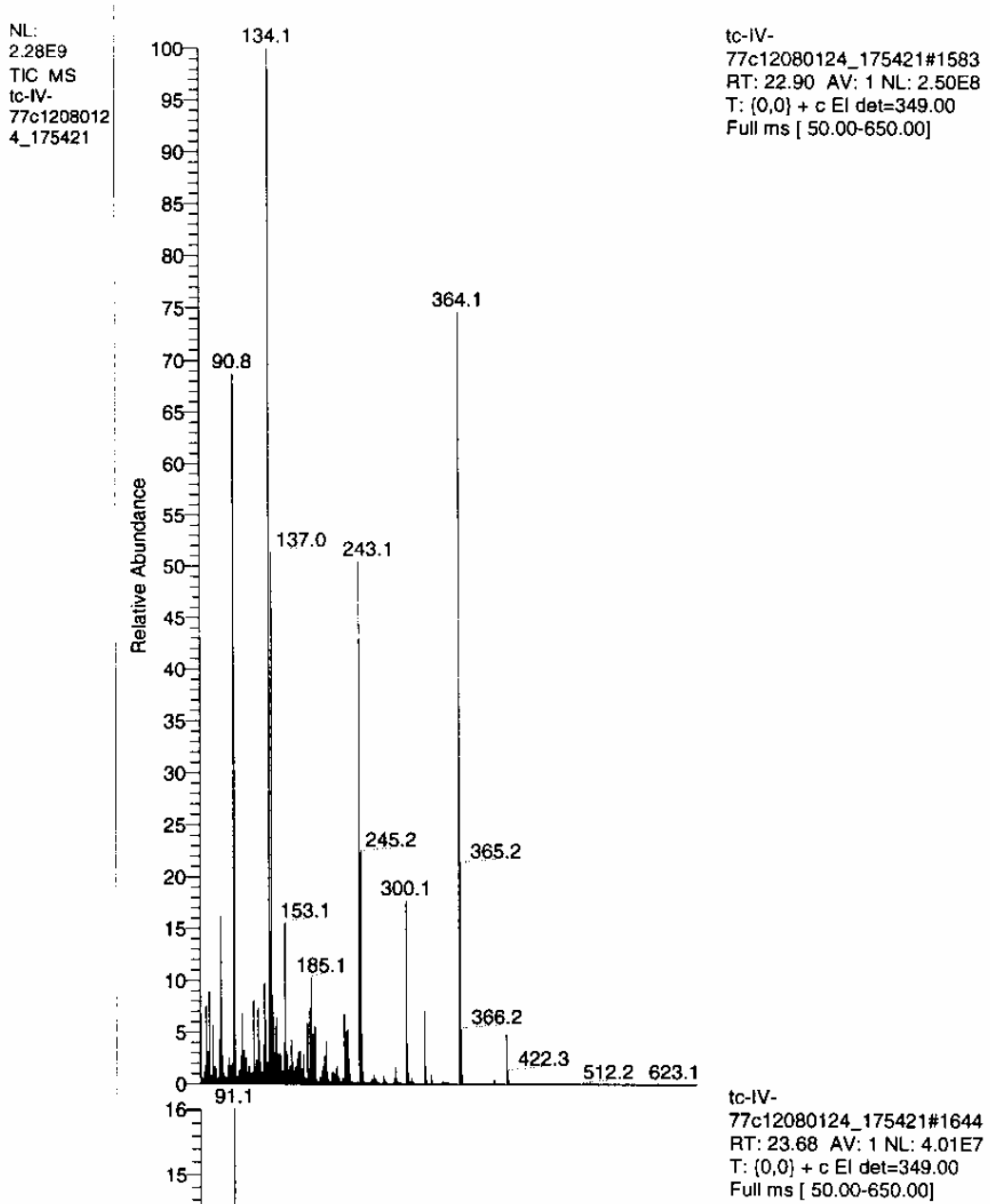




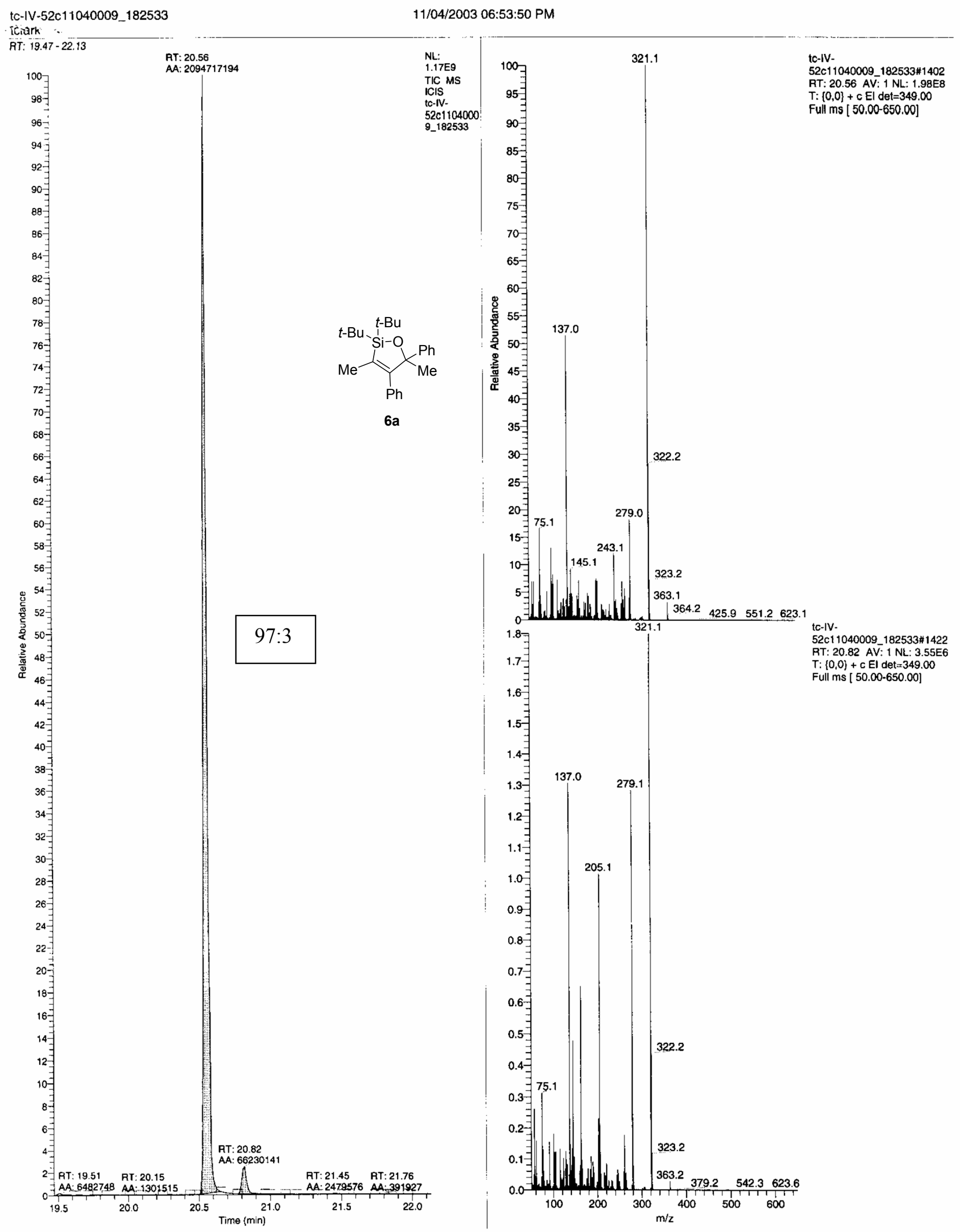




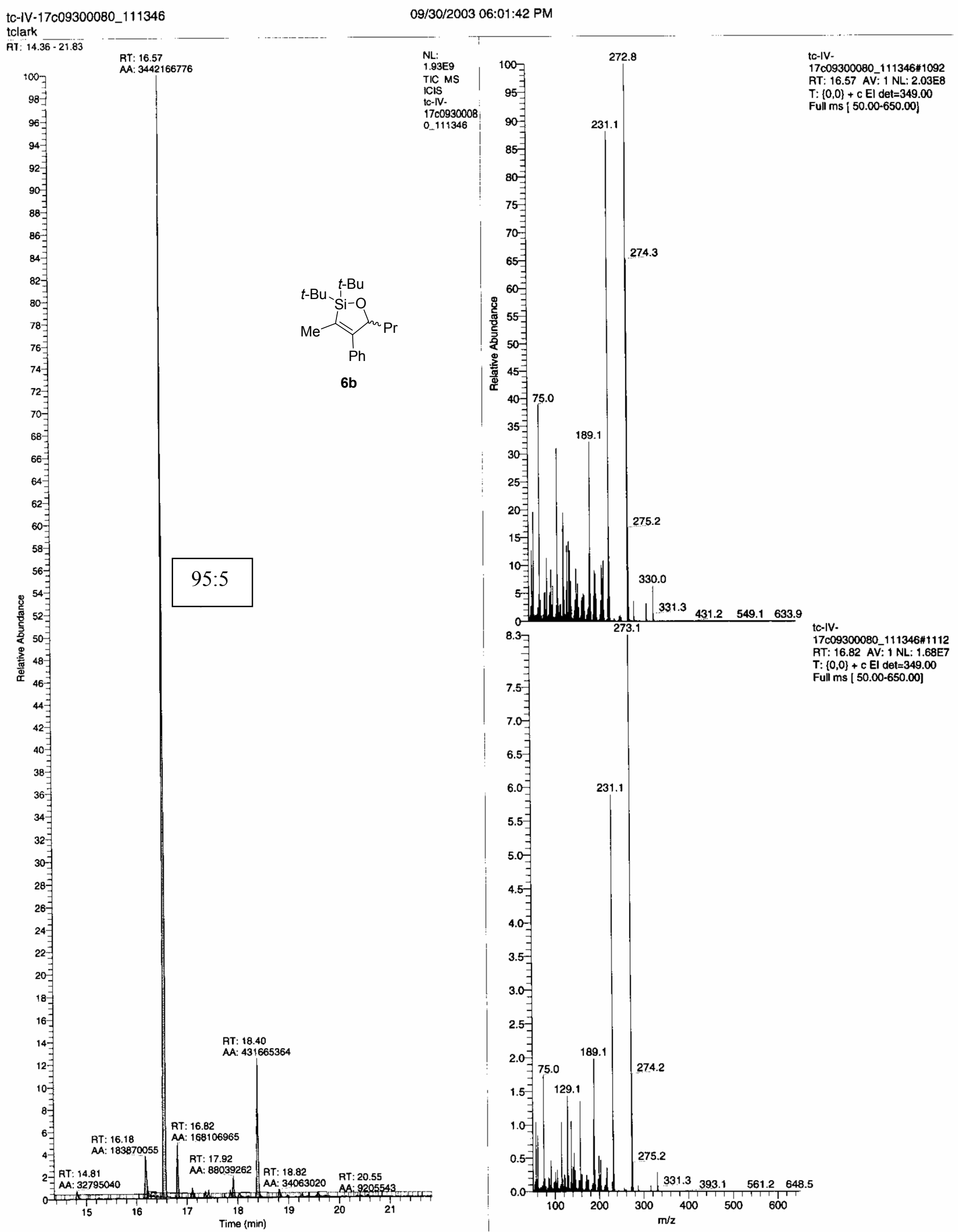




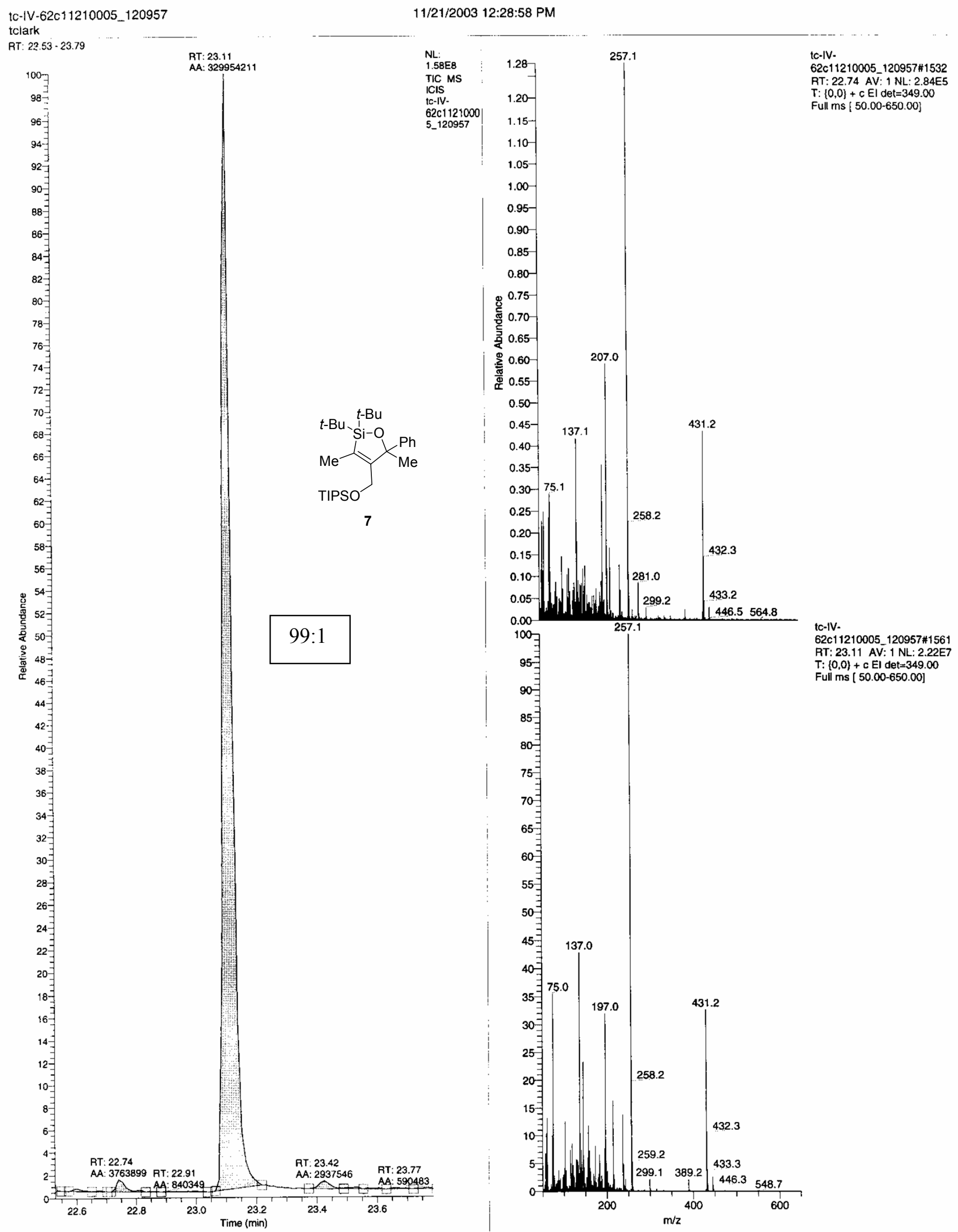




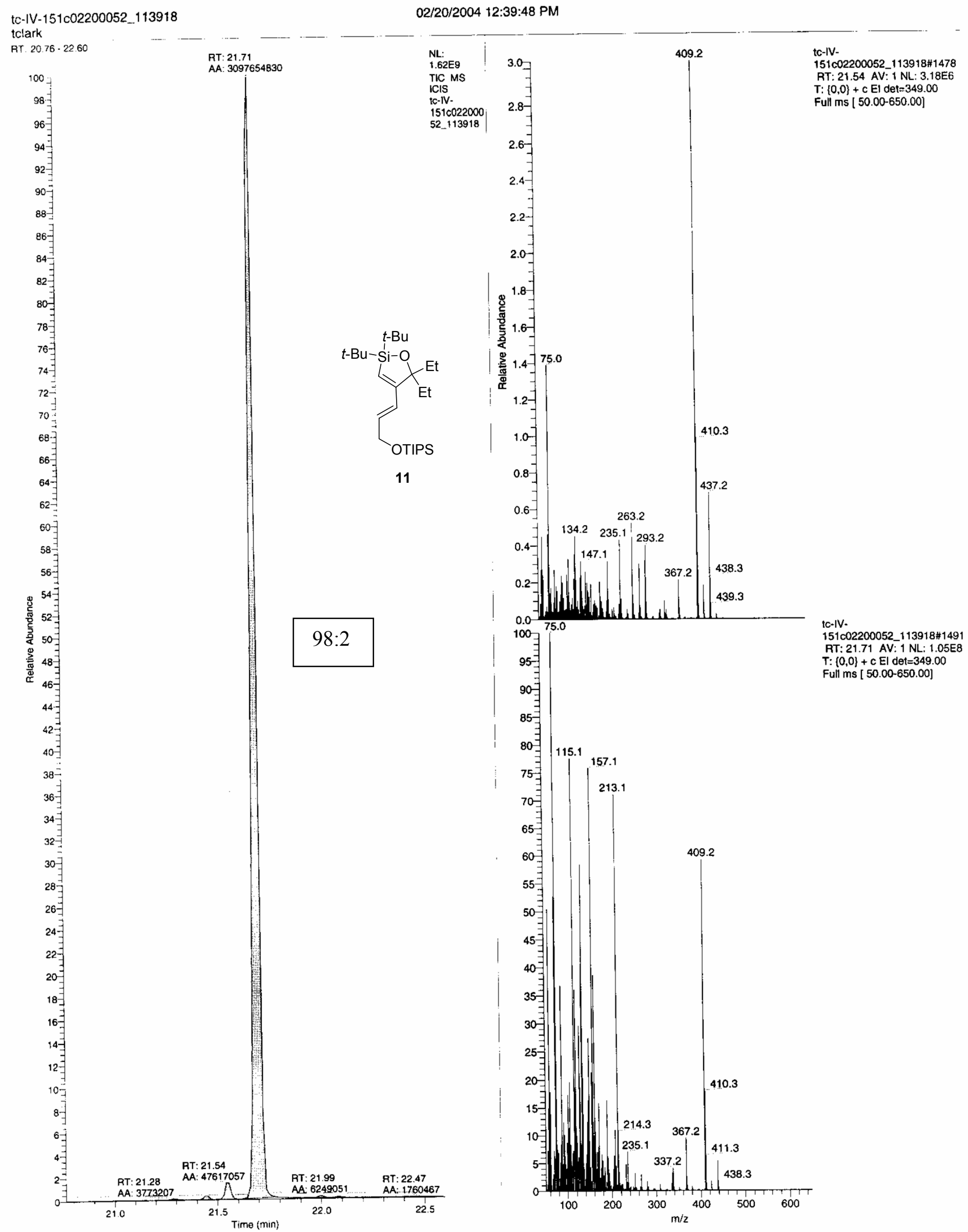




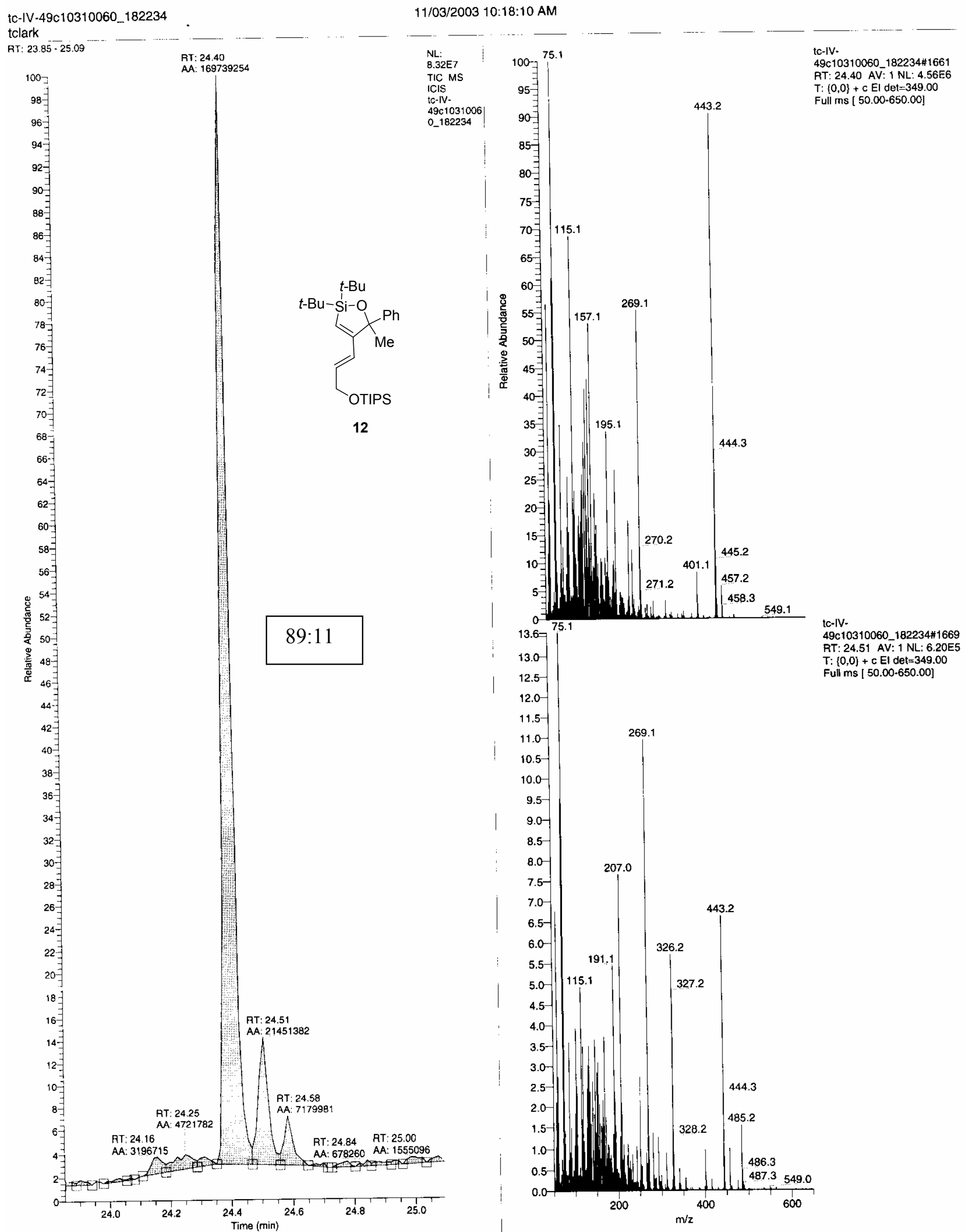




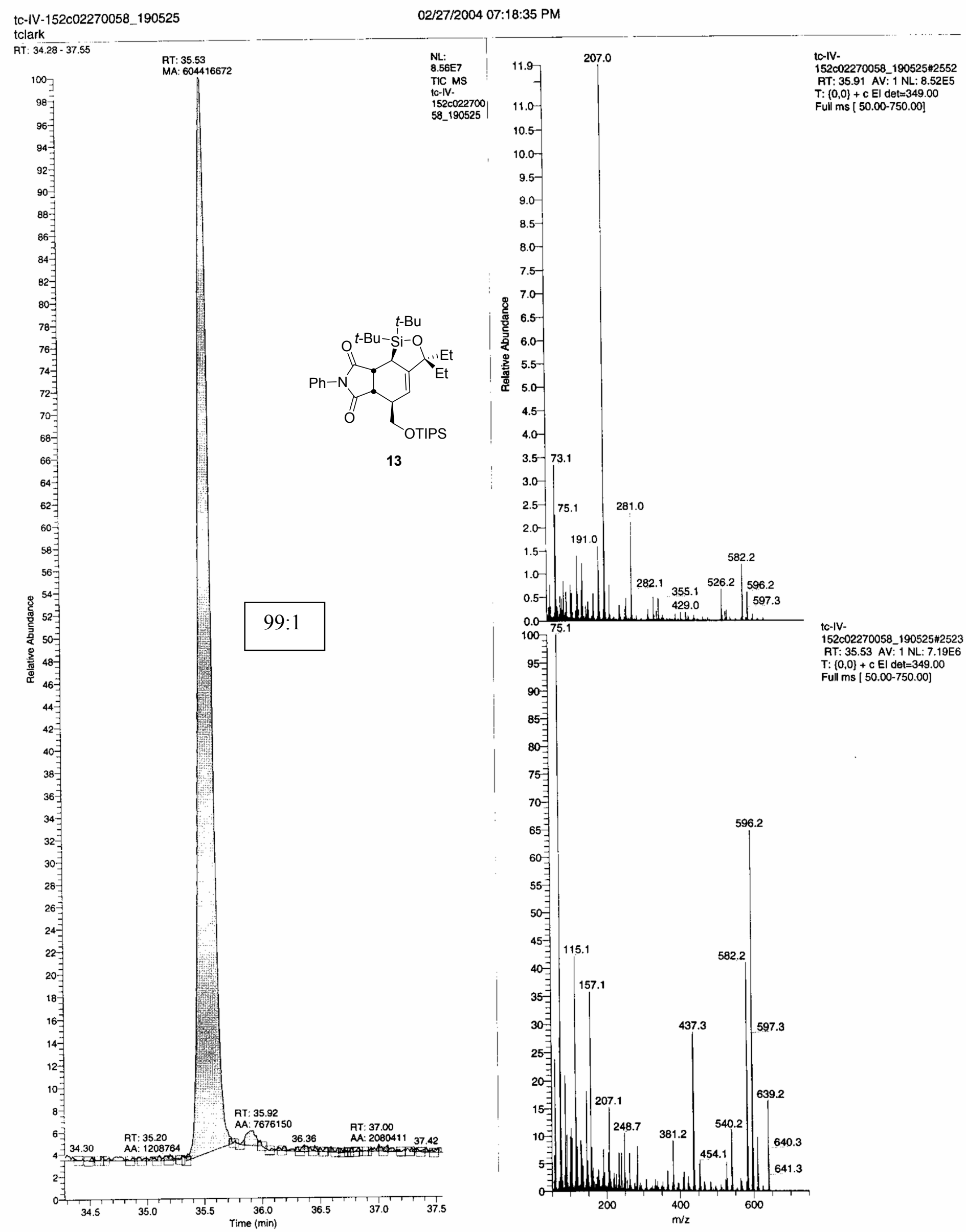


tc-IV-34c10250007 172332

tclark

RT: $16.15 * 18.36$

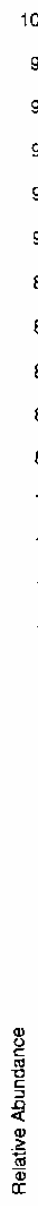

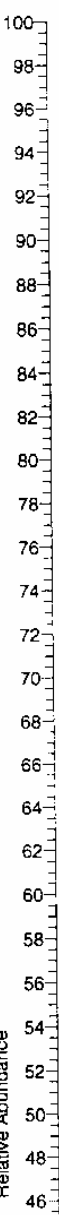

42

38

36

32

28

\section{(n)

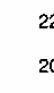

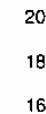

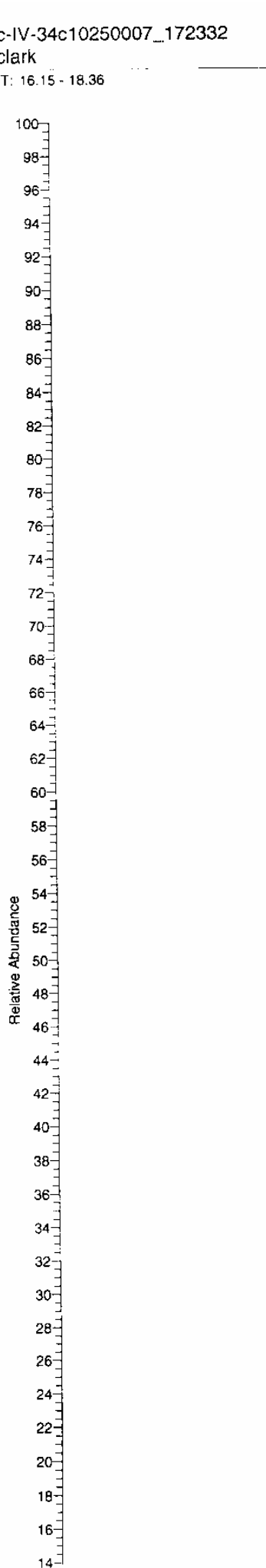

$$
\text { 萡 }
$$

